\title{
Polymer embedding for ultrathin slicing and optical nanoscopy of thick fluorescent samples
}

\author{
Dissertation \\ zur Erlangung des Doktorgrades der \\ Mathematisch-Naturwissenschaftlichen Fakultäten \\ der Georg-August-Universität zu Göttingen
}

vorgelegt von

Annedore Punge

aus Soest

Göttingen 2009 
D7

Referent: Prof. Dr. Jürgen Troe

Korreferent: Prof. Dr. Stefan W. Hell

Tag der mündlichen Prüfung: 


\section{Abstract}

Optical far-field methods are frequently recruited in modern life sciences, especially because one can benefit from various kinds of specific labeling. This was forced even more when several techniques for diffraction-unlimited imaging came up within the last two decades, the first of them STimulated Emission Depletion nanoscopy (STED). The lateral resolution refinement paved the way to a whole new era of addressable biological questions. Inevitably, the call for an enhanced axial resolution arose shortly after. Within this work, a straightforward approach for an entirely three-dimensional investigation of arbitrarily thick samples with nanoscopic resolution is presented. STED nanoscopy is combined with mechanical ultrathin slicing to a section thikness of $50-100 \mathrm{~nm}$ in the axial direction. This approach is highly advantageous, not only because ultrathin sectioning reveals densely packed structures with a, compared to a confocal spot size, tenfold increased resolution in the axial direction. Importantly, the fluorescence background from adjacent layers of the focal plane is avoided. Furthermore, every single layer of a three-dimensional sample can be addressed independently with the optical highresolution provided by STED. Hence, photo-bleaching in the periphery of the focal plane as well as scattering and aberrational effects within the sample are excluded. As a result, three-dimensional nanoscopic investigation is no longer limited to sophisticated experiments, including complex setups or computational deconvolution of the data. Even more important, it can be performed on arbitrarily thick samples, like whole tissue blocks. 



\section{Contents}

1 Motivation 1

\begin{tabular}{|lll}
2 & Theoretical Background & 3
\end{tabular}

$2.1 \quad$ Far-field optical microscopy $\ldots \ldots \ldots \ldots$. . . . . . . . . . . . . . 3

$2.1 .1 \quad$ Fluorescence microscopy $\ldots \ldots \ldots$. . . . . . . . . . . . . 4

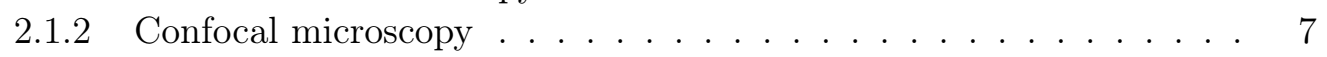

$2.2 \quad$ High resolution fluorescence microscopy $\ldots \ldots \ldots$. . . . . . . . . . 8

2.2 .1 The lateral direction $\ldots \ldots \ldots \ldots$

2.2 .2 The axial direction $\ldots \ldots \ldots \ldots$

2.3 The mechanical sectioning approach . . . . . . . . . . . . . . . . . . . . . 19

$2.3 .1 \quad$ Ultramicrotomy $\ldots \ldots \ldots \ldots$. . . . . . . . . . . . . . . . . . . . . . . 19

$2.3 .2 \quad$ Embedding polymers . . . . . . . . . . . . . . . . . . . . . 23

$\begin{array}{lll}3 & \text { Experiments and results } & 31\end{array}$

$3.1 \quad$ Embedding media for high-resolution light microscopy . . . . . . . . . . . 31

$3.1 .1 \quad$ Auto-fluorescence of the polymers . . . . . . . . . . . . . . 32

3.1 .2 Refractive indices of the polymers . . . . . . . . . . . . . . . . 34

3.1 .3 Properties of fluorescent dyes in polymers . . . . . . . . . . . . . 35

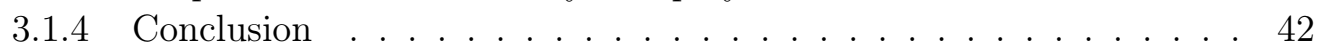

3.2 STED nanoscopy on ultrathin sections . . . . . . . . . . . . . . . 44

$3.2 .1 \quad$ Fluorescence inhibition on ultrathin sections . . . . . . . . . . 45

$3.2 .2 \quad$ STED imaging on ultrathin sections . . . . . . . . . . . . . . . 47

3.2.3 $\quad$ STED imaging of ultrathin sectioned vs. bulk structures . . . . . . 49

3.2.4 Ultrathin mechanical vs. optical sectioning (isoSTED) in STED nanoscopy $\ldots \ldots \ldots \ldots \ldots \ldots \ldots \ldots$

3.3 Application I: 3D Investigation of a cultured neuronal network . . . . . . 57

3.4 Application II: Nanoscopy on fluorescent fusion proteins in C. elegans . . 72

3.5 Application III: Nanoscopy on mammalian tissue $\ldots \ldots \ldots$. . . . . . . . 86

4 Conclusion and Outlook 97

$\begin{array}{lll}5 & \text { Appendix } & 101\end{array}$ 



\section{Abbreviations}

\begin{tabular}{|c|c|}
\hline $3 \mathrm{D}$ & $=$ three dimensional \\
\hline AFM & $=$ atomic force microscopy \\
\hline AFS & $=$ automatic freeze substitution \\
\hline $\mathrm{APD}$ & $=$ avalanche photo detector \\
\hline ATLUM & $=$ automated tape collection in lathe ultramicrotome \\
\hline ATUM & $=$ automated tape collection in ultramicrotome \\
\hline B52 & $=p$-toluene sulfonic acid \\
\hline BDMA & $=N, N$-dimethylbenzylamine \\
\hline BSA & $=$ bovine serum albumine \\
\hline CARS & $=$ coherent anti-stokes raman spectroscopy \\
\hline CLSM & $=$ confocal laser scanning microscope \\
\hline $\mathrm{CW}$ & $=$ continous wave irradiation \\
\hline D-Rex & $=$ dark state relaxation \\
\hline DABCO & $=[1,4]$-diazabicyclo- $[2.2 .2]$-octane \\
\hline DAPI & $=4^{\prime}, 6$-diamidino-2-phenylindole \\
\hline DBP & $=$ dibutyl phtalate \\
\hline DDSA & $=$ dodecenyl-succinic-anhydride \\
\hline DER736 & $=$ polymer of epichlorohydrin-polyglycol \\
\hline DIC & $=$ differential interference contrast \\
\hline DMA & $=N, N$-dimethylaniline \\
\hline DMP-30 & $=$ tris-dimethylaminomethyl phenol \\
\hline DMSO & $=$ dimethyl sulfoxide \\
\hline DNA & $=$ desoxy-ribonuclein-acid \\
\hline
\end{tabular}




$$
\begin{array}{ll}
\text { DNC } & =\text { dorsal nerve cord (of C. elegans) } \\
\text { EELS } & =\text { electron energy loss spectroscopy } \\
\text { EM } & =\text { electron microscopy } \\
\text { FCS } & =\text { fluorescence correlation spectroscopy } \\
\text { FP } & =\text { fluorescent protein } \\
\text { FWHM } & =\text { full width at half maximum } \\
\text { GA } & =\text { glutaraldehyde } \\
\text { GFP } & =\text { green fluorescent protein } \\
\text { GSD } & =\text { ground state depletion } \\
\text { GSDIM } & =\text { ground state depletion with individual molecule return } \\
\text { HMME } & =\text { hexamethylolmelamine-methyl ether } \\
\text { HPF } & =\text { high pressure freezing } \\
\text { IC } & =\text { internal conversion } \\
\text { ISC } & =\text { intersystem crossing } \\
\text { MEA } & =\text { mercaptoethylamine } \\
\text { MME7002 } & =\text { hexamethylolmelamine-methyl ether, } 70 \% \text { solution in water } \\
\text { NMA } & =\text { methylnadic anhydride } \\
\text { NMJ } & =\text { neuro-muscular junction } \\
\text { NSOM } & =\text { near field scanning optical microscopy } \\
\text { PV } & =\text { PALM }
\end{array}
$$




$$
\begin{aligned}
& \text { PVA }=\text { polyvinyl alcohol } \\
& \text { Qeff } \quad=\text { quantum efficiency } \\
& \text { RESOLFT }=\text { reversible saturable optical fluorescence transition } \\
& \mathrm{S} \quad=\text { singlet state } \\
& \text { SDS } \quad=\text { sodium dodecyl sulfate } \\
& \text { SLM }=\text { spatial light modulator } \\
& \text { SMI }=\text { spatially modulated illumination } \\
& \text { SNAP }=\text { soluble } N \text {-ethylmaleimide-sensitive-factor attachment protein } \\
& \text { SNARE }=\text { soluble } N \text {-ethylmaleimide-sensitive-factor attachment receptor } \\
& \mathrm{SPDM}=\text { spectral position determination microscopy } \\
& \text { SPEM = saturated patterned excitation microscopy } \\
& \mathrm{SR}=\text { serine/arginine-rich } \\
& \text { SSIM = saturated structured illumiination microscopy } \\
& \text { STED }=\text { stimulated emission depletion } \\
& \text { STORM = stochastic optical reconstruction microscopy } \\
& \mathrm{T} \quad=\text { triplet state } \\
& \text { T-Rex = triplet relaxation } \\
& \mathrm{TDE}=2,2^{\prime} \text {-thiodiethanol } \\
& \text { TEM = transmission electron microscopy } \\
& \text { TIRF }=\text { total internal reflection fluorescence } \\
& \mathrm{UV}=\text { ultra violet } \\
& \text { VCD = 4-vinyl-1-cyclohexene-diepoxide } \\
& \mathrm{VNC}=\text { ventral nerve cord (of C. elegans) } \\
& \varepsilon \quad=\text { molar extinction coefficient } \\
& \lambda \quad=\text { wavelength } \\
& \eta \quad=\text { depletion factor } \\
& \tau_{f l} \quad=\text { fluorescence lifetime }
\end{aligned}
$$





\section{Motivation}

The Anglo-Saxon saying "seeing is believing" justifies why imaging is one of the most employed methods in modern science. Clearly, one should always bear in mind that due to the human fallibility, the reverse might be true. However, theoretical assumptions are generally easier established if there are means of visually confirming the predictions. Unfortunately, while looking at small objects, the naked human eye is restricted to a resolution of $0.1 \mathrm{~mm}$, meaning that closer features cannot be distinguished anymore [22]. Thus, tiny objects are difficult or even impossible to investigate in terms of their size, shape, structure or material. The same applies to herewith connected natural processes.

Already the Greek and Romans realized that natural phenomena can be followed through a lens, sometimes just a water-droplet, which focuses the light and helps to make small details visible. However, it took up to the early 17 th century until the first microscopes were built by combining several lenses in a row. They facilitated the challenging observation of bacteria, muscle fibers, blood flow or single cell organisms [44]. In the beginning of the 19th century, another significant step forward was made when Ernst Abbe, in close collaboration with Carl Zeiss, investigated the wave-character of light [2]. Consequently the microscopic resolution was improved by a factor of two. Abbe also derived his famous resolution formula for optical microscopes:

$$
\Delta x, \Delta y \simeq 1.22 \frac{\lambda}{N A}, \Delta z \simeq 4 \frac{n \lambda}{N A^{2}} .
$$

Here, $\lambda$ is the wavelength of light and $N A=n \cdot \sin \alpha$ the numerical aperture of the lens. With the best objective lenses used in modern light microscopy, a semi-aperture angle $\alpha$ of $73^{\circ}$ can be achieved leading to a highest lateral resolution of $150 \mathrm{~nm}$ (and $N A=1.6$ ), assuming that UV light $(350 \mathrm{~nm})$ is used. For the axial direction, the resolution of a diffraction-limited system is around $3-4$ times worse.

Taking into account that many natural processes occur on the molecular level, it is evident that such a resolution is very often insufficient. According to eqn. 1.1, higher resolution is achievable by either reducing the observation wavelength or by increasing the numerical aperture. The first led to the establishment of electron microscopy, which can under ideal conditions resolve structures down to the size of an Angstrom. It is not even limited by the wavelength of the electrons, but rather by aberration effects [185]. In contrast, in near-field methods [136] like atomic force microscopy (AFM) [128, 45] or scanning near-field optical microscopy (SNOM) [102, 164, 98, focusing of light is needless and restrictions by the numerical aperture are eliminated. To collect the measuring signal, a sensor tip is closely approached to the sample surface. Consequently only the exterior of a sample is accessible by those techniques, while any interior remains concealed to the observer. 


\section{Motivation}

Still, for the biological investigation of thick cellular structures very often light microscopic resolution on the nanoscale is indispensable or at least desirable, be it due to labeling issues or due to the incompatible working conditions of other high-resolution methods. Starting from the early 1990s new optical high-resolution methods were conceived in the fields of fluorescence microscopy. These evolved out of a revised notion of the imaging process, which explicitly included the photophysical properties of the fluorescent dyes and exploited finely tuned fluorescence switching mechanisms. The most common concepts are STED [69, 103, PALM [11, 75] and STORM [144], which were all shown to provide a lateral resolution of $20 \mathrm{~nm}$ or less with focused light. However, attaining resolution below $100 \mathrm{~nm}$ also in the axial direction still significantly increases the experimental complexity or at least requires extraordinary stable and bright dyes [30, 86, 147, 154]. What is even more critical is the limited penetration depth of a focused light beam into a sample, typically in the range of a few micrometers, because of scattering effects in the interior. This makes spatially extended samples only partly accessible by optical high resolution techniques. Furthermore, very dense structures often cause a background signal, which can compromise the high-resolution images. Tackling these limitations is the aim of this work.

Some of the listed problems are not new, but arose around 60 years ago for transmission electron microscopy (TEM) [101]. Mechanical ultrathin sectioning of the sample had in many applications a pivotal role and vitally contributed to the success of the technique. Hence, it is logical to adopt this strategy for optical methods, including STED microscopy, as well. However, for the preparation of slices which are a few tens of nanometers thin, previous embedding into a hard plastic block is normally mandatory and accepted.

However, polymer embedding is not only incompatible with life-cell imaging. There are also substantial changes in the direct environment of the sample. If the samples are prepared as usual and stained before embedding, the effect is most notably at the site of the fluorescent labels. The fluorophores are potentially either destroyed by the chemical polymerization reaction or their spectral properties vary with the surroundings.

In contrast, staining the samples after polymer embedding will probably result in an inefficient staining. The polymerization and the subsequent slicing process are likely to affect the labeling sites, making them hard to recognize by the labeling targets. In addition, distractive staining artifacts occur more often in the unpolar polymer environment. Hence, post-embedding labeling is a rather unattractive alternative.

Finding ways to sustain the benefits of optical microscopy, in particular in terms of specific labeling, within high-resolution methods during polymer embedding is the major goal of this work. To this end, the spectral changes of fluorophores in a polymeric environment are investigated.

In addition, different polymers show a different applicability to fluorescence microscopy methods, especially when high laser power is employed. Therefore, their optical properties will be addressed and balanced out.

Finally, the practical use of the method is verified through tackling different biological questions. All of them vitally benefit form the nanoscopic resolution in all three spatial dimensions, enabled through the combination of the most powerful optical and mechanical tools. 


\section{Theoretical Background}

\subsection{Far-field optical microscopy}

Due to the wave character of electromagnetic radiation, the resolution capability of an optical far-field system is restricted by diffraction [2]. A point-shaped object, which is imaged with a light microscope, is not reproduced as a simple point, but smeared out in all three dimensions. The resulting diffraction pattern for a circular aperture with its alternating maxima and minima, also called Airy pattern, is mathematically described as

$$
I(\varphi)=I_{0}\left[\frac{J_{1}(\Phi / 2)}{(\Phi / 4)}\right]
$$

with $\Phi=\frac{2 \pi B \sin \varphi}{\lambda}$. Here $I$ describes the intensity subject to the observation angle $\varphi, B$ is the diameter of the pinhole, $\lambda$ the wavelength of the incoming light and $J_{1}$ the 1st order Bessel function. The central area of the pattern is called Airy disc and is the smallest area to which one can focus a beam of light through an optical system.

Considering that the function determining how an object is imaged is called pointspread-function $h(\vec{r})$ (PSF) and in the case of a linear, spatial invariant optical system, the imaging process can be described as a convolution of the object distribution $O(\vec{r})$ with the PSF. This results in the image distribution

$$
B(\vec{r})=O(\vec{r}) \otimes h(\vec{r})
$$

The effect of the PSF on imaging is illustrated in Fig.2.1. In a diffraction limited optical system the PSF is closely correlated with its resolution capability. According

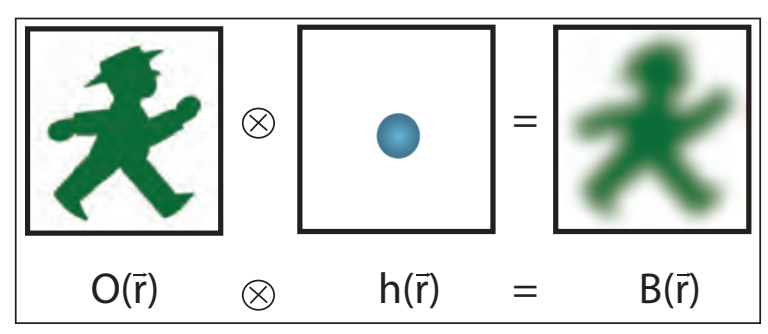

Figure 2.1: Visualization of the blurring of an object during image acquisition with a PSF of a certain size. Mathematically this effect is described by convolving the object distribution $O(\vec{r})$ with the PSF $h(\vec{r})$ resulting in the blurred image distribution $B(\vec{r})$. 


\section{Theoretical Background}

to the Rayleigh limit it can be roughly assumed that two points with a distance of the full-width-half-maximum (FWHM) of the PSF -which is equal to the lateral distance between the minima of $h(\vec{r})$ that enclose the main maximum or half the diameter of the Airy disk- can be barely resolved with the system.

Thus it becomes evident that the resolution of an optical system is limited, and if two structures get too close, the system-induced images of those structures overlap. The consequence is that they cannot be distinguished anymore and no precise conclusion about the number of objects or their in between distance can be made [15].

\subsubsection{Fluorescence microscopy}

With increasing complexity of a sample, a good imaging contrast is of importance to resolve single and small structures. Subsequent to the observation of luminescent samples after irradiation with UV light, August Köhler invented the concept of fluorescence microscopy in 1908 [131. This enabled a selective observation of a certain structure after staining it with appropriate labels. In consequence, the detection sensitivity was remarkably increased.

The principle of fluorescence can be explained by considering a simple system with two distinct energy-levels as described by Einstein, where absorption, spontaneous and stimulated emission occur [32]. The effect is commonly explained by means of the Jablonski diagram shown in Fig.2.2. A fluorescence molecule is excited by light of a certain wavelength $\lambda_{\text {exc }}$ from its electronical ground state $S_{0}$ to an excited state of higher energy, e.g. $S_{1}^{\text {vib }}$. From this electronical and vibrational excited state it relaxes very fast to the vibrational ground state $S_{1}$ (Kasha's rule) and afterwards under emission of a -with regard to the excitation normally red-shifted- photon of the wavelength $\lambda_{\mathrm{f}}$ to a vibrational excited electronical ground state $S_{0}^{v i b}$. While the molecule is in its $S_{1}$ state, an electronic transition to the ground state may not only occur due to spontaneous fluorescence emission, but it can be stimulated by an incident photon, which triggers the emission of a second coherent photon from the molecule. Once the molecule is back in its vibronic ground state, it is ready for a new excitation-emission cycle. When the emitted fluorescence photons are detected, the (blue-shifted) excitation light is usually blocked by adequate filters. In many cases the photophysics of a fluorescent molecule within a (biological) environment are not that simple and a lot of additional processes like triplet-formation or destructive photo-bleaching might occur (see Fig.2.2. A). Nevertheless a huge number of biological and other questions has been successfully tackled by labeling most different structures with chromophores and observing them under a fluorescence microscope. Additionally worth mentioning is that a change in the photophysical behavior of the chromophore as a consequence of environmental changes might even be a source for additional local information [166].

The decision which fluorescent dye is to be used in an application depends on many factors. First of all, an efficient excitation that results in a bright fluorescence signal and a high signal-to-noise ratio (SNR) is inevitable. This can be described by the effective quantum yield 


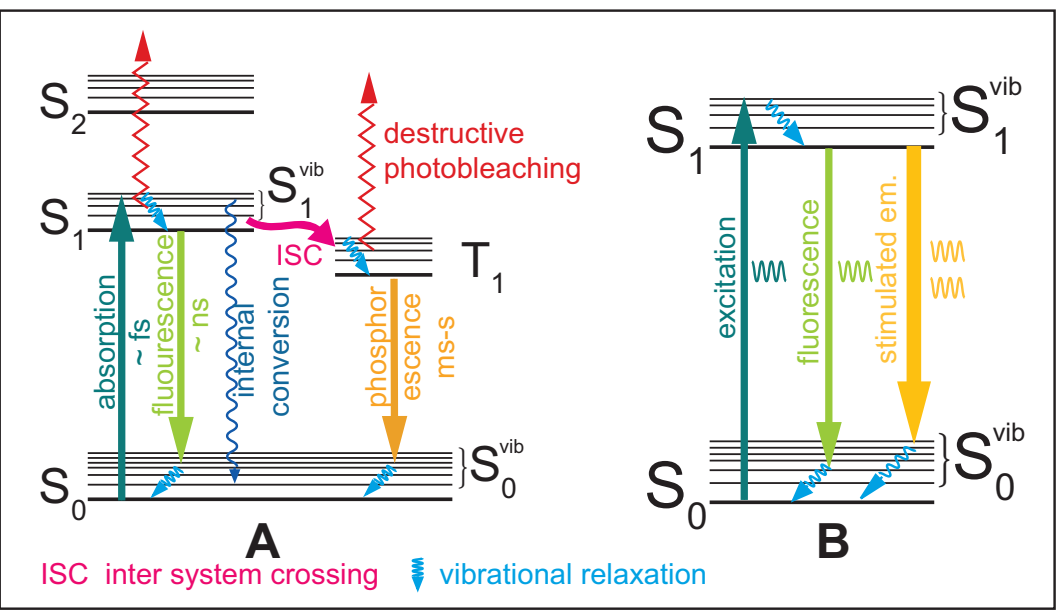

Figure 2.2: Jablonski diagram (left) and enlarged part of a Jablonski diagram to visualize the relevant processes for STED microscopy (right).

$$
Q_{\text {eff }}=\frac{n(\text { photon })_{\mathrm{emitted}}}{n(\text { photon })_{\mathrm{absorbed}}} .
$$

The number of photons absorbed by a certain fluorophore can be quantified through the molecular extinction coefficient $\varepsilon$ of it's solution.

$$
\varepsilon=\frac{E}{d} .
$$

$E$ describes the observed extinction and $d$ stands for the thickness of the transilluminated sample.

The number of emitted photons, however, might be reduced by a high tendency to perform intersystem-crossing and thereby triplet formation instead of fluorescence emission. Additionally, destructive photobleaching from an excited state or the presence of a fluorescence quencher might decrease the effective fluorescence quantum yield.

Furthermore, for fluorescence microscopy the absorption- and emission-profiles, including their shape or narrowness, and the energy-gap between them (Stokes-shift) are essential. The selection of a chromophore is dependent on the optical setup of the microscope. Mainly two issues are important: The excitation source should ideally fit the excitation maximum and the fluorescence maximum should be within the detection range, which is determined by the appropriate fluorescence filters.

Finally, the fluorescence lifetime $\tau$ of a chromophore is important. It refers to the average time a molecule stays in its excited state before emitting a photon. Typically the fluorescence follows first-order kinetics and the according exponential decay of the fluorescence intensity is described by

$$
I(t)=I_{0} \exp \left(-\frac{t}{\tau}\right) .
$$




\section{Theoretical Background}

For organic fluorescence markers in solution the value is typically a few nanoseconds.

If the chromophore is attached to biological samples, its physical properties like solubility in water or size (especially for proteins) can be of major issues as well.

Polymer embedding might have a considerable influence on any of those properties. It can be assumed that the number of deexciting collisions with freely diffusing molecules is substantially reduced as compared to a solution. This might increase both the effective quantum yield and the fluorescence lifetime. Moreover, compared to a solution, the number of freely diffusing oxidizing agents is reduced after polymer embedding. These oxidizers are very often responsible for the observed photobleaching after excitation of the fluorophore.

However, in the worst case, the polymer absorbs either the excitation light or the emitted fluorescence. Both imply a decreased effective quantum yield of the system.

In addition, polymer embedding might have a remarkable influence on the absorption or emission profile of a chromophore and cause spectral shifts. This has to be considered in microscopy, since it might require the adaptions of the optical system including the excitation wavelength or applied filters. For complex and therefore less flexible setups, it might even mean that a well established fluorophore is not viable anymore after embedding in a polymer. In STED microscopy in particular, it is important that the Stokes-shift does not becomes too small. This would cause a strenghtened reexcitation of the fluorophore by the STED beam.

Although more and more interesting labels are available, the most important chromophore classes for microscopy use are quantum dots, fluorescent proteins and organic fluorophores. Quantum dots, although bright and spectrally tunable, have the disadvantage to be toxic to biological tissue and show a high blinking behavior which makes them not very popular in life sciences. However, first attempts to overcome the blinking issue show the recent progress in this field, which might imply a rethinking in this context [174].

In the last 20 years fluorescent proteins have gained importance, especially for life cell imaging and in case of lacking specific membrane-permeable protein markers. But these still suffer from high bleaching rates and their sensitivity to environmental changes. This includes polymer embedding as well since in addition to potentially destructive chemical reactions, it requires a dehydration of the sample. Normally, a certain amount of crystal water is mandatory to make the proteins fluorescent.

Organic fluorophores are by far the most common labels. According to their chemical matrix they can be grouped into different classes. Essentially, these are rhodamines, cyanines, coumarines and fluoresceines, but the number of new, less prominent classes is steadily increasing. By synthetic variation of the sidechains, their spectral properties, their fluorescence behavior and also their solubility can be tuned to a large extent.

Of particular interest for high-resolution light methods are photoswitchable dyes, among them cyanine dyes (used in STORM [144], but restricted to membrane staining) and rhodamine dyes (applied for PALM and related methods [75, 36]).

However, to become a useful fluorescence dye, a chromophore has to possess the capability to work as a selective label. In selective biological studies proteins are the most frequently tackled structures. This is commonly realized by immuno-staining. The fluo- 


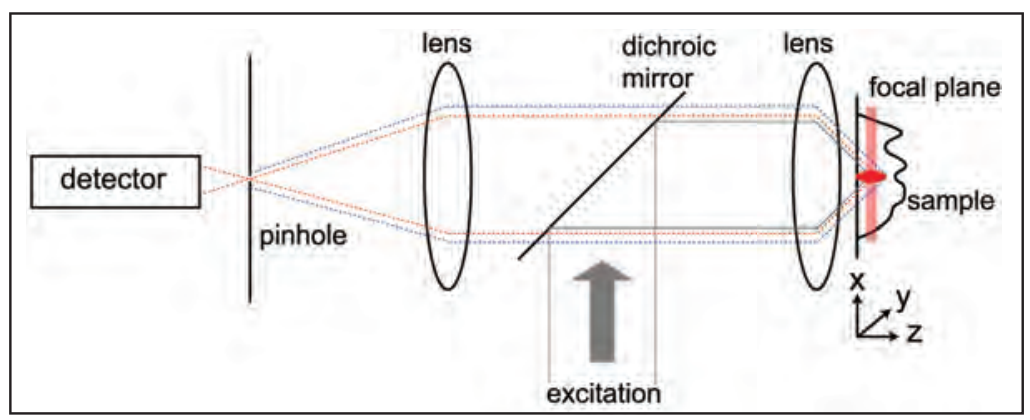

Figure 2.3: Setup and light paths of a confocal microscope.

rescent dye is directly attached to a primary antibody, which recognizes a specific protein (direct immuno-staining). A brighter staining can be achieved by using an unlabeled primary antibody, which is afterwards detected by several secondary antibodies, each of them carrying several molecules of the fluorescent dye (indirect immuno-staining).

Another straight-forward way to selective staining is achieved by fluorescent proteins (FPs). The cell is genetically forced to express a fusion protein consisting of the protein to be observed together with the fluorescent protein. However, both immuno-staining and genetically encoding, are using rather large labels that add an uncertainty to the structure (up to $20 \mathrm{~nm}$ ).

An overview about more sophisticated labeling strategies for high-resolution fluorescence methods, which play no essential role in this work is given elsewhere [35].

Conclusively, it has to be remarked that methods which are based on unspecific, e.g. hydrophobic interactions, are not suitable for polymer embedding. These interactions are likely to be effected by the embedding process, which then causes not intuitively assessable defects of labeling.

\subsubsection{Confocal microscopy}

Confocal microscopy is currently the most common concept to increase the axial resolution of an optical system. It is THE method for present day optical sectioning.

As introduced by Minski [126], in a confocal microscope the excitation and detection foci lay on top of each other or are "confocal". The point-like PSFs are in each case generated by guiding the light through optical pinholes. The optical pathway of a confocal microscope is illustrated in Fig.2.3. For an improved axial resolution, the detection pathway is particularly important: The confocal pinhole in front of the detector discriminates the out-of focus light and thus introduces optical sectioning.

The resolution of such a microscope is limited by the focal spot size of both the excitation and detection, which can both be determined by Abbe's law. Expressed in mathematical terms, the PSF of a confocal microscope is a combination of the excitation PSF $h_{\text {exc }}(\vec{r})$ with the detection PSF $h_{\text {det }}(\vec{r})$. As the detection pinhole in a real setup can not be assumed to be point-like but has a finite diameter, the detection PSF must be properly convolved with a pinhole function $o(\vec{r})$ generated by the back projection of 


\section{Theoretical Background}

the detection pinhole onto the sample. Accordingly, the confocal PSF is given by

$$
h_{\text {confocal }}(\vec{r})=h_{\text {exc }}(\vec{r}) \cdot\left[h_{\text {det }}(\vec{r}) \otimes o(\vec{r})\right] \simeq h_{\text {exc }}^{2}(\vec{r}) .
$$

Pursuant with the Rayleigh criterion, the resolution in z-direction of a confocal microscope is improved by a quadratic axial decrease of the fluorescence light not arising in the focal plane. The FWHM of the confocal PSF in the axial direction can be approximated by

$$
\triangle z=\frac{\lambda}{2 n \cdot \sin ^{2}(a / 2)} .
$$

The most frequently used setup nowadays is the confocal laser scanning microscope (CLSM), where the excitation and detection foci are shifted through the sample [184]. Thus, every single pixel can be addressed and questioned separately, if desired in all three spacial directions.

However, it has to be remarked that due to scattering and aberration effects in inhomogeneous samples causing a deformed and enlarged focus, the penetration depth of a confocal microscope is limited to the range of micrometers [183].

\subsection{High resolution fluorescence microscopy}

\subsubsection{The lateral direction}

A substantial progress in terms of the resolution of optical far-field microscopes took place in the beginning of the 1990s when Hell and Wichmann proposed the STED concept. The idea was to confine the fluorescence of the sample itself instead of the excitation light [69]. The first experimental realizations of targeted switching of the molecules from a fluorescent into a non-fluorescent state and subsequent readout were STED- and ground-state depletion (GSD) microscopy [104, 73, 67, 18] which in general refer to the RESOLFT concept [72]. Additionally, shortly there after several stochastic methods of switching came up independently, among them photo-activated localization microscopy (PALM) and stochastic optical reconstruction microscopy (STORM). Both approaches have proven to yield the long time desired nanoscopic resolution especially for biological samples with optical far-field setups and will be addressed in detail in the following sections.

\subsubsection{STED nanoscopy- an application of the RESOLFT concept}

The concept of Reversible Saturable Optical Fluorescence Transition (RESOLFT) relies on the probability to switch a fluorescent molecule (or more properly any system possessing an optically saturable transition, but this extravagates the limits of this thesis) optically controlled, saturable and reversible between a bright on-state A and a dark off-state B. The idea is to reduce the volume containing the markers in the bright state A below the diffraction limit, so that the effective excitation PSF is minimized. This can be done by a spatial intensity modulation of the switching laser beam with at least one 


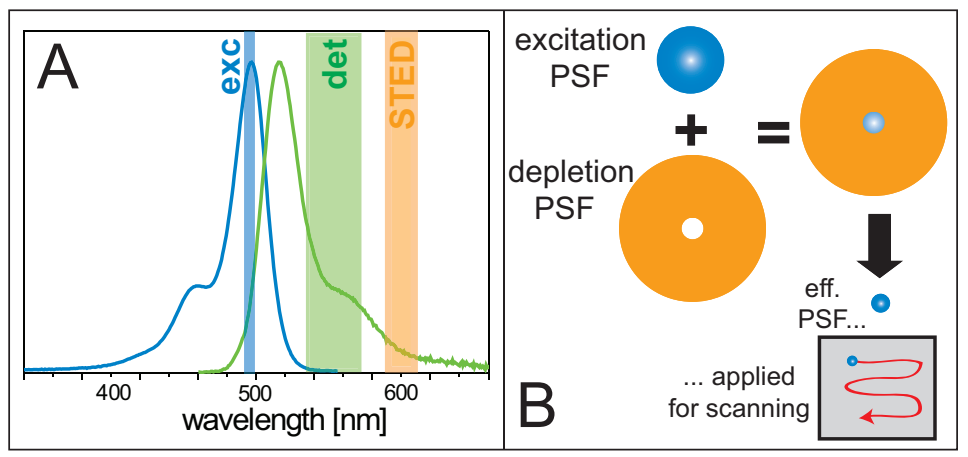

Figure 2.4: The principle of STED. A: Typical absorption (blue) and emission (green) spectrum of a visible fluorophore. The wavelengths applied for excitation and depletion are also indicated, as well as the detection region (green). B: The excitation PSF is superposed with the donut-shaped STED PSF, resulting in a laterally reduced effective excitation PSF, which is shifted over the sample.

intensity minimum.

In $S T$ imulated Emission Depletion (STED) microscopy fluorescent targets are used. The two level system is represented by the electronical ground state $S_{0}$ with its vibrational levels, equivalent to the dark off-state $B$ and the first excited state $S_{1}$, which represents the bright on-state $A$. The fluorophore is excited from $S_{0}$ to a vibronical excited state $S_{1}^{\text {vib }}$. Afterwards a relaxation to the vibronical ground-state of $S_{1}$ occurs within picoseconds. Without any modification the fluorophore would return spontaneously into $S_{0}^{\text {vib }}$ under emission of a red-shifted fluorescence photon and afterwards relax into $S_{0}$. This is the case for the zero-intensity region of the switching beam, and these photons are ideally fully detected. The wavelength of the depleting switching beam $\lambda_{\mathrm{STED}}$ is positioned at the long-wavelength end of the emission profile of the fluorophore, which is correlated with a transition into the topmost vibrational levels of $S_{0}$. To clarify this, a typical absorption and emission spectrum of a visible fluorophore is shown in Fig. 2.4. A. The according excitation and STED wavelengths, as well as the detection region are also indicated. By applying a high enough intensity, stimulated deexcitation is induced at the intensity-maxima of the switching STED-beam which leads to the simultaneous emission of two photons of the same wavelength $\lambda_{\mathrm{STED}}$. By placing sharp fluorescence filters into the detection pathway, those photons from the stimulated deexcitation at the red end of the emission spectrum are rejected.

In the experiment one has to bear in mind that the photophysical processes on the molecular level are much more complex than they are in a simple two-state system. Not only that the switching process is generally not describable as simply exponential. Moreover, especially under high beam intensities, additional processes of higher order or destructive photo-bleaching are getting more likely.

The best performance of such a minimized excitation spot in the experiment was treated theoretically by Keller et al [100]. A ring- or donut-shaped focus of the switching 


\section{Theoretical Background}

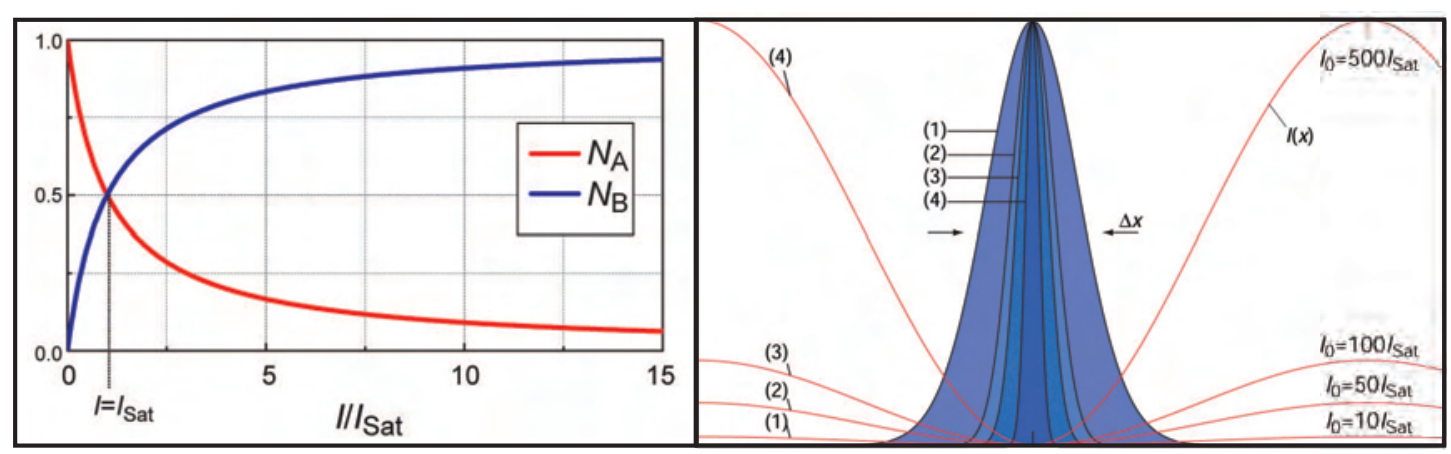

Figure 2.5: Left: Occupation probability of the two states A (red) and B (blue) in dependence on the applied intensities for stimulated transition form $\mathrm{A} \rightarrow \mathrm{B}$. At zero illumination intensity all molecules are in the bright state $\mathrm{A}$, with increasing intensity state B is populated and state A is depopulated. The intensity at which both states are equally populated is defined as the saturation intensity $I_{0}$. Right: Effect of the intensity of structured depletion light (red) with a zero in the center on the emitted fluorescence. The detected fluorescence spot (blue area) gets subsequently smaller.

beam overlapping the roundish excitation beam is the most efficient approach. This is illustrated in Fig. 2.4. B. Molecules or tiny objects at distances $<\lambda /(2 n \sin \alpha)$ can be resolved by scanning the combination of the excitation beam and the intensity zeros -and therefore the small effective excitation PSF- across them.

The successful resolution enhancement by RESOLFT is subject to several conditions. First of all, it is necessary that the switching process is optically saturable, which means that by applying a sufficient high laser intensity (almost) all molecules are switched. In contrast to what is observed at low switching intensities where the behavior is linear, the relative number of switched molecules in dependence on the applied intensity of the switching beam has to vary. This non-linear behavior, which is compulsory to saturate the transition between the two states, is illustrated in Fig.2.5.

The saturation is mathematically indicated by the depletion factor $\eta\left(I_{\mathrm{STED}}\right)$. It is the ratio of the number of molecules in the on-state A after employing a depletion beam with the power $I_{\mathrm{STED}} N_{\mathrm{A}}\left(I_{\mathrm{STED}}\right)$ and the number of molecules in A without a depletion beam $N_{\mathrm{A}}(0)$ :

$$
\eta\left(I_{\mathrm{STED}}\right)=N_{\mathrm{A}}\left(I_{\mathrm{STED}}\right) / N_{\mathrm{A}}(0) .
$$

Hence the initial excited population is reduced by the factor of $\eta$. In some context the depletion probability or saturation function is used, which is given by $(1-\eta)$. The intensity, at which $50 \%$ of the molecules are switched and an equal number of molecules initially excited is in states A and B respectively, is called saturation intensity $I_{\text {sat }}$ with $\eta\left(I_{\text {sat }}\right)=0.5$.

Assuming a simplified exponential relation between the switching intensity $I_{\text {switch }}$ and the number of molecules in the on state $N_{A}$, the achievable resolution by RESOLFT is 
described by eqn. 2.9 .

$$
\Delta x, \Delta y \approx 0.61 \frac{\lambda}{2 n \cdot \sin \alpha \cdot \sqrt{1+a I_{\text {switch }} / I_{\text {sat }}}} .
$$

The variable $a$ denotes the geometry of the intensity distribution around its central minimum of the switching beam [58, 68]. It turns out that for an arbitrary high switching intensity $I_{\text {switch }}$, an infinitesimal resolution is achievable, as long as the switched marker is small enough and photobleaching is not an issue.

Though the excitation PSF is not diffraction limited anymore, the detection still is. To enable a feasible SNR, every marker molecule is questioned several times. Thus, the switching process has to be reversible. Anyway, in contrast to the $\mathrm{A} \rightarrow \mathrm{B}$ transition, the reverse process does not need to be optically induceable, but can be spontaneous, thermally or chemically driven.

Further, it is important to account the processes of stimulated and spontaneous emission as competing during the STED-process. This implies that primarily a sufficient long lifetime of $S_{1}$ (typically $\tau_{\mathrm{f}}=1-5 \mathrm{~ns}$ ) is required. For a resolution enhancement, the offswitching has to be performed before the spontaneous emission process sets in. In other words, the rate constant $k_{\mathrm{STED}}$ for stimulated emission, defined as $k_{\mathrm{STED}}=\sigma_{\mathrm{STED}} \cdot I_{\mathrm{STED}}$, where $\sigma_{\text {STED }}$ is the interaction crossection and $I_{\text {STED }}$ the intensity of the STED beam, has to be significantly higher than the rate constant $k_{f}$ for spontaneous emission.

Second a fast degradation of $S_{0}^{\text {vib }}$ within picoseconds is mandatory. Would the offswitched molecules stay on a vibronical excited level $S_{0}^{\text {vib }}$, a reexcitation by the intense STED-beam would prevent the effective depopulation of the $S_{1}$ state and therefore the off-switching. Besides, if the intensity of the excitation beam is not sufficiently high to depopulate the ground-state effectively, reexcitation still becomes a problem. The amount of molecules populating the higher vibronic levels of the ground-state due to the Boltzmann distribution is negligible at room temperature $(\sim 298 \mathrm{~K})$.

Third the intensity of the STED light has to be high enough to perform an effective fluorescence inhibition. For $\lambda_{\mathrm{STED}} \simeq 600 \mathrm{~nm}$, the latter has to adapt values $\gg 33 \mathrm{MW} / \mathrm{cm}^{2}$, typically in the range of $\mathrm{GW} / \mathrm{cm}^{2}$.

A lot of impressive progress in the lateral resolution enhancement has been made. This includes the use of continuous wave (CW) lasers in contrast to pulsed ones, which are available for many more colors [182. Additionally, STED was shown to be compatible with multi-color- [26] and life-cell- [64] schemes.

But in general, any two-state system can used for resolution enhancement via RESOLFT as long as the switching process is optically saturable and reversible and as long as the on-state is detectable. Apart from STED, it has successfully been applied in GSD microscopy, where the off-sate is represented by the triplet state of a molecule, and with photoswitchable dyes and proteins [83, 18, 151, 163, 3]. In case of the latter, isomerisation reactions of the chromophores of photoswitchable FPs or dyes are drawn.

Due to the comparably long lifetime of either the triplet or the isomerisation state, essentially less laser power needs to be employed in comparison to STED microscopy. Owing to the low switching intensities required, the method were implemented in widefield 


\section{Theoretical Background}

setups for fast image acquisition. Problematic are on the other hand the low fluorescent quantum yield of FPs and their sensitivity to destructive photoreactions, even at low laser intensities.

Some related methods to the RESOLFT concept are saturated structured illumination microscopy (SSIM) and saturated pattern excitation microscopy (SPEM), although they do not employ the characteristic donut shaped switching pattern described above 65, 55]. Both techniques use a wide-field scanning setup that is highly parallelized. By creating a standing wave interference pattern of the excitation light, intensity maxima and minima are induced. Shifting these line-shaped zeros through the sample stepwise and sequentially reading out the fluorescence with a camera after every single step produces a one-directional high resolution image. Tilting the sample covers all directions in the focal plane, but subsequent mathematical analysis is strongly required. However, the combination of spectral precision distance microscopy/spectral position determination microscopy (SPDM) and spatially modulated illumination (SMI) was reported to give a single molecular resolution as well [111].

\subsubsection{Fluorescence nanoscopy by stochastic methods}

Theoretically, for sparsely enough labeled structures (with an intermolecular distance $<200 \mathrm{~nm}$ ), a molecular localization on the nanoscale can be achieved by rather simple optical methods, assuming that the single fluorescent label emits enough photons. In fact, most interesting structures if efficiently labeled are more dense. In stochastic high resolution methods, a fine object is obtained by stochastic on-switching of only a small fraction of the labels originally residing in the off-state. The on-state of the labels can either be induced by absorption of a photon (photoactivation in PALM [75], STORM [144], dSTORM [63], FPALM [95], PALMIRA [31, 14]) or by spontaneous relaxation from an optically created non-fluorescent off-state like the triplet state (GSDIM [38, 162]). As long as a certain amount of $m$ detectable photons is emitted through repeated excitation from a single label before it returns to an off-state and as long as the molecules in the onstate are at least $>\lambda /(2 n \sin \alpha)$ apart from each other, many individual molecules can be localized simultaneously as single diffraction spots on a camera. Centroid calculations on the frames of several consecutive switching cycles renders a considerable number of localized molecules, where center positions finally form the image. As the precision varies statistically with $m$ in terms of $\lambda /\left[(2 n \sin \alpha) m^{1 / 2}\right]$, a brightness threshold $M \leq m$ is defined to reduce the background artifacts and at the same time enhance the average resolution. In the case of no background, $M=m$ can be assumed, which gives the highest possible resolution. In fact, with sophisticated setups a resolution of up to $20 \mathrm{~nm}$ in all three dimensions and with up to three different colors has been shown so far [154]. Stochastic methods are in general working with low switching intensities, going along with a linear excitation behavior, and without the need of many switching cycles per emitter.

Still, and against various claims, the emission of $m$ photons cannot be expected to be linear, and thus stochastic methods are as well working with non-linear effects.

Apart from ultra fast cameras ( $500 \mathrm{~Hz}$ frame rate) and fluorophores that ensure large 
values of $m$ per switching cycle, for two dimensional imaging no sophisticated setup is required. In special cases the system is even reduced to a single needed laser and an independently running camera (GSDIM, PALMIRA). Therefore, although mathematical data analysis is mandatory and the selection of the switching intensity needs careful consideration -high intensities produce too many molecules in the on-state, while low intensities slow down the acquisition time- stochastic methods seem to be the technically simplest far-field nanoscopic methods.

However, STORM imaging works with organic fluorophores, which are not membranepermeable, and is hence limited to few applications, especially in terms of life cell imaging.

For PALM, which in contrast to STORM was shown to work with fluorescent proteins, the low illumination intensities enable imaging within a living cell. However, high resolution of dim structures comes with a long acquisition times and thus fast processes in living specimen are not accessible.

As a conclusive remark, it is worth to mention that a more effective spatial resolution calls for a coincidental more effective labeling, which is particularly relevant in biological applications. This has been formulated by Shroff and coworkers in analogy to the Nyquist criterion [35. The distance between two labeled molecules must be smaller than half of the desired spatial resolution to obtain an integrated image. If the resolution achieves a value of $5 \mathrm{~nm}$ for instance, the two labels should be as close as $2 \mathrm{~nm}$. This demand is not combinable with the bulkiness and the weak affinity of most common antibodies and therefore poses new challenges for biologists and chemists in terms of labeling strategies.

\subsubsection{The axial direction}

\subsubsection{Optical sectioning methods}

Achieving an improvement in the axial resolution of optical far-field microscopes has for a long time been a challenge. According to Abbe's prediction, it has always been the weaker point with respect to the investigation of three dimensional samples. Thus, several optical concepts aiming at a refined axial resolution, have been established: confocal microscopy, multiphoton microscopy, TIRF microscopy, $4 \mathrm{Pi}$-microscopy and $\mathrm{I}^{5}$-microscopy. They are commonly referred to as optical sectioning methods and outline in the following Subsections. Still, achieving an axial resolution below $100 \mathrm{~nm}$ was for a long time hardly possible.

Multiphoton microscopy The most common mode of multiphoton-microscopy is twophoton microscopy. It was predicted in 1931, but not successfully applied before 1962 and uses the non-linear effect of simultaneous absorption of two photons 23. The concept is based on the idea that two combined photons, each of them having half the energy required for excitation, can excite a fluorophore, resulting in the emission of a fluorescence photon (see term scheme in Fig. 2.6, A). Therefore, the probability of effective absorption depends on the square of the intensity of the incoming light and as a consequence a confined excitation PSF is created. The used infrared light is much less affected by scattering and enables a penetration depth of up to $100 \mu \mathrm{m}$ into cellular tissue which is 


\section{Theoretical Background}

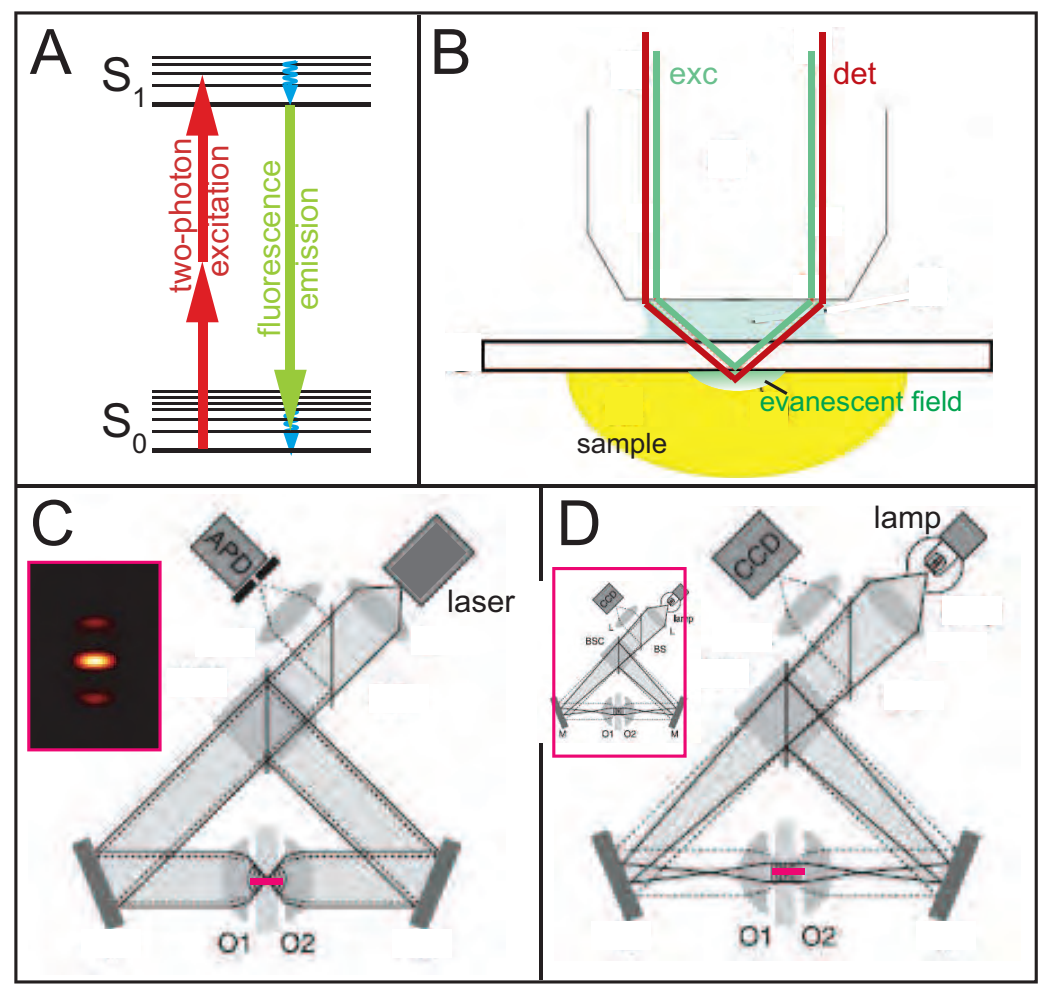

Figure 2.6: Optical sectioning approaches. A: Scheme of two-photon-excitation. The excitation light has about twice the wavelength of the fluorescence light. B: TIRF-microscopy. Focusing the excitation light at a high angle onto the sample surface yields an evanescent field (green). Fluorescence excitation is limited to this volume. C: Setup of a confocal type C 4Pi microscope with two opposing lenses. The effective imaging PSF is also shown. D: Setup of a widefield $\mathrm{I}^{5}$ microscope with two opposing lenses; the effective imaging PSF is shown. D and $\mathrm{C}$ are adapted from [13].

beneficial in many biological applications. However, the size of the excitation spot is not decreased to the longer wavelength of the light.

Thus, the major weight is not on a resolution refinement with regard to the focal volume, but on the induction of focal planes at large distances from the sample surface. In addition, while little photobleaching is observed in the out-of focus region, the required light intensities in the focal spot are even more destructive.

However, since two-photon microscopy is nicely compatible with life-cell imaging, it has become quite popular.

TIRF microscopy Total internal reflection (fluorescence) microscopy (TIRFM) is, in contrast to two-photon-microscopy, only suited for studies on the direct periphery of the coverslip. Moving the investigated layer along the optical axis through the sample is not 
possible. The working principle of a TIRF microscope is sketched in Fig. 2.6, B. The sample interface is illuminated at a high incident angle such that total reflection occurs. This establishes an evanescent light field at the sample's surface. The penetration depth of the evanescent field is restricted to about $100 \mathrm{~nm}$, and thus only molecules located in a very thin layer close to the coverslip are illuminated and detected. At a larger distance (more than $100 \mathrm{~nm}$ ) the intensity of the excitation light is negligible which excludes distracting fluorescence signal from deeper layers of the sample [4].

Clearly, the restricted imaging depth is the severest disadvantage of the technique. However, in biology, it is often used to study processes related to the plasma membrane of fixed and living cells.

4Pi microscopy In 4Pi microscopy the axial resolution of a scanning microscope can be improved by a factor of $3-7$ with two opposing lenses of high numerical aperture. A typical 4Pi-setup is illustrated in Fig. 2.6. C. At the common focal point of the lenses the two counterpropagating spherical wavefronts of the excitation light are coherently summed. The same applies to the wavefronts of the emitted fluorescence light on the detector. Consequently, the observation angle of previously $\sim 73^{\circ}$ is enhanced. In other words an essential expansion of the aperture solid angle is created. Additional mathematical deconvolution of the data might be either optional or mandatory [66, 12].

However, as the sample is placed between the two opposing lenses which both have a certain working distance, $4 P i$ microscopy is restricted to samples which are less than $200 \mu \mathrm{m}$ thick.

A combination of $4 P i$ microscopy with two-photon excitation was also reported [47].

$\mathbf{I}^{5}$-microscopy Incoherent illumination imaging microscopy $\left(\mathrm{I}^{5} \mathrm{M}\right)$ uses as well as in 4Pi-microscopy two opposing objectives, but is a widefield technique. An I ${ }^{5} \mathrm{M}$ setup is sketched in Fig. 2.6. D. A simple fluorescence lamp is employed for coherent Köhler illumination through both lenses, yielding a standing wave. By separated interference effect of the excitation and the detection light, the signal from an object is enhanced, while the one from its periphery is extinguished. A seven-fold resolution enhancement was reported this way. However, in $\mathrm{I}^{5}$ microscopy, mathematical deconvolution can not be circumvented [56].

A closely related technique is $\mathrm{I}^{5} \mathrm{~S}$ microscopy, where the $\mathrm{I}^{5}$ microscopy is combined with structured illumination microscopy to enhance the lateral resolution as well. The achieved resolution was reported to be $100 \mathrm{~nm}$ in all three dimensions [152].

With the further refinement of the lateral resolution in optical microscopes, also the efforts on the axial resolution experienced further stimuli. A combination of these new methods with the reported optical sectioning concepts is more than evident. In consequence, several more or less sophisticated approaches have recently been reported, partly reliant on subsequent mathematical reconstruction algorithms. In addition, some new concepts came up which basically achieve a resolution enhancement by further refinement of either the excitation or, more common, the detection light. The complexity of 


\section{Theoretical Background}

these methods increases with the achieved resolution. A brief overview is given in the following.

\subsubsection{Optical sectioning and high resolution microscopy}

Axial STED microscopy The concept of RESOLFT has been shown to work in the axial direction as well To this end, instead of a lateral donut shaped STED beam, an axial depletion pattern is generated. This way the fluorescence light from the upper and lower parts of the confocal excitation focus is depleted. The axially reduced excitation PSF can be scanned three dimensionally through the sample, resulting in an axial resolution of $80 \mathrm{~nm}$. Meanwhile, the detection in the lateral direction is still mostly diffraction limited. Subsequent acquisition of an additional scan of the sample with a laterally reduced focus would in the end enable a three dimensional high resolution image, but was so far hindered by photobleaching. [59, 64].

4Pi-STED microscopy 4Pi-STED microscopy combines the concepts of 4Pi-microscopy and STED microscopy. A 4Pi- setup with two opposing objectives is to this end used. While the excitation and detection is performed through one lens only, the depletion pattern is generated by counterpropagating, aberrated wavefronts focused through both lenses. Scanning is performed by moving the sample through the refined excitation spot. This ended up in an axial resolution of about $33 \mathrm{~nm}$ and was in this respect path breaking [28, 29.

iso-STED microscopy A further advancement of 4Pi-STED microscopy is the concept of isoSTED [147, 170]. Again, two opposing objectives are used in the setup. In contrast to $4 \mathrm{Pi}$-STED microscopy, not only the STED pattern, but also the excitation and the detection are performed through both lenses. This results in an effective spherical nanosized excitation spot with a diameter of $45 \mathrm{~nm}$. Thus, a resolution enhancement down to the nanoscale is demonstrated simultaneously for all three spatial directions. Since it is a noninvasive approach, it is potentially combinable with life-cell-imaging. However, the method requires high laser intensities which are applied to the sample. Therefore, up to now photobleaching in the peripheral layers of the focus severely limits the entire investigation of a structure.

2-Photon-STED microscopy Recently a combination of STED microscopy with twophoton excitation was reported [129]. The achieved lateral resolution is about $30 \%$ poorer than for other STED methods. This is because the long wavelength of the excitation light cannot fully be compensated by the reduced focal spot size of a two-photon setup. Thus for fluorescent beads the declared resolution was about $50 \mathrm{~nm}$ laterally.

As typical for multiphoton approaches, the axial resolution is mainly refined in terms of the penetration depth into moderately scattering tissue than by a refinement of the focal spot. This enables the addressing of deeper layers of a three dimensional sample as it would be possible for single-photon excitation concepts. 
PALM and STORM Since the PALM and STORM concepts use widefield setups, the axial resolution is intrinsically expected to be quite poor. This is the reason why the first reported setup in either technique was a TIRF setup.

For the concept of STORM fluorescently labeled DNA fragments on a coverslip were used to first demonstrate a resolution in the range of $40 \mathrm{~nm}$ laterally [144]. In contrast, PALM imaging was performed either in the very periphery of a whole cell or on thin cryosections, resulting in a lateral resolution of about $25 \mathrm{~nm}$ [11]. For the latter fluorescent proteins were used as markers, while STORM utilized organic switchable fluorophores. In both cases the axial resolution was limited by the evanescent field of the excitation light.

Still, the omnipresent requirement of a refined axial resolution stimulated the invention of several more advanced concepts. Most of them rely on sophisticated refinements of the detection pathway, but sometimes also the excitation pattern is specifically engineered. However, none of them performs without subsequent computational image formation.

2-Photon PALM A combination of two-photon-excitation with PALM enabled to image fluorescent molecules up to $100 \mu \mathrm{m}$ deep in $400 \mu \mathrm{m}$ thick tissue slices. Simultaneously, in the lateral direction, a resolution of $50 \mathrm{~nm}$ was achieved by the PALM mode [173.

A similar approach using continuous wave (CW) lasers in contrast to the normally applied high pulse rate lasers and photoswitchable rhodamines reported a lateral resolution of $15 \mathrm{~nm}$ in the focal plane, which was generated by two photon excitation [37.

Biplane FPALM In biplane (BP) FPALM, a normal PALM setup with a modified detection path, which allows for simultaneous detection from two axially separated object planes, is applied. The signal from the two recorded regions of interest can be combined into a 3D raw data stack consisting of two planes. This is sufficient to localize single particle which have a sparse distribution and are closely located to one of the two planes. The observed resolution was $20 \mathrm{~nm}$ in the lateral and $70 \mathrm{~nm}$ in the axial direction respectively [95].

3D-STORM With 3D-STORM an enhanced axial resolution down to $20 \mathrm{~nm}$ is generated by using astigmatism [86. A cylindrical lens is introduced into the detection path to create a different focus in the $\mathrm{x}$ - and $\mathrm{y}$-direction respectively, such that the image of a molecule appears elliptical. This ellipticity varies with the $\mathrm{z}$ position of the molecule. By subsequently applying a mathematical image reconstruction, this variation can be used to derive the value of $\mathrm{z}$ with high precision. Imaging is performed by shifting the focal plane along the optical axis. The lateral resolution was as well reported to be $20 \mathrm{~nm}$.

3D-PALM This approach generates a three-dimensional resolution by sophisticated shaping of the excitation pattern. Using a double-helical PSF for example resulted in a resolution below $20 \mathrm{~nm}$. The PSF has two dominant lobes in the image plane whose angular orientation rotates with the axial $(\mathrm{z})$ position of the emitter. Finding the center 


\section{Theoretical Background}

of these lobes enabled the precise localization of the emitter in a sample up to $2 \mu \mathrm{m}$ thick [134].

iPALM In interferometry (iPALM) a complex multiphase interferometric setup is applied to perform PALM imaging. The reported resolution on thick samples was declared to be below $20 \mathrm{~nm}$ in all three dimensions. Similar as in $4 \mathrm{Pi}$ and $\mathrm{I}^{5}$ microscopy, two opposing lenses are used for the detection of fluorescence events. Thus, an emitted photon can simultaneously travel along two distinct optical paths, which are subsequently recombined, so that the photon interferes with itself. This is used to recalculate the original localization of the photo-event [154].

\subsubsection{Mechanical sectioning}

Although an optically performed axial resolution of up to $20 \mathrm{~nm}$ is not utopistic anymore, all optical concepts suffer from a major drawback. Due to scattering and aberrational effects they are severely restricted by the penetration depth of visible and infrared light, in particular when thick, inhomogeneous samples like tissue blocks are to be observed. This calls for another kind of resolution refinement which is not directly related to the focal spot size.

To a certain extent, semi-thick mechanical sectioning to a thickness of several micrometers addresses this problem. It is quite convenient because it works without embedding the sample into a plastic block or freezing it and was also shown to be combinable with life cell imaging.

In contrast, mechanical ultrathin sectioning of specimen to a thickness of several tens of nanometers was by default only used for transmission electron microscopy. It is mandatory there because the penetration depth of an electron beam is even more restricted than that of a light beam. For optical microscopy this has been only rarely applied [123, 117].

However, mechanical ultrathin sectioning generates a resolution refinement on two counts: It reduces the fluorescent volume within the optical focus, which can be interpreted as an effectively reduced axial focus. In addition it enables the wholistic access to an arbitrary thick sample with high resolution. In this regard, at the current point of research optical sectioning methods can not compete with the mechanical ultrathin sectioning. 


\subsection{The mechanical sectioning approach}

\subsubsection{Ultramicrotomy}

In the early 19th century studies of natural tissue were facilitated and improved due to the advances in the construction of light microscopes. Still, the constitution of a three dimensional object was not truly accessible without looking at its interior. therefore, anatomists and microscopists started to cut their samples into thin sections using razor blades. Unfortunately this was not always sufficient to get a good transmission of light under the microscope.

One initial idea to overcome this problem was to squeeze the thinness of the sections further which was of course not very conducive for structural investigation [139. A fundamental improvement of mechanical sectioning was done by Wilhelm His in 1865 [80, who refined the microtome, a precise cutting equipment first described in 1770 by Hill [77. In fact the true father of the cutting tool is hardly under discussion, since the name "microtome" was not created before 1839 and several other names like "cutting engine" were used in various contexts as well [87].

Particularly due to the enormous contemporaneous progress in the field of optical microscopy in central Europe, a lot of improvements of the device followed, including variations concerning the knife and its angle and the operating mode as well as improvements in the sample preparation itself. Already at the end of the 19th century, this interplay lead to a resolution which was about one magnitude higher [120].

During the next century, light microscopists searched mainly for optical approaches to overcome the axial resolution problem. An overview has been given in the previous Chapter. Further approaches based on mechanical sectioning were quite sporadic [123, 125.

Else it was in electron microscopy and particularly in transmission electron microscopy (TEM) which was developed in the early 1930s [105]. To obtain a resolution on the molecular scale (or even up to $0.1 \mathrm{~nm}$ ), an electron beam is focused onto the sample instead of light. Like all matter, electrons have both wave and particle properties (as theorized by de Broglie [19]). Their wave-like properties mean that a beam of electrons can be made to behave like a beam of electromagnetic radiation. The electrons are very sensitively scattered by any atom, whereupon proton-dense nuclei with a high atomic number scatter the electrons most. This is also why working under vacuum conditions is essential. The direct observation of the refocused and magnified electron beam on an imaging device, such as a fluorescent screen, a layer of photographic film, or a sensor (e.g. a CCD camera) yields a TEM image where proton-dense structures become visible. To get a good imaging contrast, appropriate samples have a thickness that is comparable to the mean free path of the electrons traveling through the sample, virtually a few tens of nanometers in case of high quality samples.

Several advanced methods exalt the image quality in terms of contrast for certain applications, like diffraction, electron energy loss (EELS) [78] or phase contrast imaging. Still, controlling an electron beam in a microscope is more demanding than it is for a beam of light. therefore,, technological changes on the setup aiming for an improved 


\section{Theoretical Background}

(axial) resolution are less accessible and thus not promising. Hence, for biological samples which have a quite uniform and dense distribution of atoms with similar atomic numbers, it is particularly mandatory to have a certain thinness. This is still despite the fact that membrane staining with heavy metals, e.g. lead acetate or uranyl acetate, might visualize a variety of cellular components and enhance the imaging contrast [175, 157, 108].

All the more, cutting material into precise ultrathin sections posed and still poses a challenge, especially for soft biological material. The earlier microtomes were improved upon further and the first so called ultramicrotome was presented in 1951 by Porter and Blum[106.

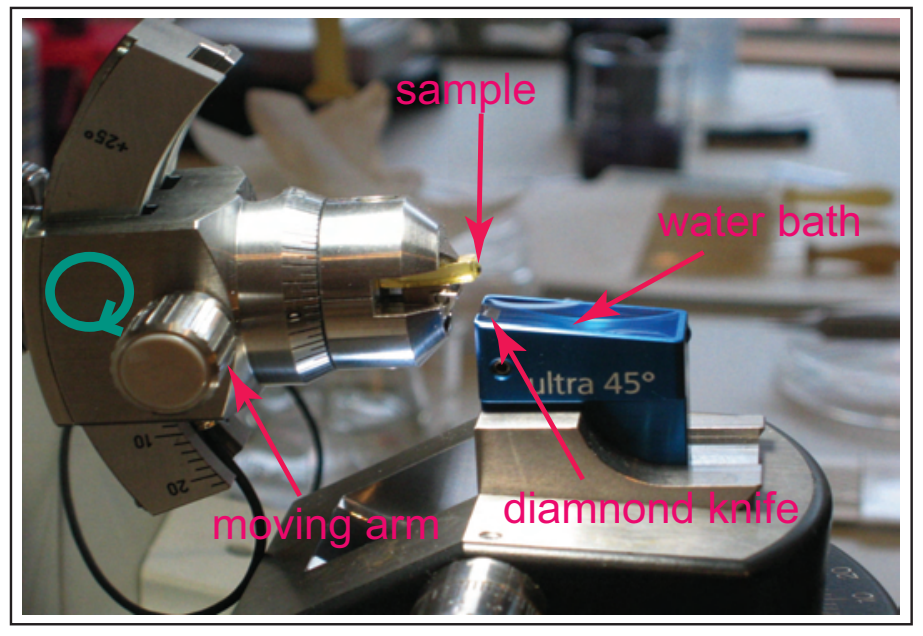

Figure 2.7: Picture of a microtome. The trimmed samples are placed onto a moving arm, that performs a circular movement, passing the diamond knife once during each cycle. The diamond knife is equipped with a waterbath to collect the slices.

Ultramicrotomes are in general based on a so called rotational working mode, where a diamond knife is stepwise moved towards a rotating arm by a (thermally) precisely controlled header. The previously trimmed sample with a front-area of about $1-4 \mathrm{~mm}^{2}$ is fixed on this arm, passing the knife after each rotation. A picture of a microtome unit is shown in Fig. 2.7. Nowadays uniform ultrathin sections of $10 \mathrm{~nm}$ to $500 \mathrm{~nm}$ thickness are reliably obtained. However, the most accurate sectioning can be achieved with the recently introduced ultrasonic diamond knives. These knives perform a lateral movement during the sectioning of a sample, similar to cutting bread. The moving speed can be adapted to the sample. Thus, even softer polymer blocks can be easily sectioned without squeezing the sample and deforming the section. However, ultrathin sectioning with least possible structural disruption unambiguously requires hard samples.

For performing the cutting process at room temperature, embedding the sample into a plastic block is the preferred method. Nowadays this approach is complemented by cryomicrotomy at low temperatures, where the demanded hardness is achieved by freezing the sample. 
Despite the fact that one or the other sectioning method has to be balanced and also, that one might still come across analogical difficulties and drawbacks, investigating ultrathin sections with new high resolution light microscopes is rather obvious. This is for two reasons: First, new limitation were revealed for the imaging of dense structures, which had at a poorer resolution so far been dwarfed by a blurred spot. Second, offering a nanoscopic resolution by light microscopy paves the way for numerous studies which were so far dependent on electron-microscopic investigation. Additionally, the labeling advantages in light microscopy even broaden the spectrum of possibilities in research.

\subsubsection{Ultramicrotomy at room temperature}

Cutting ultrathin sections at room temperature is the older and less sophisticated approach. It strictly presupposes the embedding of the sample into a plastic block, which is hard enough for ultrathin sectioning. The workflow for the preparation of a resin embedded samples is illustrated in Fig.2.8. The embedding process consists of the infiltration with the monomer solution and the hardening reaction itself. Depending on the polymer type, this is preceded by a stepwise replacement of the aqueous solution with an organic solvent. After the polymerization of the plastic in an adequate mold, the block is at the site of the sample trimmed to a small surface, normally a few square millimeters.

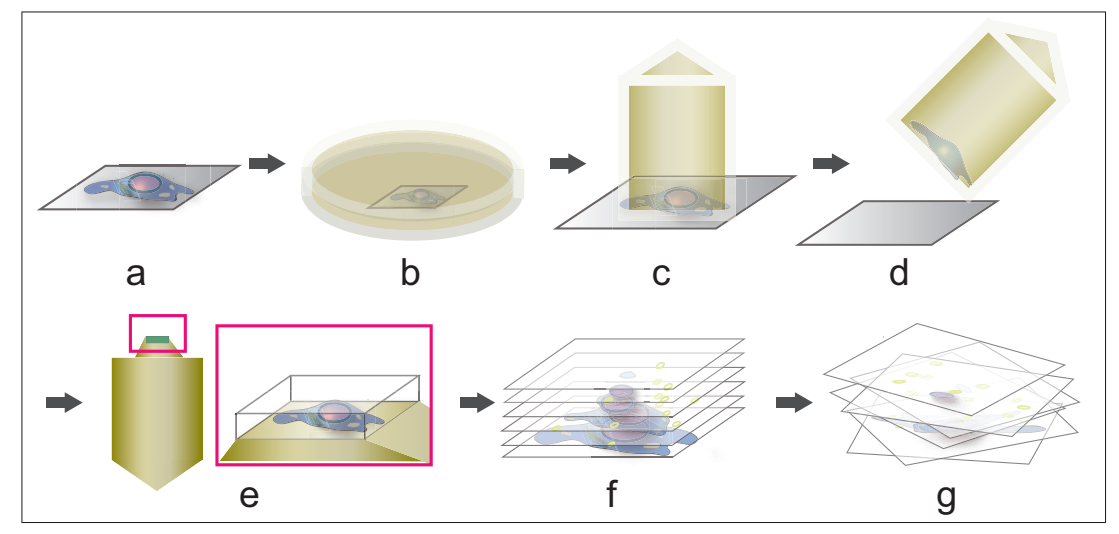

Figure 2.8: Workflow for utramicrotomy at room temperature: (a) cultured, fixed samples are infiltrated with the monomer solution (b); (c) polymerization of the resin block in a suitable mold; (d) detaching of the polymer block with the embedded sample from the coverslip and mold (by either liquid nitrogen or hydrofluoric acid); (e) trimming of the block surface; (f) ultrathin sectioning with an ultramicrotome; (g) investigation of the individual (randomly orientated) sections.

At the ultramicrotome, the diamond-knife used to cut the sample is equipped with a water bath. The water is directly attached to the knife-edge. This forces the cut sections to slide directly onto the water surface. From here they can be fished out afterwards with a wire-loop. This procedure has the advantage that the surface tension of the water 


\section{Theoretical Background}

stretches the sections and keeps them in good shape. Moreover, the sections stay together at their edges, forming a band, which allows one to pick a small series of sections at once.

Resin embedding and cutting at room temperature has some advantages over cryomethods. At the site of the sample it is not limited to a certain size, as long as a thorough monomer-infiltration and complete polymerization is ensured. This makes any part of an animal or plant accessible for high-resolution investigation by either optical or electron-microscopic approaches.

In addition, it is particularly interesting for optical methods, since the labeling with fluorescent dyes can be performed before the embedding. This holds for genetic encoding methods with FP, as well as for immuno-staining of the fixed sample. If postembedding staining with metal particles is necessary, the preservation of antigen sites gains importance. This competes with the chemical polymerization reaction, which might affect them to a certain extent. Moreover in post-embedding methods, only the very outer of the section is accessible for the staining target.

However, if a fluorescent label is added to the sample before embedding, it has to be considered that the fluorophore itself is affected by the dehydration and polymerization procedure.

Finally, ultrathin sectioning of resin embedded samples was recently shown to be automatable by Lichtman and coworkers. A large stack of sections is collected on a plastic tape guided through the waterbath behind the knife instead of fishing them off the surface by hand [61. Room temperature cutting is less tricky than cryo-sectioning, but it is still laborious. therefore,, without automation, the thickness of a serial stack of sections which can be cut is severely limited. Automatization the cutting paves the way to larger tissue blocks or even whole organs, like e.g. brains, to be serially sectioned and afterwards reconstructed.

\subsubsection{Cryo-microtomy}

A newer and more sophisticated idea for ultrathin cutting is to harden the sample by freezing it. This implies that the cutting has to be performed at low temperature as well. therefore,, a cryo-chamber cooled with liquid nitrogen is employed. This approach is called cryo-microtomy [89].

Before freezing, the sample is infiltrated with an aqueous solution of sucrose. This effectively prevents the formation of ice crystals during freezing and, with it, a potential disruption of the sample [169]. As trimming the sample is not easily possible after freezing, the sample has to be shaped beforehand. To make it more handy, it is additionally sometimes embedded in gelatin or agarose.

As taking up the sections in a water-bath is not possible at low working temperatures, the cut sections hang in the air. Taking them from the knife-edge is performed by attaching a droplet of sucrose solution in a metal loop to them very face. This makes it much harder to get the sections off well-preserved.

An additional problem of cryo-sectioning is the electrostatic loading during the cutting process which makes the samples just fly off the knife from time to time. 
therefore, it is obvious that, in addition to the need of an advanced setup with a cryochamber, the sectioning process is much more laborious. Obtaining even a small series of sections of a sample needs a lot of training. In addition, the frozen sample as well as the cut sections are more sensitive than are plastic blocks, which makes them harder to store or handle.

However, the main advantage of cryo sectioning is that it works without an embedding process. It has been observed that during cryo-sectioning much more epitopes are preserved for post-embedding antibody-staining, which is crucial for specific protein labeling and imaging with TEM [115]. This is mainly because the amino-functions of the proteins are not affected by any fixation or polymerization process.

In combination with newly established fixation methods like rapid or high pressure freezing (HPF), which even works without chemical fixation with para-formaldehyde (PFA) or glutaraldehyde (GA) in advance, freezing is nowadays the gentlest and most structure-preservative way to get a sample ready for ultrathin-sectioning [27].

In any case, (shock-) freezing is limited to very thin (monolayer) samples, whereas high-pressure freezing requires the samples to be housed in a $1-2 \mathrm{~mm}$-sized holding chamber. Thus, larger volumes or even whole organs are not suited for freezing methods.

\subsubsection{Embedding polymers}

For the already discussed reasons, polymer embedding was chosen to enable the preparation of ultrathin sections for high-resolution light microscopy. The number of organic polymers available on the market is nearly endless. This leads to the question which polymers are suitable for ultrathin sectioning and at the same time for the embedding of cellular structures [1].

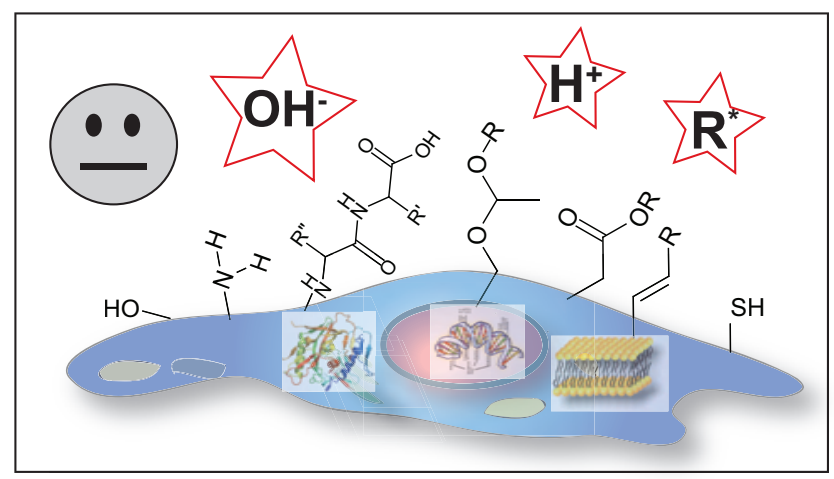

Figure 2.9: Functional groups in cellular tissue which can potentially react with the starter molecules commonly used in polymerization reactions.

On the side of ultramicrotomy, the requirements are pretty simple. First, the resin has to be hard enough to be sectioned ultrathin, but it must not be brittle. Soft plastics give way to the knife and are if at all only suited for sections with a thickness in the range of micrometers. 


\section{Theoretical Background}

Second, the monomers have to be small enough to penetrate the sample. If the structure is not thoroughly infiltrated, the polymerization will be incomplete which causes damages during the sectioning procedure.

Thirdly, the polymerization should proceed with low shrinkage, or otherwise also the structure to be observed will be squeezed.

The demands for cellular structures are a bit more strenuous. As the focus of investigation in high resolution methods is the ultrastructure of the sample, it ideally should not be affected at all. But cellular tissue contains a lot of sensitive functional groups, which are destroyed by the activated monomers or catalysts if the reaction conditions are too harsh. The main functional groups, which are in this regard important are depicted in Fig. 2.9. Since many of these side-groups have essential functions in the arrangement of cellular components, for example, the folding of proteins or the DNA, it is obvious that the reaction conditions should be as mild as possible. Ideally, the added monomers carry more reactive functional sites, which in consequence preferably react in the polymerization process.

Certainly, the involvement of the biological structure can not be completely avoided, especially, as a thorough through-out polymerization of the plastic is essential for ultrathin sectioning. To a certain extent, it can be protected by fixation with aldehydes which react with amino- and hydroxy-functions, or other agents like $\mathrm{OsO}_{4}$ which react with C$\mathrm{C}$-double bonds in the lipid membranes. The reaction of the fixation agents is much more predictable. They form an even network around the original structures instead of deforming them.

However, to a certain extent it is also useful if chemical bonds are formed between the polymer and the cellular structure or, even better, the through the fixation constituted network. This ensures that the cellular components are not washed out of a sections after ultrathin cutting.

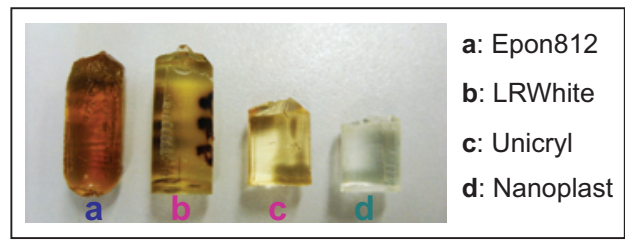

Figure 2.10: Photo of four different common plastics used for ample embedding and subsequent ultrathin sectioning. The color is indicating the different properties of different resin classes. (a) epoxide resin; (b),(c) acrylate resins; (d) melamine resin.

How much the cellular ultrastructure is finally manipulated by the polymerization reaction depends is not only subject to the added chemicals, but also to the further reaction conditions. Heating proteinogenic structures to temperature far higher than $60^{\circ} \mathrm{C}$ will certainly result in their degeneration. Thus this is to be avoided. The same holds for polymerization after UV light initialization, especially when the samples were 
pre-embedding stained. Intensive UV radiation often causes substantial photobleaching.

As cellular structures are normally in an aqueous environment, dehydration of the sample is unfavorable. This is foremost because hydrophobic cellular components, for instance lipid membranes, are washed out by organic solvents. In addition, FP lose their fluorescence capability when the crystal water in their periphery is extracted and therewith the hydrogen-bonding network is destroyed. Also the polymerization of many monomers is interrupted when water is present. Thus, in a distinct experiment, it has always to be considered to which extent the loss of water can be tolerated in favor of a certain polymer.

Polymer embedding of biological samples is crucially important for TEM and hence the development of suitable plastics is well-explored. Thus, a huge variety of products is commercially available, although the exact composition is in most cases unknown to the user. Figure 2.10 shows plastic blocks of four common embedding media, with different properties, as can be clearly seen from their color.

Their classification is based on the chemical structure of the reacting monomers. The main employed resin-classes are epoxide and methacrylate resins [53]. For reasons discussed later on, the melamine resins are to a less extent examined. The three classes are briefly introduced in the following.

Epoxide resins Because of their extraordinary resistance to the electron beam and the formation of extremely hard polymer blocks together with excellent cutting qualities, epoxide resin are the most widely-used embedding media for electron microscopic applications. Well-known examples are Epon812 [118], Durcupan [160], Quetol [109], Spurr [159] or Araldite [49, 48].

A simplified scheme of the reaction mechanism for the polymerization of epoxide resins is given in Fig. 2.11. One typical monomeric component is the epoxide, in most cases a glycidether or epichlorhydrine. The epoxide can be initialized to polymerize with various substrates: amines, amides, polyamides or dicarboxylic anhydrides [138]. All of these reactions are accelerated by heat. For biological samples the latter are used. These anhydrides, so called hardeners, carry long organic (aliphatic or also aromatic) side chains, which are decisive for the resulting polymer qualities.

The reaction mechanism between epoxides and anhydrides is quite complex. Simplified, the anhydride is forced to open by a trace of water or an alcoholic hydroxy group. Subsequently the half-ester thus formed reacts with the epoxide at the C-atom which carry the positive partial charge. However, by varying the $\mathrm{pH}$ of the reaction mixture, one can further tune the amount of ester- (basic conditions) over the amount of etherformation (acidic conditions).

The use of amines or amides as hardeners would result in epoxy-epoxy-reaction rather than copolymerization with the hardener. This would prohibit the assembly of the hardener side chains.

As mentioned previously, the constitution of these side chains is censorious for the texture of the polymer [119]. In general, more unpolar and longer residues give a softer polymer block than polarer or shorter ones. Moreover, if the side chains can at the reac- 


\section{Theoretical Background}

tive groups be further cross-linked with an epoxide or anhydride, this crosslinking would also result in a harder block. For Epon812 for example a high fraction of dodecenylsuccinicanhydride (DDSA) yields in a softer block than a high fraction of methylnadicanhydride (MNA).

In addition to the hardener, an accelerator like tri-(Dimethylaminoethyl-)phenol (DMP30 ) or dibutylphtalate is sometimes added. The latter also prevents the epoxide-components from crosslinking.

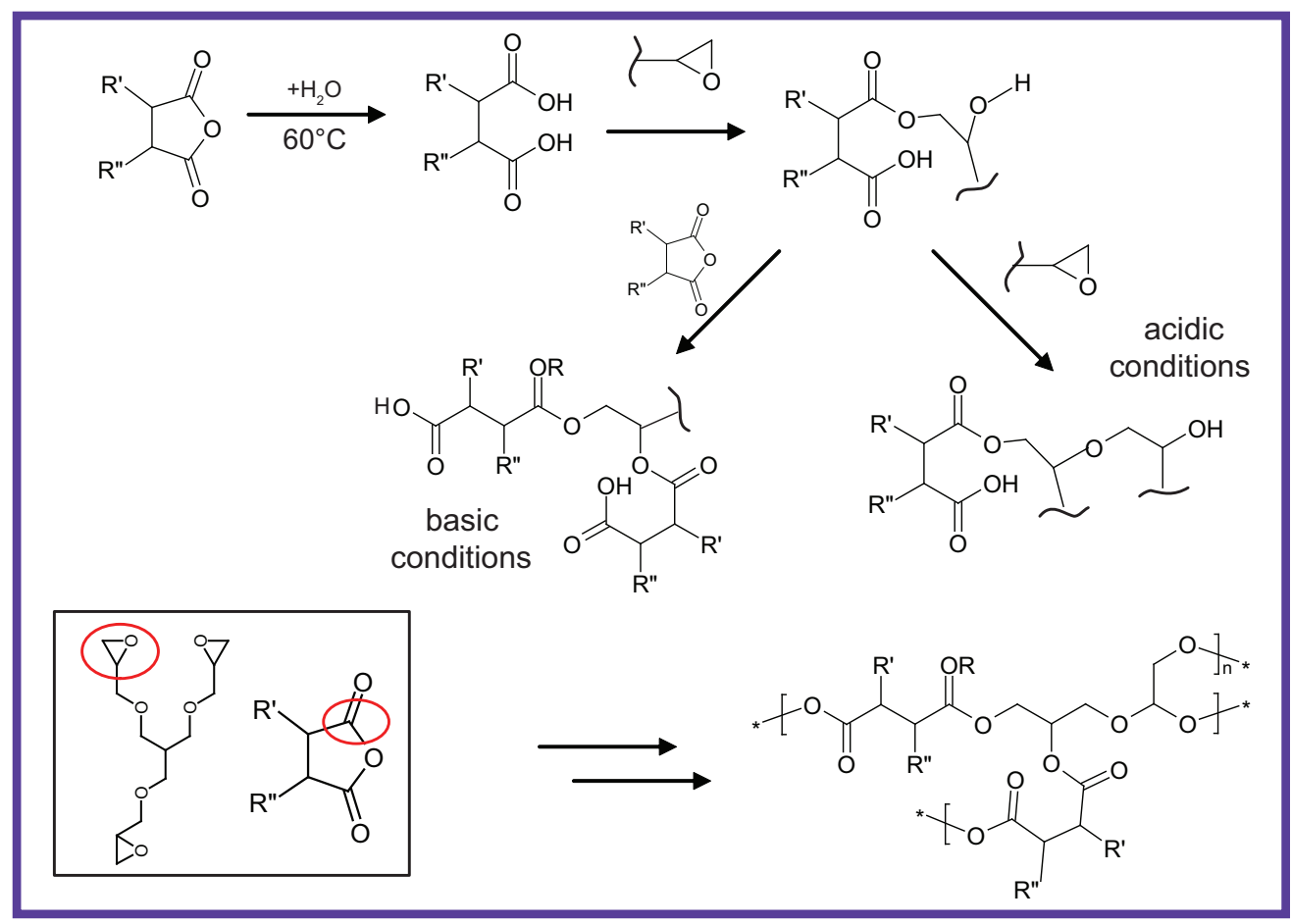

Figure 2.11: Simplified reaction mechanism for epoxide resins. The inset shows the main monomeric components, their reaction sites are indicated. R' and R" are organic side chains, with which the polymer qualities can be tuned.

The polymerization reaction already occurs at room temperature, but it is normally initialized by heat, implying one to four days at $60^{\circ} \mathrm{C}$. As oxygen would immediately stop the reaction, the polymerization must take place under exclusion of air in a tightly closed embedding tube. The same is true for water. The sample has to be dehydrated completely by a consecutive dilution series with an organic solvent like ethanol or acetone. Otherwise the anhydridic hardener would be deactivated which prevents the polymerization of the plastic.

Another drawback of epoxy resins is in most cases the high viscosity of the large monomer molecules, especially at the hardener site. This makes a homogeneous infiltration of the sample quite laborious and again necessitates a dilution series with organic solvents. 
Due to the high reactivity of the epoxide, the unpolymerized resins are in most cases toxic and should be handled with great care. Above all, several of the additives are known to be carcinogenic.

Acrylate resins Another prominent group of embedding media is represented by the acrylate resins, e.g. LR White [187], LR Gold [121], Microbed [146], Unicryl [146], Lowicryl [20]. Similar to the epoxide resins, they as well form a very hard polymer block, but normally show a higher grade of shrinkage, and therefore, a worse structure preservation. In addition, the acrylic resins are more sensitive to the electron beam.

The simplified reaction mechanism for the polymerization of acrylates is shown in Fig. 2.12. Most acrylic polymers used in biological applications are in fact methylmethacrylates. The reaction of the acrylate moiety is an addition at the C-C-double bond. This double bond reacts more readily than other C-C-double bonds, as the intermediate compound (a charge or a radical in the $\alpha$-position of the ester group) is stabilized by mesomerism. This explains, why other C-C-double bonds of cellular components are more inert.

The polymerization reaction is either initialized by heat $\left(50-60^{\circ} \mathrm{C}\right)$ or by microwave irradiation [79] or by radical starters. The latter sometimes require an activation by UV light [97]. In contrast, polymerization under basic conditions is for the benefit of the biological tissue not examined. However, the presence of oxygen, at least if no accelerator is present, is even more critical than it is for the epoxide resins. Even the wrong embedding mold might function as a scavenger and interrupt the radicalic polymerization reaction.

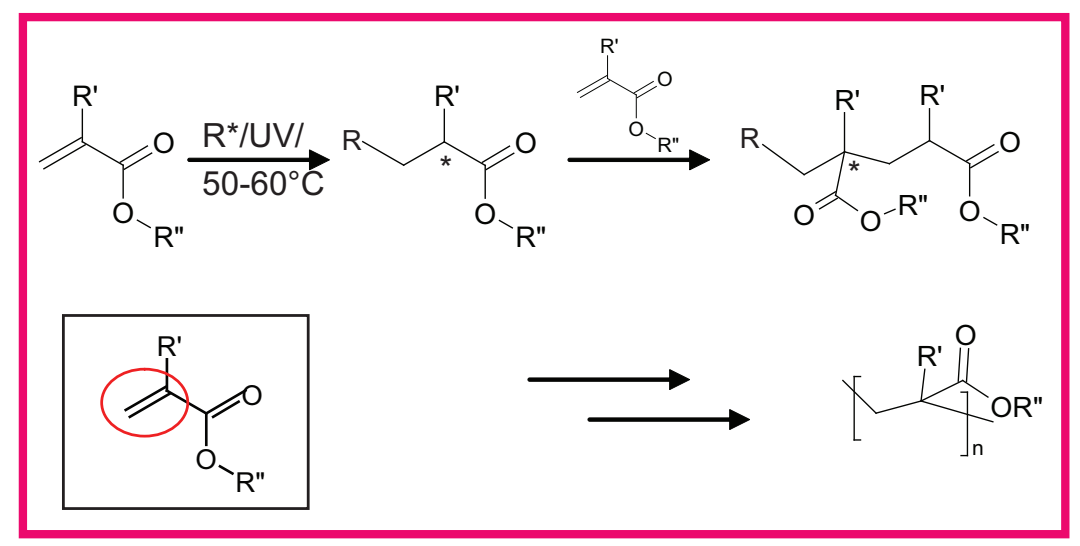

Figure 2.12: Reaction mechanism for the radical polymerization of acrylate resins. The intermediate state is stabilized by mesomerism with the adjacent ester moiety. The reactive double bond of the monomer is indicated in the inset. For methyl-methacrylates $\mathrm{R}^{\prime}$ is methyl.

The different acrylate resins can be distinguished by the side chains, especially at the ester function of the acrylate moiety. Furthermore, in some cases, additional cross linkers are added to tune the probabilities of the polymer in a desired way. This is similar to 


\section{Theoretical Background}

what has been described for the epoxide resins.

Also, the reaction conditions have an influence on the qualities of the resulting block. While heat polymerization damages most antigens and gives very hard blocks, chemically catalyzed polymerization at low temperatures or under UV light gives softer blocks.

Commonly the monomers of acrylate resins have a low viscosity, which is beneficial for the infiltration process. In contrast to the quite widely-used LR White, which already freezes at $-26^{\circ} \mathrm{C}$, Lowicryl resins stay handy even down to temperatures of $-80^{\circ} \mathrm{C}$. Clearly the compatibility with modern low temperature fixation and infiltration methods that gain more and more importance makes the acrylate resins indispensable.

Especially for softer blocks of acrylate resins, the antigenicity is better preserved compared to epoxide resin. On the one hand, this is because the antigens are easier accessible, if the structure is less crosslinked. On the other hand, antigens do not contain as many C-C-double bonds as they contain amino or hydroxy functions. therefore, they are less involved in the polymerization reaction of the acrylate resins.

Over and above, the acrylic resins sometimes allow a small amount of water (less than $30 \%$ ) during the polymerization reaction. This might not also protect lipophilic components from elution but is above all essential for preservation of proteinogenic fluorescence.

Besides, acrylic resins are in most cases transparent which make them more suitable for correlative approaches with light microscopy.

However, if they are polymerized by chemical activation, previous heavy-metal staining for electron microscopy would result in the production of extensive heat, which is highly destructive to the cellular structure.

Acrylic resins are not toxic, but only harmful. Anyway, contact of the liquid with skin and exposure to the vapors should be avoided to prevent allergenic reactions.

Melamine resins Melamine resins are very rarely used in sample preparation for electron microscopy, although they are highly resistant to the electron beam [186. The reason for this is obvious since the antigen preservation is very poor. Due to the mostly required postembedding staining, the latter is in electron-microscopic methods essential for protein specific investigations. The only melamine resin which has been reported with regard to biological studies is Nanoplast [5, 41].

The main monomeric component is a melamine, to be exact hexamethylol-melamineether (HMME), which is in the aqueous embedding solution partly hydrolyzed. Sometimes also a mixture of melamine itself with an aqueous solution of solid PFA is used. Melamine resins polymerize under poly-condensation and are normally used without co-polymerizers. There are also polymerization mixtures with further co-polymers available, but these have so far not been proven to be suitable for life-science application. The mechanism of the poly-condensation is simplified depicted in Fig. 2.13 .

The methylether functions, which are present in HMME or formed when melamine is employed with PFA, condensate under acidic conditions $(\sim \mathrm{pH} 4)$ at temperatures of $60^{\circ} \mathrm{C}$. This results in the formation of a polyether and a certain amount of water or methanol. Normally $p$-toluolsulfonic acid is used as the acidic catalyst. It does not form side products, as does for instance sulfuric acid. Increasing the $\mathrm{pH}$ yields softer blocks 
with less crosslinking, which are not suitable for ultrathin sectioning. However, decreasing the $\mathrm{pH}$ and inducing an all-over crosslinking at all ether-groups of the monomer makes the polymer brittle.

Certainly, amino- and hydroxy-groups of the biological tissue are also involved in the polymerization reaction. Thus the preservation of the antigenicity is even worse than it is for the epoxide resins. This destruction increases with the lowering of the $\mathrm{pH}$.

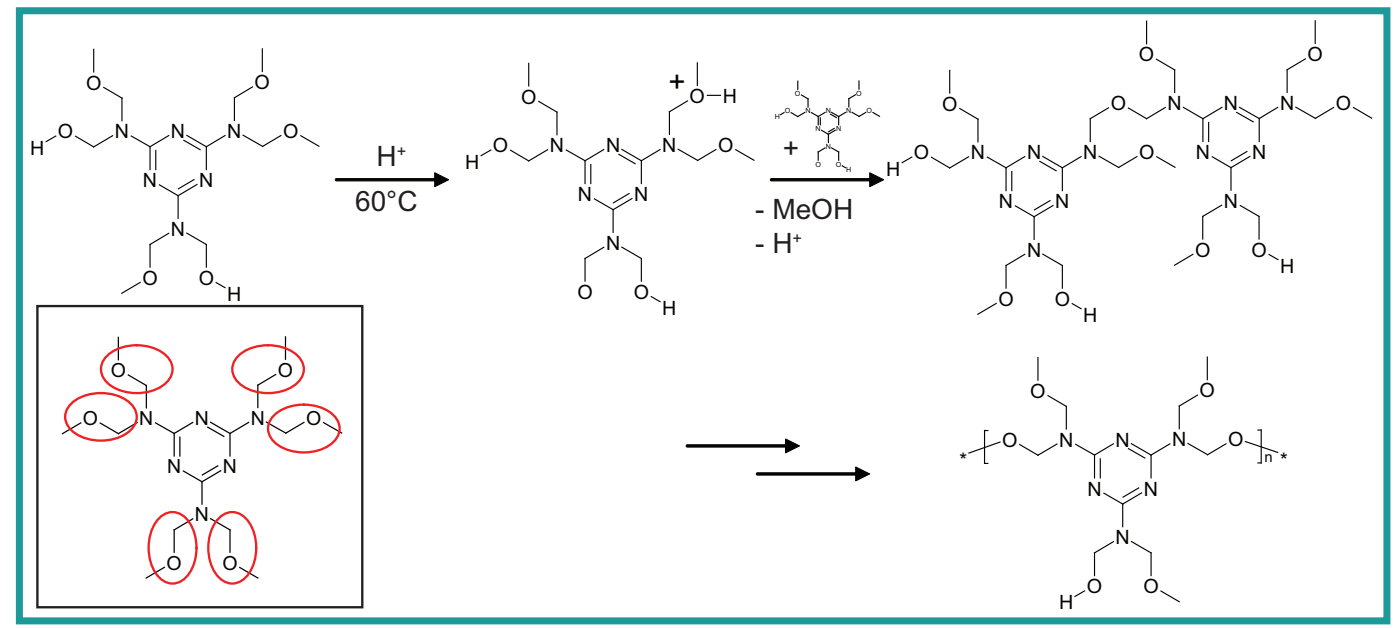

Figure 2.13: Simplified mechanism for the polymerization of HMME under acidic conditions. The methyl-ether is partly hydrolyzed in the aqueous reaction mixture.

As well as the antigenicity, the preservation of proteinogenic fluorescence is destroyed. This is because of the sensitivity of the chromophores to acidic conditions which induce isomerisation reactions.

Nevertheless, for various reasons, the transparent melamine resins should be taken into account as long as pre-embedding-staining is considered as an option. The monomers are completely soluble in water and a dehydration procedure, which is required for almost other resin classes, can be avoided completely.

However, the infiltration of biological samples lasts quite long, at least two days. In addition, before polymerization, the samples have to be dried over silica gel. This is performed at $40^{\circ} \mathrm{C}$ for at least one day. The time span increases with the size of the sample.

Still, melamine resins are comparably easy to handle because they are not sensitive to oxygen during polymerization and, even more important, not toxic or harmful. 



\section{Experiments and results}

\subsection{Embedding media for high-resolution light microscopy}

Since ultra-microtomy is a common technique for sample preparation in transmission electron microscopy, a number of embedding media, which in terms of their mechanical and chemical properties have been proven to be compatible with biological samples, are now commercially available. However, this does not necessarily mean that these are equally applicable for high-resolution fluorescence techniques. In fact, here the optical properties and their influence on the behavior of a fluorophore essentially govern their suitability.

Still, certain demands on the embedding medium are the same for high resolution fluorescence microscopy or electron microscopy. In terms of the mechanical properties, or in other words a good cutting performance, the polymer should show a homogeneous hardness. Brittleness has to be avoided to the benefit of a certain elasticity. Talking about the chemical properties of a resin, it should polymerize uniformly and without heavily affecting the fine structure of the sample. This includes that volume changes and shrinkage during the reaction are highly objectionable. What is furthermore conducive is a low viscosity of the monomeric compounds. Otherwise an even and rapid penetration into the tissue or any other structure is hardly ensured. The latter would be accompanied by irregular polymerization and bad sectioning qualities. Above all, especially for biological experiments, it is advisable that the monomeric compounds are soluble in water or other polar solvents to avoid as much elution of organic cell components as possible. Due to the concerted mechanical and chemical demands on an embedding medium for biological samples, it was advisable to resort to those which are already proven and well established in the field of electron microscopy.

However, the optical properties available embedding media for electron-microscopic application available embedding media are so far fairly unknown and thus will be outlined in this Chapter. This includes the investigation of the intrinsic auto-fluorescence at various excitation wavelengths and of the refractive index. Moreover, for several organic dyes, the influence of polymer-embedding on the fluorescence behavior is exemplarily tested.

Some other properties are more dependent on a certain experiment and thus not systematically evaluated. This is for example the resistance of the polymer against high laser power, which is comparable with the resistance against the electron beam in EM. The material's stability is substantially influenced by the section thickness, the section environment, and the laser wavelength. Several plastic media melt under the influence of the laser irradiation or even explode after absorption of the STED beam. Thus their suitability is highly dependent on the demands of a certain experiment. 


\section{Experiments and results}

Another issue is the bleaching characteristics of a dye in a certain environment. Beside the polymer type and the dye, further parameters like the cellular environment might have substantial influence on it. Hence, it is only rudimentarily tested in advance and with regard to a special application in detail in the appropriate Sections. This is also the case for further fluorescence staining techniques and their compatibility with resin embedding like staining with fluorescent proteins.

\subsubsection{Auto-fluorescence of the polymers}

Fluorescence microscopy on very tiny and often dim structures crucially depends on a large signal-to-noise-ratio(SNR). This holds even more for stochastic methods like PALM or STORM, which suffer more drastically from a background signal than STED does. Consequently, a strong auto-fluorescence of the embedding medium, which directly surrounds the structures, makes high-resolution imaging hard or even impossible. This auto-fluorescence might be caused by either the excitation or the STED laser beam, but in case of the latter it should be well cut off through appropriate filters. The autofluorescence properties of several embedding media at various excitation wavelength shall be investigated.

\section{Methods}

Fourteen commercially available embedding media were chosen and tested for their autofluorescence properties: One melamine resin (Nanoplast), six epoxide resins (Araldite, Durcupan, Epon812, Poly/Bed 812, Quetol 651, Spurr Low Viscosity (referred to as Spurr)) and seven acrylic resins (Lowicryl K4M, Lowicryl K11M, Lowicryl H20M, LR White, JB4, Unicryl, Microbed). To this end, each sample was polymerized between a cavity-carrying microscope slide and a coverslip according to the specified polymerization conditions. The detailed polymerization protocols are described in the Appendix. This ensured a constant thickness and deviations of less than $10 \mu \mathrm{m}$ of all samples except Nanoplast. Nanoplast, in contrast to all the others, depending on air contact for drying and polymerization, was prepared as a thin droplet on a coverslip. Consequently, Nanoplast layers were approximately twice as thick with a deviation of $50 \mu \mathrm{m}$. Polymer samples were put into a fluorescence spectrophotometer (Cary Eclipse Varian Inc., Palo Alto, CA, USA) and consecutively excited with light of three different wavelengths, $532 \mathrm{~nm}, 590 \mathrm{~nm}$ and $633 \mathrm{~nm}$ respectively. These wavelengths were chosen on the basis of common STED excitation wavelengths. The fluorescence was measured in the range of 100 or $110 \mathrm{~nm}$ above the excitation wavelength, where it is most distracting for STED imaging.

\section{Results and discussion}

Figure 3.1 shows the resulting fluorescence emission spectra. As the plots are color-coded for the different resin classes, one observation is at first glance prominent: Epoxide resins (plots shown in blue) show a comparably high fluorescence after excitation with any of the applied wavelength. In contrast, the melamine resin Nanoplast (green plot) and all 


\subsection{Embedding media for high-resolution light microscopy}

acrylate resins (magenta plots) show only a negligible signal. The off-peak signals at the beginning of the detection range of plot A (see inset in Fig. 3.1.1. A) are caused by scattered excitation light, which was not perfectly blocked by the applied filters.

The origin of the fluorescence observed for all epoxide resins is not easy to interpret. Raman scattering can be excluded to cause the signal, as small shifts in the excitation wavelength $(5 \mathrm{~nm}$ and $10 \mathrm{~nm}$ above and below the excitation light were tested respectively for each measurement) are not accompanied by the identical shifts of the emission maxima.

Furthermore, the fluorescence maxima of each resin alter after excitation with different wavelength. As Kasha's rule would otherwise be broken, there is no single transition between two distinct states which can be assigend to the auto-fluorescence of each resin.

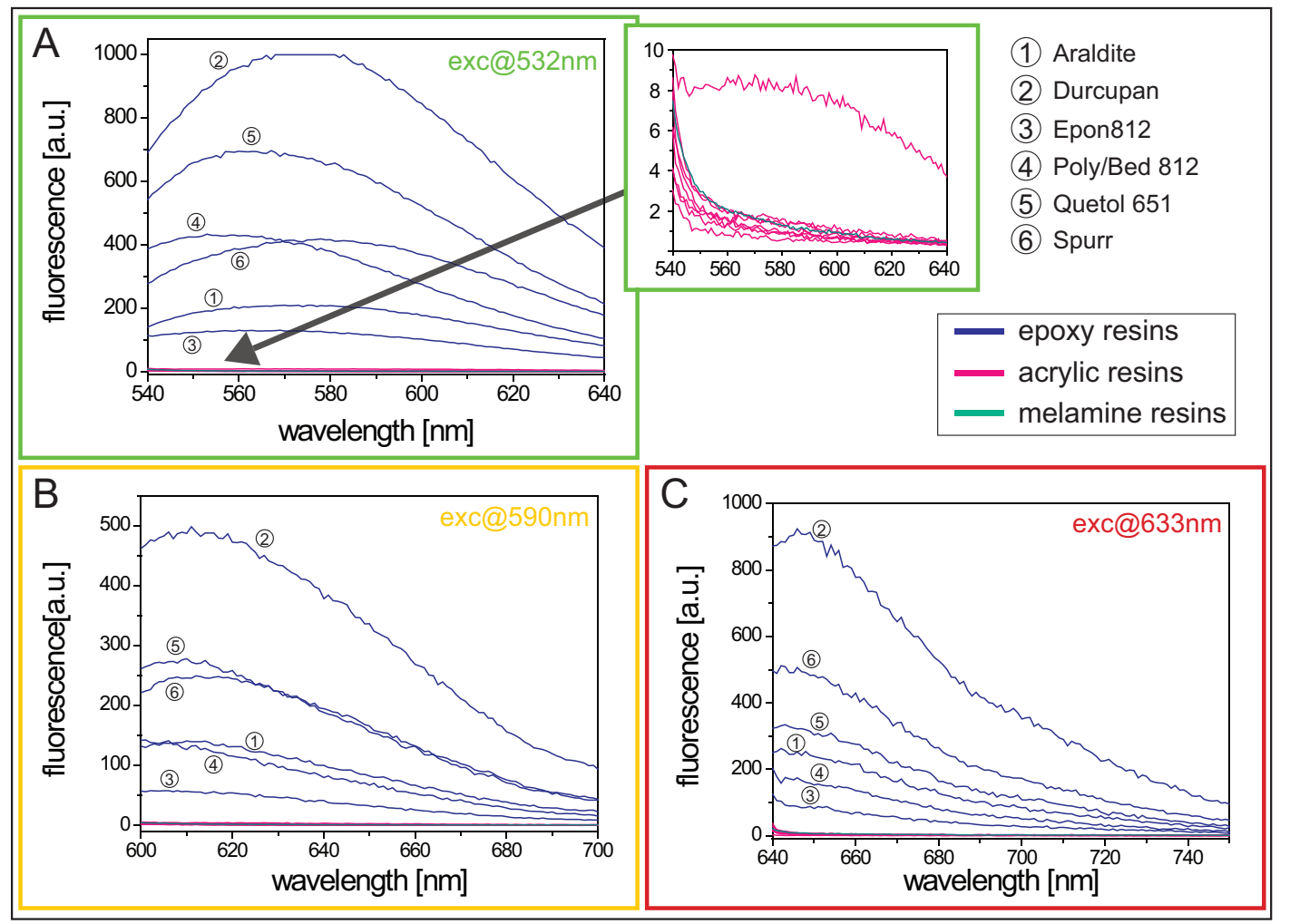

Figure 3.1: Auto-fluorescence spectra from different embedding-media. Excitation wavelength are $532 \mathrm{~nm}(\mathrm{~A}), 590 \mathrm{~nm}(\mathrm{~B})$ and $633 \mathrm{~nm}(\mathrm{C})$. The small green box shows an enlarged region of the fluorescence spectra at $532 \mathrm{~nm}$. Plot colors are chosen according to the different resin classes; blue: epoxide resins, magenta: acrylic resins, green: melamine resins.

However, based on this findings, it can be generally stated that acrylic or melamine resins are favorable in high-resolution fluorescence application. Anyway since in the 
final experiment only an ultrathin polymer section is supposed to be imaged, even the auto-fluorescence from the epoxide resins is likely to compete with the signal of a bright fluorescence label.

\subsubsection{Refractive indices of the polymers}

Microscope lenses are designed for a certain immersion medium with a specific refractive index, e.g. water $\left(n_{\mathrm{D}}^{20}=1.33\right)$, glycerol $\left(n_{\mathrm{D}}^{20}=1.474\right)$ or immersion oil $\left(n_{\mathrm{D}}^{20}=1.518\right)$. Any deviation from this intended refractive index introduces optical aberrations which become more pronounced as the thickness of the mismatching medium increases. As a result, the focusing performance suffers and with that the resolution capabiliity of the system. While glass coverslips, which are normally used as substrates for the biological samples, are already considered in the design of the objective lens, the within the sample applied mounting medium should be adapted in terms of the refractive index. An investigation of a sample in aqueous solution for example should ideally be performed with a water objective lens. In contrast, for an oil objective lens, mowiol $n_{\mathrm{D}}^{23}=1.49$ or $97 \% 2,2^{\prime}$ thiodiethanol (TDE) $n_{\mathrm{D}}^{23}=1.515$ should be favored as mounting media [161].

However, resin sections are quite compact and thus hardly penetrated by any mounting medium. Therefore it is desirable that the resin itself matches the refractive index of the objective. To this end, the values of $n_{\mathrm{D}}^{20}$ are determined for the fourteen different embedding media already used in the previous Section. Still, one should keep in mind that the final aim is the investigation of ultrathin sections. Hence a certain mismatch in the refractive index of a resin might be tolerable in the end. Moreover, the aberrational effects which might occur can to a certain extent be corrected by subsequent calculations.

\section{Methods}

The fourteen embedding media were polymerized in thin layers of several hundred micrometers thickness between Aclar film. Subsequently, they were investigated with an Abbe refractometer using $\alpha$-bromo-naphthalene as contact liquid to avoid a layer of air in between the apparatus and the sample.

\section{Results and discussion}

Figure 3.2 shows the resulting values for the refractive indices of the different polymers. All of them are quite high, so that oil objectives, which have the highest value for $n_{\mathrm{D}}^{20}$ among the commonly applied objective lenses, are to be favored for the investigations of ultrathin polymer sections .

Except for the epoxide resin Durcupan, with an $n_{\mathrm{D}}^{20}$ of 1.654 , more or less all of them were in a reasonable range for this purpose. Polybed, which is also an epoxide resin, even exactly matches the value of immersion oil and would thus be favorable in this respect. In contrast, the values for Araldite or the melamine resin Nanoplast diverge quite a lot and are therefore marginal with regard to the application in optical high resolution methods.

However, while for acrylate resins the values are quite consistent, those for the epoxide resins are distributed over a larger range. Thus it is hard to predict the value of $n_{\mathrm{D}}^{20}$ for 


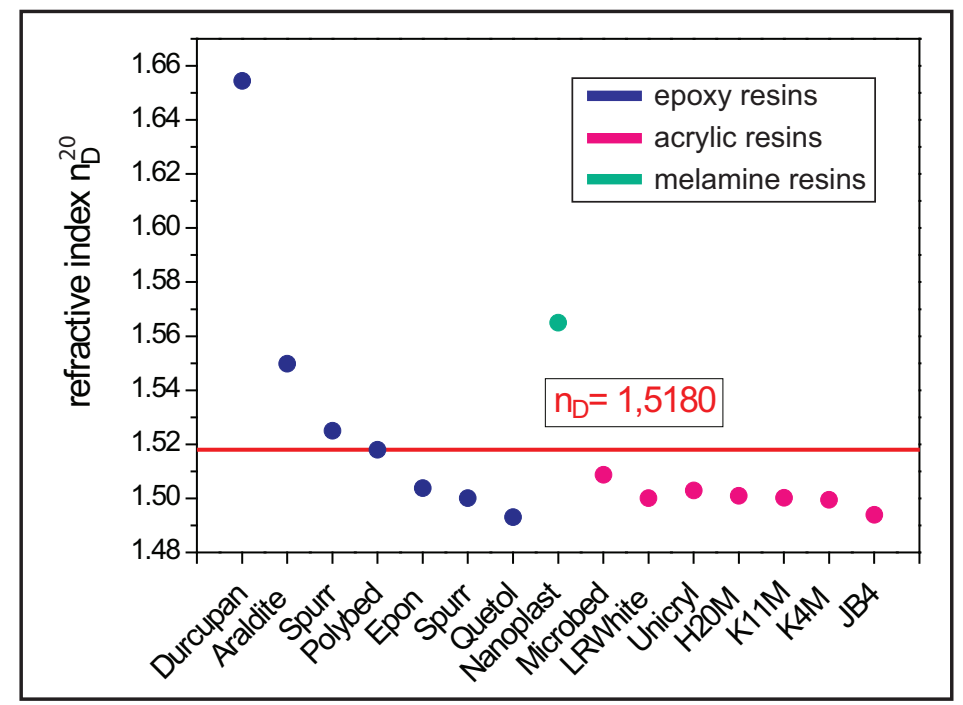

Figure 3.2: Refractive indices of different embedding media. Spot colors indicate the resin class of an individual substrate; blue: epoxide resin, magenta: acrylic resins, green: melamine resins. For reference the refractive index of glass is indicated by the red line.

a class of a certain resin. In any case, the in here described results have to be associated to further properties of a certain resin if its suitability for optical microscopy is to be rated.

\subsubsection{Properties of fluorescent dyes in polymers}

While working with fluorescence microscopy and STED microscopy in particular, one is in most cases reliant on labeling the structure of interest with a fluorophore. In most cases these are synthetic organic dyes. The most common way for selected labeling of proteins is immuno-staining with specific antibodies which are on their part recognized by secondary antibodies carrying one or several fluorophores. In electron microscopy small metal particles, like silver or gold, are used as visible markers. As both the tissue penetration and the cellular uptake of such toxic and comparably heavy particles are hindered, a post-embedding staining of the single ultrathin sections is generally the method of choice. Consequently, the preservation of proteinogenic epitopes during the polymerization process of the embedding medium is essential for a subsequent recognition by antibodies.

For fluorescence immuno-staining this is not compulsory, although sometimes advantageous, when bulk tightly packed structures are not approachable by the voluminous antibodies. In most cases, a much more efficient and unspoiled immuno-labeling can be achieved by pre-embedding staining. This is in particular important for high resolution methods where labeling artifacts are more likely to be revealed. In the fixed, but in other 


\section{Experiments and results}

respect intact cell, a chemical masking or damaging of the epitopes by the polymer is excluded and the epitopes have not been physically truncated by the sectioning itself. Particularly for high-resolution imaging, where signals from single molecules can be read out, such an effective labeling of the whole structure is essential. This on the other hand requires the fact that not so much the epitopes, but the fluorescence of the marker is left unaffected by the hardening process. Hence, an important issue is the compatibility of the polymer and its hardening reaction with either the staining method or the herein used fluorophore. Moreover, the changed environment of the fluorophore in a polymer might either quench the fluorescence to a certain extent or cause shifts in the absorption and emission spectra. Finally, variations in the fluorescence lifetime of a dye, especially a reduction, are critical for STED imaging because this hinders an effective fluorescence depletion by the STED beam.

The use of fluorescent proteins for labeling is in many cases an attractive alternative. However, since there are only a few suitable ones for STED microscopy and as they are especially sensitive to the embedding procedure, they are excluded from a general screening at this point. Further staining methods are also not the subject of this study. The less specific interactions of lipophilic membrane dyes or DNA intercalators are stable in aqueous solution but easily disturbed by environmental changes that happen during the infiltration procedure. The fact that not only the optical properties of the dye are affected but also the specificity of the staining forecloses a general examination.

Consequently, the following Sub-section deals with the altered properties of organic dyes after polymer embedding. In addition to the preservation of the fluorescence of ten dyes, changes in the emission spectra of two different fluorophores, ATTO647N and ATTO532, after polymer embedding are exemplarily discussed. Subsequently, the sensitivity of the fluorescence lifetime of ATTO532 under different environments is also briefly addressed.

\section{Fluorescence preservation}

\section{Methods}

Ten fluorescent organic dyes of different structural classes, which had been to a greater or lesser extent successfully used for antibody staining and subsequent STED imaging, were selected. These are ATTO532 (rhodamine derivative), Oregon Green488 (rhodamine derivative), ATTO647N (carbopyronin derivative), Cy3 (cyanine derivative), 7-Diethylamino-Coumarin (coumarin derivative), Dyomics V07-02060 (structure not published), EosinY (rhodamine derivative), MR121 (oxazin derivative), Bodipy (boradiazaindacene derivative) and Squaraine Rotaxane 3 (squarain derived rotaxane). A $1 \mu \mathrm{M}$ solution of the dyes in DMSO was diluted in $1 \mathrm{ml}$ of the monomer mixture of each of the fourteen different embedding media mentioned previously. All resins were thermally polymerized. As described in Part 3.1.1 for the pure resins, the samples were polymerized between cavitycarrying microscope slides and coverslips. Subsequently the fluorescence spectra for the appropriate excitation wavelength and appropriate emission range (emission maximum in water $-10 \mathrm{~nm}$ to emission maximum in water $+200 \mathrm{~nm}$ ) of the single dyes were taken. 


\subsection{Embedding media for high-resolution light microscopy}

The step size was chosen to be $1 \mathrm{~nm}$ with a $5 \mathrm{~nm}$ wide excitation and emission slit, a medium detector voltage and a dwell time of $0.1 \mathrm{~s}$.

\section{Results and discussion}

Table (3.3) shows whether the fluorescence of a dye was successfully preserved in an embedding medium or not. This rating was made according to Roose's criterion [135]: whenever the in the fluorescence spectrometer detected signal-to-noise-ratio $\geq 5$, the corresponding cell of the table was marked with "+", otherwise with "-". The values were determined from the maximum and minimum values respectively detected within the emission spectra. For better visualization, the number of dyes which maintained their fluorescence in a certain embedding medium is shown in a bar plot.

\begin{tabular}{|l|l|l|l|l|l|l|l|l|l|l|l|l|l|l|}
\hline Atto532 & - & - & + & - & + & - & + & - & + & + & + & + & + & + \\
\hline OregonGreen & - & - & - & - & - & - & + & - & - & - & + & - & - & - \\
\hline Atto647N & - & - & - & - & + & - & + & - & + & - & - & + & - & - \\
\hline Cy3 & - & - & + & - & - & - & + & - & + & - & + & + & + & + \\
\hline Coumarin & - & - & + & - & + & - & + & - & + & - & + & + & + & - \\
\hline DyomicsV07 & - & - & - & - & - & - & + & - & - & - & - & - & + & + \\
\hline EosinY & - & - & - & - & - & - & + & - & - & - & + & - & - & - \\
\hline MR121 & - & - & - & - & - & - & + & - & - & - & - & - & - & - \\
\hline Bodipy & - & - & - & - & - & - & + & - & - & - & - & - & - & - \\
\hline Squaraine & - & - & - & - & - & - & + & - & - & - & - & - & + & - \\
\hline & \\
\hline
\end{tabular}

Figure 3.3: Table of different STED dyes plotted against different embedding media. Whenever the SNR was observed to be $\geq 5$ at the emission maximum of the dye, it is assigned "+", otherwise a "-". Marks are color-coded according to the different resin classes; blue: epoxide resins, green: melamine resins, magenta: acrylic resins. A bargraph quantifies the number of detectable dyes in each resin.

The most remarkable observation is that Nanoplast is the only resin in which all of the dyes maintained a prominent characteristic fluorescence spectrum. Also, $\sim 40 \%$ 


\section{Experiments and results}

of the dyes embedded in acrylic resins remained fluorescent, while in epoxide resin the fluorescence is rarely detectable. The Nanoplast samples were twice as thick compared to all the other resins (see Part 3.1.1). This would explain a roughly twofold enhanced fluorescence signal of all dyes. Still, the signals in Nanoplast were even more than twice as high, so that the difference in the sample preparation can not exclusively be responsible for the observations.

Several issues may contribute to the loss of fluorescence in epoxide and acrylic resins. First, for the highly auto-fluorescent epoxide resins, the characteristic emission spectra of the dyes might be overlapped by the background signal and is thus not detectable anymore (compare Part 3.1.1). Second, the embedding medium might quench the fluorescence signal of the dyes. The absorbed energy is released to the polymer as thermal energy rather than under emission of a fluorescence photon. Third, the fluorescent dye might have been partly or completely destroyed during the polymerization reaction. Although the polymerization conditions were chosen to be as mild as possible, the chromophoric $\pi$-electron system of a dye might have been affected. The application of UV light was completely omitted, since this might have caused photo-bleaching of the dyes.

Which mechanism finally caused the loss of fluorescence in a specific case is not easily accessible. But due to the lacking relevance in this context, it was not made a subject of further investigations.

Spectral shifts after resin embedding Even if a preservation of the fluorescence is ensured for a certain dye, compared to an aqueous solution, shifts in its emission spectrum might occur due to the environmental changes in a polymer. In fluorescence techniques this could require an according adaption at the setup, e.g. a replacement of the fluorescence filters or, with respect to STED microscopy, a shift of the wavelength of the STED beam.

\section{Methods}

To exemplarily test the changes in the fluorescence behavior of a dye after embedding in different embedding media, the fluorescence spectra of two dyes, ATTO532 and ATTO647N, were measured. The preparation of the samples and the parameters of the measurement were identical to those in a previous section (Part 3.1.1).

\section{Results and discussion}

The resulting fluorescence spectra of ATTO532 and ATTO647N are shown in Fig. 3.4. As reference the spectra of their aqueous solution are also plotted in black color.

For ATTO532 the fluorescence maximum $\lambda_{\max }$ in water is at $552 \mathrm{~nm}$, but this is shifted after polymer embedding. It is remarkable that in epoxide resins the fluorescence maximum is persistently moved to longer wavelengths, whereas the largest shift occurred for Quetol. In case of the latter, the maximum was at $570 \mathrm{~nm}$. This is similar to the behavior of the dye in less polar solvents like DMSO. Here, the fluorescence maximum is found to be at $565 \mathrm{~nm}$ (spectrum not shown). As epoxide resins are known to be hydrophobic, 


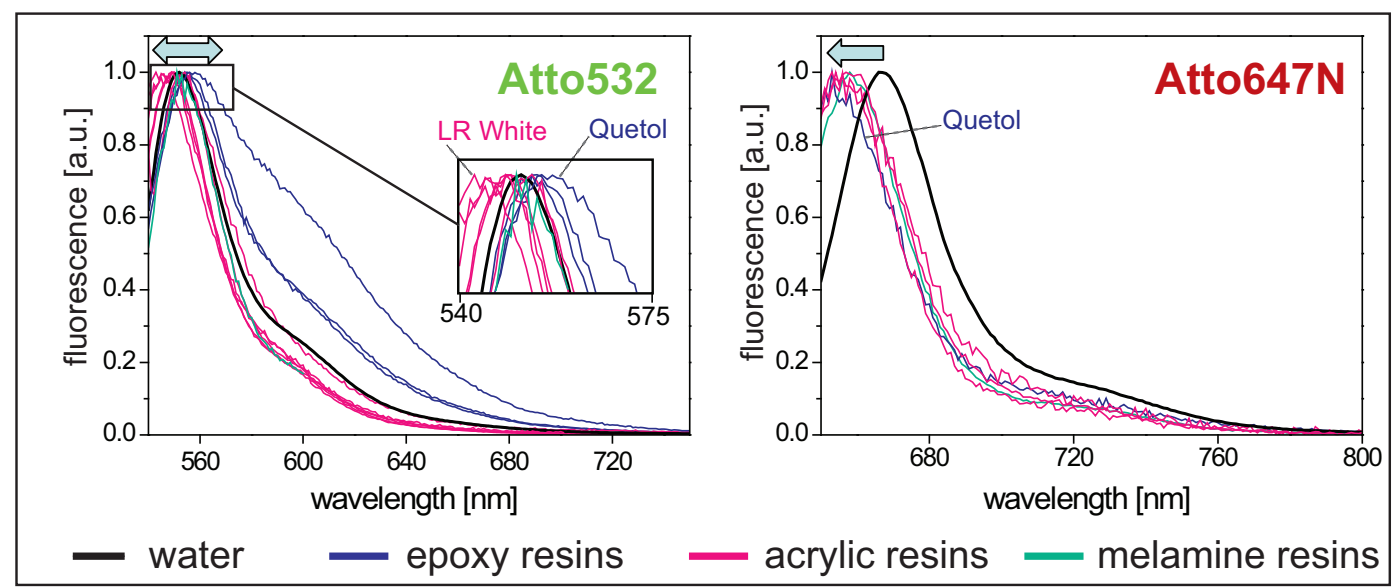

Figure 3.4: Fluorescence spectra of two selected dyes embedded in different resins. Plot colors indicate the according environment, the spectra was measured in; blue: epoxide resins, magenta: acrylic resins, green: melamine resins, black: reference spectrum in water.

the bathochrome shift might in both cases be due to a stabilization of the $S_{1}$ state of the dye in an unpolar environment.

In contrast, for the less hydrophobic acrylic resins, the spectra are slightly shifted with regard to the spectrum in water. Sometimes even a small blue shift occurs. This is most distinct for LR White where $\lambda_{\max }$ is found to be at $545 \mathrm{~nm}$, accordant to a shift of $7 \mathrm{~nm}$. Either a stabilization of the $S_{0}$ state or a destabilization of the $S_{1}$ state could be responsible for this. Interestingly, the spectrum in Nanoplast is more or less identical to that in water.

For ATTO647N it is slightly different. While the emission maximum in water is assigned to $669 \mathrm{~nm}$, it is shifted to lower wavelength in all polymers. A stabilization of the excited $S_{1}$ thus does not occur. In contrast, either the $S_{1}$ state is destabilized or the $S_{0}$ state is stabilized. This seems to be even more the case the less polar the environment. Therefore, the fluorescence maxima in epoxide resins differ more from the one in water, than for acrylate or melamine resins. This is supported by the observations in unpolar solvents. For DMSO, the shift is negligible. For the even less polar acetonitrile, a small hypsochrome shift of $5 \mathrm{~nm}\left(\lambda_{\max }=664 \mathrm{~nm}\right)$ is observed. The most pronounced shift is again found in the epoxide resin Quetol with $17 \mathrm{~nm}$.

The obtained results show that the spectral behavior of a dye is influenced by a certain polymer environment, but not dramatically altered. Most of the observations are conform within a certain resin class for a certain dye. In contrast, a transfer of the results for a certain dye to it's whole structutral class is hardly possible. Variations in the side groups might rather cause significant changes in terms of molecular interaction with the polymer.

With respect to STED microscopy, the shifts of the fluorescence emission maxima are tolerable and will most likely not influence the successful application of any polymer em- 


\section{Experiments and results}

bedded dye. Varying the wavelength of the STED beam might circumstantially improve the resolution. Still, this has to be tested individually in each application because of further influences by the local (cellular) environment.

Variations in the fluorescence lifetime after embedding With respect to application in STED microscopy, the fluorescence lifetime of a dye is quite important. The shorter the lifetime the less effective is the stimulated deexcitation from the $S_{1}$ state by the STED beam. Instead, more fluorophores have under the emission of a fluorescence photon already returned to the ground state when the STED pulse arrives. Thus it has to be tested what kind of influence a polymer embedding has on the fluorescence lifetime of a dye.

\section{Methods}

To investigate the variation of the fluorescence lifetime of a dye, in this case ATTO532, six different samples were prepared. A $1 \mu \mathrm{M}$ solution of the dye was prepared in the pre-embedding solution of either Nanoplast or LR White and in water. The monomer solutions were subsequently polymerized, in case of LR White in a covered cavity-carrying slide, in case of Nanoplast as a droplet on a coverslip. Additionally three samples of PtK cells cultured on coverslips were immunologically stained for $\beta$-tubulin. These samples were accordingly embedded in LR White and Nanoplast respectively, or left in water. The samples were investigated with a setup described elsewhere [46]. Briefly, the excitation of the fluorophore was performed with a pulsed $80 \mathrm{MHz}$ laser diode at $532 \mathrm{~nm}$ (PicoTA from Picoquant, Germany) and the fluorescence decay was subsequently detected with a single photon counting card (SPC730 Becker \& Hickel, Germany) at five different locations on each sample. The subsequent data analysis to obtain the fluorescence lifetime was performed with the software LabVIEW (National instruments, Austin, Texas, USA).

\section{Results and discussion}

The resulting fluorescence lifetimes are visualized as a bargraph in Fig. 3.5. Generally, the observed fluorescence lifetimes of ATTO532 vary substantially for the pure dye compared to the case, when it is via immuno-staining attached to a cellular structure (in this case $\beta$-tubulin). In a direct cellular environment the values for $\tau_{\mathrm{fl}}$ are more than 2 ns smaller in each case. This is true for the samples in aqueous solution as well as in a polymer.

However, if one compares the fluorescence lifetimes in aqueous solutions with those in either LR White or Nanoplast, the fluorescence lifetimes are only slightly larger in the latter cases (about $0.3 \mathrm{~ns}$ ). This observations are conform for the pure dye as well as for those where the dye was previously attached to $\beta$-tubulin and is irrespective of the polymer.

In any case, the values fit the applied laser pulse ranges of a typical STED experiment. As STED imaging has been successfully performed with ATTO532 in aqueous solutions, a perturbing influence of the polymer embedding in one or the other resin is unlikely. 
Instead, the slightly longer lifetimes of the dye should induce an even better performance after resin embedding.

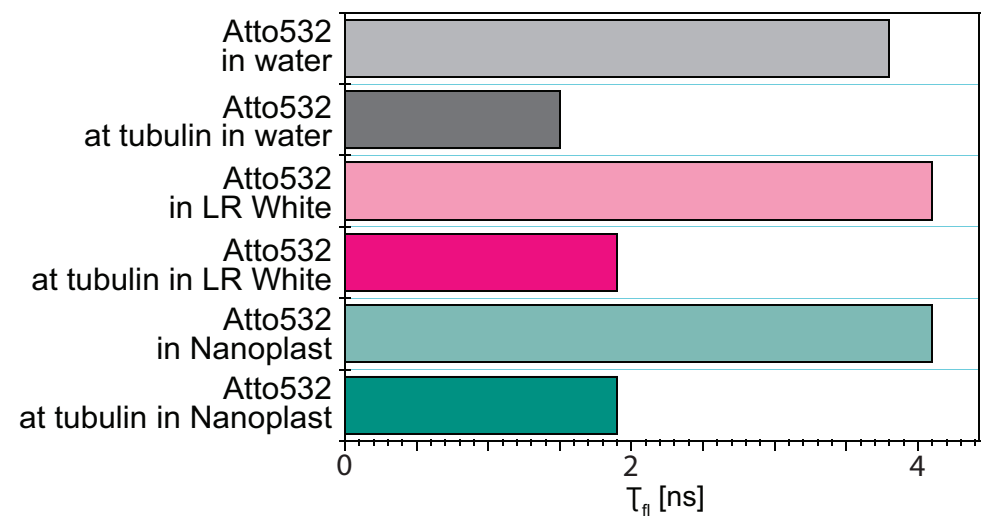

Figure 3.5: Fluorescence lifetime of ATTO532 in different environments: Water (light gray), LR White (light magenta), Nanoplast (light green). The same measurements were also performed with the dye attached to a cellular structure (tubulin) in water (gray), LR White (magenta) and Nanoplast (green).

\section{Photo-bleaching behavior after resin embedding}

\section{Methods}

To test the influence of triplet quenchers on the bleaching behavior of fluorescent dyes in Nanoplast, three different substances (mercaptoethylamine (MEA), [1,4]-diazabicyclo[2.2.2]-octane (DABCO), n-phenylglycine (NPG)) were co-embedded in Nanoplast with ATTO647N at various concentrations $(1 \mathrm{mM}, 0.1 \mathrm{mM}, 0.01 \mathrm{mM})$. The concentration of ATTO647N was according to previous experiments $1 \mu \mathrm{M}$. In addition, a $1 \mu \mathrm{M}$ of the pure dye and a $1 \mu \mathrm{M}$ solution of ATTO647N covalently coupled to BSA were investigated. Basically, the samples were prepared as described previously (see Section 3.1.1). Each of the samples was investigated in a STED setup in detail described at the beginning of the subsequent Chapter. An area of $3 \mu \mathrm{m} \times 3 \mu \mathrm{m}$ was scanned ten times with an excitation beam power of $4.5 \mu \mathrm{W}$. After each scan, another scan was performed with the combination of both the excitation and the STED beams, while the STED power was stepwise increased from $5 \mathrm{~mW}$ to $50 \mathrm{~mW}$. The pixel size was chosen to be $100 \mathrm{~nm}$, the according pixel dwell time was $50 \mu \mathrm{s}$. Afterwards the average fluorescence obtained from the first and the last excitation scan were compared.

\section{Results and discussion}

As can be seen from the bargraph in Fig. 3.6, the bleaching rate of ATTO647N in Nanoplast without any additives is quite high. After ten subsequent scans, only about $20 \%$ of the fluorescence signal was left. The observed signal could be about twofold 


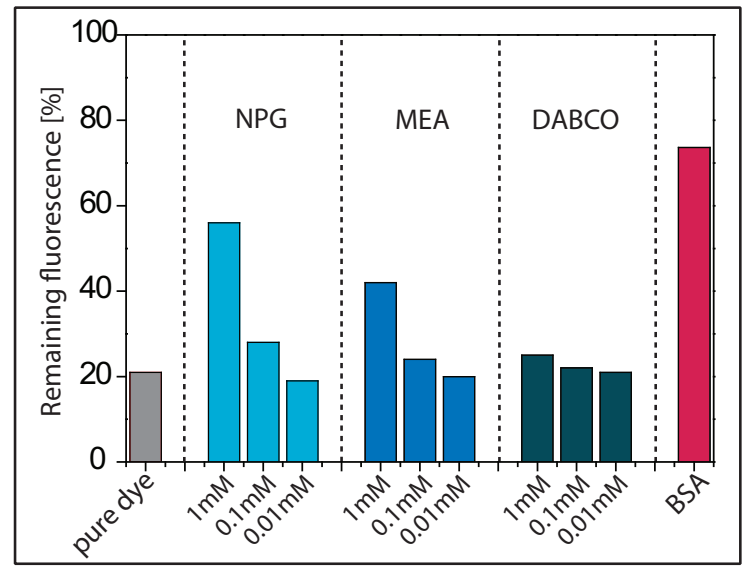

Figure 3.6: Fraction of the initial fluorescence observed after ten scnas on samples of ATTO647N in Nanoplast. The pure dye (gray) is compared to the dye in the presence of three triplet quenchers at various concentrations (blue) and the dye covalently linked to BSA (magenta).

improved by the co-embedding of the triplet quenchers NPG and MEA in quite high concentrations $(1 \mathrm{mM})$. In contrast, lower concentrations were in either case hardly effective. For DABCO, which is another triplet quencher, no effect was observed.

However, the most promising results were obtained from the sample where the dye is directly attached to BSA, which can be interpreted as a representative of cellular structure. The fluorescence signal dropped only by about $25 \%$ after ten subsequent scans. Since the initial fluorescence intensity was about the same as for all the other samples, this was not caused by a higher concentration of the dye.

Consequently, the photo-bleaching which is observed in a pure solution of the dye in Nanoplast is much more effectively reduced in the proximity of the cellular environment than by the co-embedding of additional anti-fading reagents. For the latter comparably high concentrations would be necessary to observe the same resistance against photo-bleaching. These are required to ensure that the fluorophore and the co-embedded quencher are close enough to result in the desired effect. Hence, in future experiments, where exclusively biological structures are investigated, the addition of triplet quenchers to the resin solution was abandoned.

\subsubsection{Conclusion}

Based on the results presented in this Chapter no resin could be irrevocably excluded from the application in high resolution light microscopy on ultrathin sections. However, Nanoplast seems to have some advantageous qualities over other resins. It shows an extremely low auto-fluorescence signal at different excitaiton wavelengths and moreover does not affect the signal of all tested organic fluorophores. A minor drawback is the quite high refractive index of the resin. But as the section thickness in the final experiment is 
supposed to be only a fraction of the wavelength, this can be assumed to be negligible.

Still, ultrathin sections of either any acrylate or epoxide resin should not completely be foreclosed from the application in optical high resolution methods. Due to their good sectioning qualities or due to the labeling requirements of a certain experiment, it is in some cases necessary to consider them. Again, the aim is the investigation of ultrathin sections. Thus, for example, the auto-fluorescence signal from such a thin layer is highly reduced and reasonably low compared to that from a bright fluorescent label. In addition, one should always bear in mind that post-embedding labeling is always an alternative, if the fluorescence of a dye is destroyed by the embedding process.

In any case, it is mandatory to directly test the perfomance of a dye in a certain STED experiment. The direct environment, on which the cellular and the polymer structures have a share, inpredictably influences its specific behavior. Especially the shifts in the fluorescence maxima, but also the bleaching behavior need to be questioned on an individual basis. 


\subsection{STED nanoscopy on ultrathin sections}

In this chapter, high-resolution imaging on ultrathin sections is shown exemplary on different samples. To validate the principle of STED on an immuno-stained, resin embedded and ultrathin sectioned sample, the fluorescence depletion efficiency as a function of the applied STED power is examined at first. Afterwards, the resolution capacity itself is verified on STED images of biological structures using different STED setups working with different fluorescent dyes. Moreover, the achieved resolution with resin embedded and ultrathin sectioned samples is compared to the corresponding bulk samples to illustrate the beneficial effect of the additionally implemented subdiffractional resolution in the axial direction. Finally, the capacity of the described mechanical approach is compared with isoSTED microscopy, an all-optical approach to three-dimensional nanoscale imaging.

For all experiments described in this Chapter, Nanoplast was applied as the polymer resin. According to the outcome of the experiments described in Chapter 3.1, successful high-resolution imaging is most likely achieved by using this plastic, not least since the properties of fluorescent dyes are retained best in this medium.

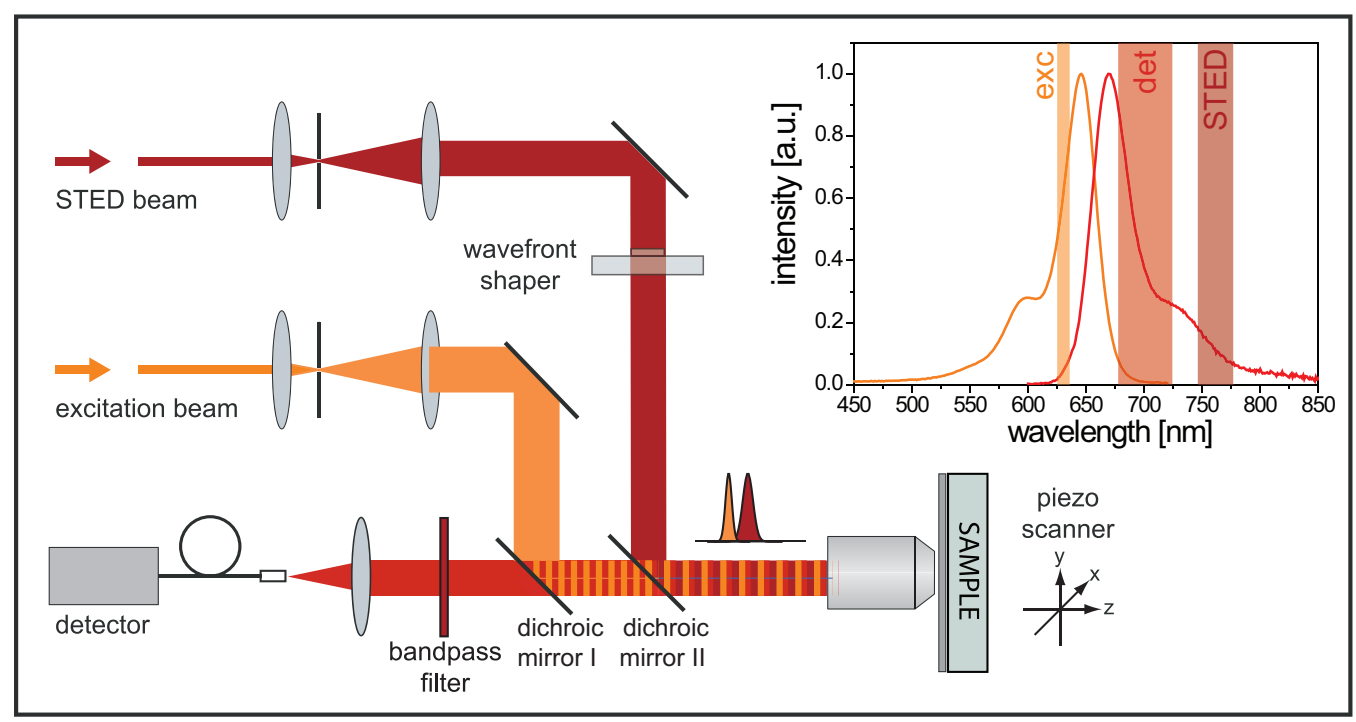

Figure 3.7: Schematic of a typical STED setup. The excitation and the pre-shaped STED beam are superimposed and focused into the sample while being separated from the detection light by dichroic mirrors. The two laser pulses are synchronized and timed such that an excitation pulse is directly followed by a STED pulse. The fluorescence is cleaned up by a bandpass filter and guided to the detector, usually an avalanche photo diode. Stage scanning is performed by moving the sample relative to the focus using a piezo scanner. The inset shows a typical absorption and fluorescence spectrum of a dye, along with the wavelength ranges for excitation, STED and detection light. 


\subsubsection{Fluorescence inhibition on ultrathin sections}

The principle of STED relies on the fact that a certain fraction of molecules, which were initially excited by an excitation laser, can effectively be forced back into the nonfluorescent ground state by inducing stimulated emission through the STED laser. Consequently, assuming a donut-shaped PSF of the STED beam, only those molecules in close proximity to the excitation center contribute to the detected fluorescence signal. Therefore the focal volume of the effective PSF is reduced, subdiffraction imaging is enabled. However, the indispensable condition for this mechanism is the fact that the STED laser can efficiently turn excited fluorophores back into their non-fluorescent state. The depletion factor $\eta$ quantifies the probability of an excited molecule to fluoresce as a function of the STED intensity. As the latter is increased, $\eta$ converges towards zero. The typical behavior of $\eta$ is in this Section exemplarily shown for ATTO647N.

\section{Methods}

The immuno-stained samples were prepared as described in the Appendix.

A typical STED setup is shown in Fig.3.7. The excitation and detection paths are identical to those of a confocal setup. In this particular case ATTO647N has successfully performed as the working-horse for this setup [58]. For ATTO647N, whose absorption and emission spectra are shown together with the described laser wavelength in Fig. 3.7, inset, a pulsed laser diode with $\lambda_{\text {exc }}=635 \mathrm{~nm}$ at a pulse length of $70 \mathrm{ps}$ is used. The respective STED beam is generated by a Titanium-Sapphire laser at a flexible wavelength, typically $\lambda_{\mathrm{STED}}=760 \mathrm{~nm}$ for ATTO647N. Its pulse length is stretched to $\sim 300 \mathrm{ps}$ (100 fs initially) by dispersion in a polarization maintaining single mode fiber. The pulse is prestretched by guiding the light first through about $1 \mathrm{~m}$ of glass rods (SF6) in order to avoid nonlinear effects in the fiber. To overlay the two laser pulses in time, a photodiode within the STED laser serves as the trigger. The excitation pulse can be adjusted to the STED pulse in time with an accuracy of approximately 10 ps by a delay unit.

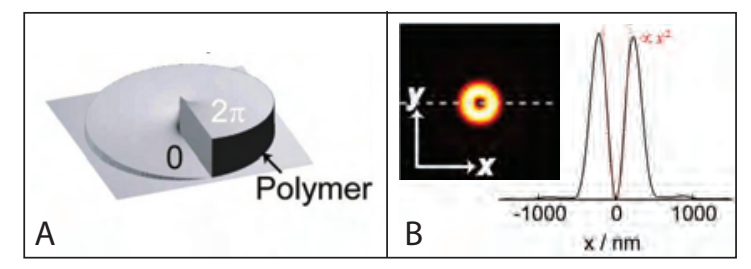

Figure 3.8: A: Profile of a polymer phase mask designed for beam shaping. B: Image and line profile of a toroidal STED beam, in this case generated by a phase mask.

To generate the most efficient imaging mode, a donut-shaped STED beam with an intensity zero in its center is co-localized with the excitation beam [149]. Thus, a wavefront shaper in terms of a helical phase ramp extending from 0 to $2 \pi$ is placed into the beam path of a circular polarized beam. This phase ramp is a polymer layer with a thickness 


\section{Experiments and results}

profile similar to that shown in Fig. 3.8, A. The beam profile of the resulting STED beam is depicted in panel B. This profile is fabricated such that the maximal thickness of the polymer corresponds to a phase retardation of $2 \pi$ for the employed STED wavelength. The occurrence of constructive and destructive interference finally creates a donut-shaped beam profile in the focus. If no adequate phase ramp is available for a certain wavelength, like for a STED wavelength of $600 \mathrm{~nm}$ used to deplete the fluorescence of ATTO532, the beam shape can alternatively be induced with a spatial light modulator (SLM).

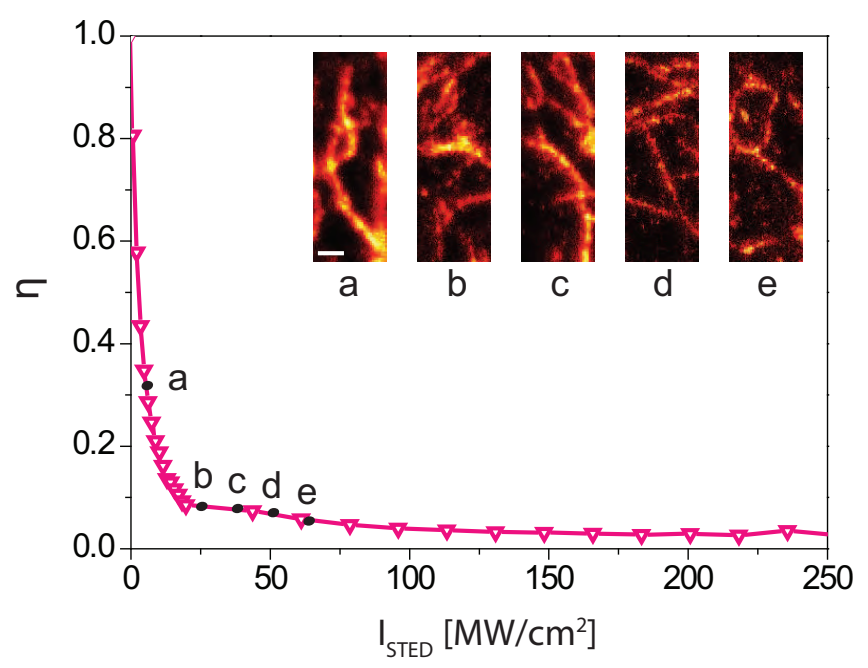

Figure 3.9: Depletion factor $\eta$ plotted against the intensity of the STED beam on sections of PtK cells in Nanoplast. Vimentin was labeled with ATTO647N, section thickness $65 \mathrm{~nm}$. The plot was measured with the diffraction limited foci of the spatially overlapped excitation and STED beams. $\eta$ is corrected for occurring reexcitation. To demonstrate the corresponding resolution enhancement with STED, pictures of the same sample after applying a donut shaped beam with an adapted intensity in the microscope aperture are shown in a-e. Scale bar: $500 \mathrm{~nm}$.

As the STED light spectrally overlaps with the fluorescence, a bandpass filter is needed in the detection path to separate the fluorescence from the laser light. For the detection of the single fluorescence photons, normally an avalanche photo diode (APD) is used. The sample is moved by a piezo scanning stage, while the objective lens (OL, 100x, NA 40 oil immersion, PL APO, Leica Microsystems) is stationary.

To characterize the fluorescence depletion due to STED in ultrathin sections, the depletion factor $\eta$ of ATTO647N was measured at this setup. To determine the depletion factor, a $3 \mu \mathrm{m} \times 3 \mu \mathrm{m}$ area of an immuno-stained sample was scanned with the pair of excitation and STED beams (without a wavefront shaper) and subsequently with the excitation beam and the STED beam only. In this experiment, the excitation power was chosen to be $4.5 \mu \mathrm{W}$ with a step size of $20 \mathrm{~nm}$ and a pixel dwelltime of $50 \mu \mathrm{s}$. For both cases the overall fluorescence intensity was measured. The depletion factor was calcu- 
lated by dividing the fluorescence intensity in presence of the STED beam by the one with excitation beam only. Afterwards, $\eta$ was corrected for reexcitation by the STED beam.

\section{Results}

The resulting plot of the depletion factor $\eta$ against the corresponding STED intensity $I_{\text {STED }}$ is demonstrated in Fig. 3.9. The STED-induced transition from the on- to the off-state can be effectively achieved at comparably low STED intensities. Moreover, the transition is saturable and at STED intensities $I_{\text {STED }} \gtrsim 100 \mathrm{MW} / \mathrm{cm}^{2}$, less than $4 \%$ of the molecules still remain in the fluorescent on-state, while the majority was switched back to the off-state.

To visualize the influence of the STED intensity on the fluorescence depletion efficiency, although foreclosing the results of the upcoming paragraph, STED images for five different values of $I_{\text {STED }}$ marked in the plot (a-e) are shown as well. For these measurements a donut shaped STED beam was employed. It is clearly obvious that the resolution of cellular fibers, represented by the arbitrary thickness of the filaments, is enhanced subsequently with higher values for $I_{\text {STED }}$. At a STED intensity of $70 \mathrm{MW} / \mathrm{cm}^{2}$ the determined mean diameter of the vimentin tubules was reduced to $45 \mathrm{~nm}$.

\subsubsection{STED imaging on ultrathin sections}

That STED imaging can be assigned to ultrathin sections which intrinsically already provide an enhanced resolution in axial direction is substantiated for three different dyes (ATTO532, ATTO590 and ATTO647N) at three different setups. The test structures were chosen because they were either well-known from former experiments ( $\beta$-tubulin) or currently highly discussed and therefore of interest (Histone, Golgi protein).

\section{Methods}

For all three test structures cell culture samples were PFA-fixed and immuno-stained. Afterwards they were embedded into Nanoplast and subsequently ultrathin sectioned with an ultramicrotome (EM UC6, Leica Microsystems GmbH, Wetzlar, Germany). The sections were looped up and transferred to plasma-cleaned glass coverslips. Finally, they were embedded in mowiol and imaged. The preparation protocols are described in detail in the Appendix. For each sample a specific setup was applied, which for each case has been described in detail elsewhere [124, 180, 58].

\section{Results and discussion}

Panel A of Fig. 3.10 shows $\beta$-tubulin stained with ATTO532. As a hetero-dimer together with $\alpha$-tubulin, $\beta$-tubulin forms the branched network of microtubles pervading a cell [133]. These microtubles are the thickest fibrous structure of the cytoskeleton $(20-28 \mathrm{~nm}$ in diameter). They serve as transport rails in many cellular processes including the chromosomal segregation [50]. Very often two or more of the fibers are contorted, such 


\section{Experiments and results}

that they appear as a single thick strand in a confocal microscope. Even with the lateral high resolution of a STED microscope, two fibers grown on top of each other are difficult to distinguish within the focal spot. Thus, only with the combination of ultrathin sectioning and high-resolution imaging, the twisted microtubles can be clearly resolved.

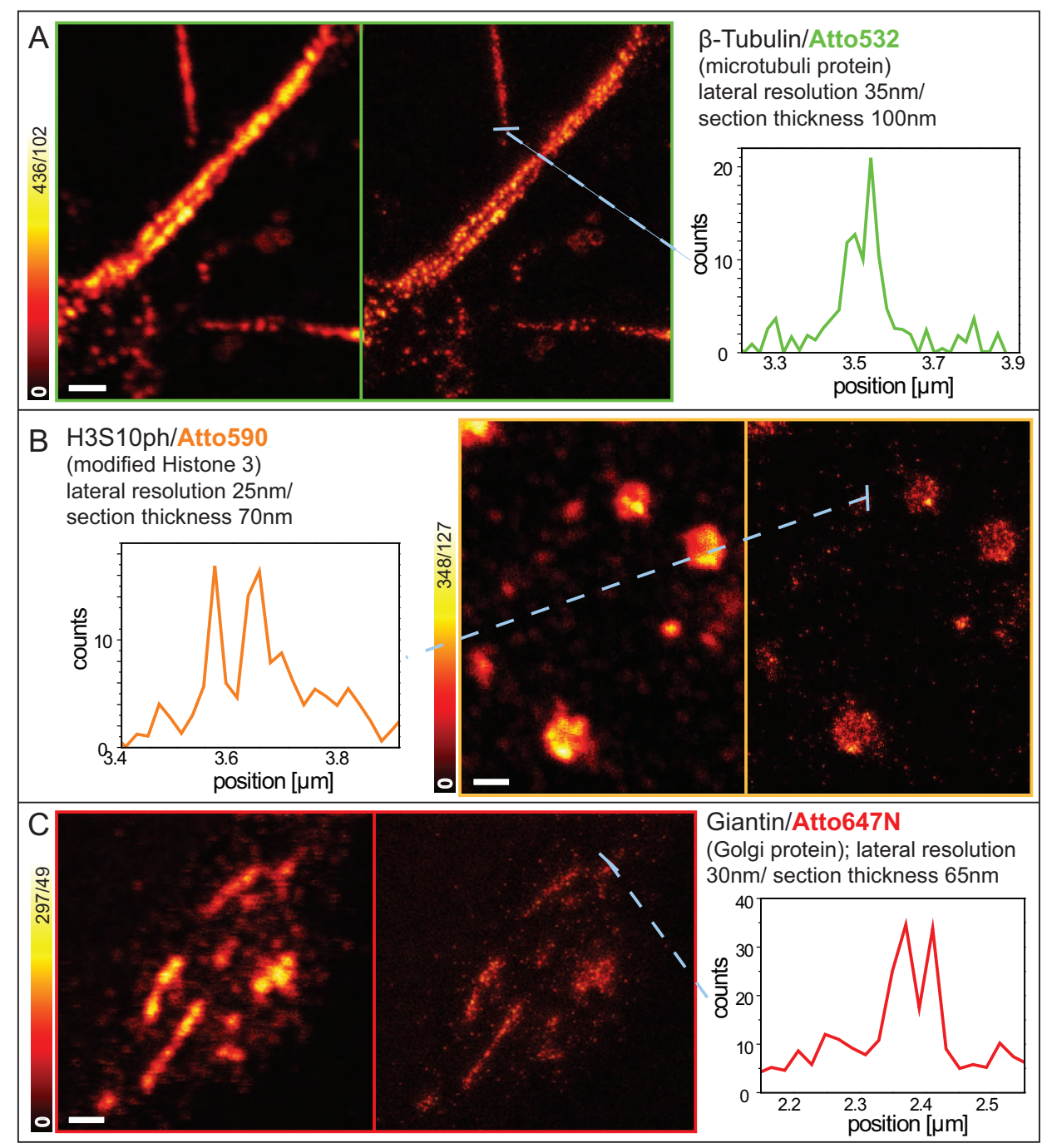

Figure 3.10: Confocal (left) and STED (right) of three different cellular structures after immuno-staining, embedding in Nanoplast and ultrathin sectioning. The lateral resolution and the section thickness are as designated. In addition a line profile is shown for each structure. (A): $\beta$-Tubulin stained with ATTO532, PtK cells; (B): Modified Histone3 stained with ATTO590, Hela cells; (C) Giantin stained with ATTO647N, BHK cells. Note that for the latter a dye-conjugated Fab fragment was used for labeling instead of a secondary antibody. Scale bars: $1 \mu \mathrm{m}$. 
In panel B of Fig. 3.10, a modified histone H3S10ph is immuno-stained with ATTO590. Histones are part of the chromatin structure in eukaryotic cell nuclei. They play a crucial role in the packing of the DNA, although so far the exact structure and the packing mechanism of chromatin are not completely understood [8]. By modifying parts of one of the five histones ( $\mathrm{H} 1, \mathrm{H} 2 \mathrm{~A}, \mathrm{H} 2 \mathrm{~B}, \mathrm{H} 3, \mathrm{H} 4)$, one is trying to assign distinct functions to a certain histone. For H3S10ph, it could be shown that this modified form of H3 is not randomly distributed over the nucleus, but is only found in certain spherical regions. This could not be revealed by confocal microscopy on the whole cell, where the whole nucleus appeared as a continuous blurry structure. The enhanced resolution in all spatial directions unraveled a certain clustering of the protein, indicating that also in the spherical regions the protein was not thoroughly modified.

Finally, in panel C of Fig. 3.10, Giantin, a protein located at the trans-membrane of the Golgi-apparatus, which is a membrane enclosed reaction space in eukaryotic cells, is depicted. It also occurs within transport vesicles in the close environment of the apparatus [114]. By addressing Giantin, fragmentary parts of the Golgi membrane could be observed, which are in all three dimensions too close for observation with a conventional microscope. To ensure an even higher quality staining, instead of a secondary antibody a Fab-fragment was employed. The latter is only one third of an antibody in size [110]. However, it involves a less effective labeling with fluorescent dyes of the marker and induces a less bright staining of the structure. Thus, it is not globally applicable, but should be especially considered if proteins within extremely densely packed structures are of interest.

\subsubsection{STED imaging of ultrathin sectioned vs. bulk structures}

After the principle of STED imaging has been shown to work on ultrathin sections, this paragraph outlines the particular advantages of ultrathin sectioning over imaging of bulk structures. A lateral STED donut is roughly extended to $600 \mathrm{~nm}$ in the axial direction. The detected image is a projection of all fluorescent features within this comparably thick layer into the image plane. In consequence, very densely labeled structures which lay on top of each other are difficult to resolve in this range. In addition, dim structures are easily obscured by adjacent bright features and cannot be detected anymore. By questioning an ultrathin section of a thinness well below $100 \mathrm{~nm}$ those problems are substantially diminished. Above all, as every single section is addressed independently, distracting background signal from fluorescent structures in the periphery of the focal layer is largely omitted. Again this enhances the imaging contrast and is essential for the detection of dim features.

What is even more pivotal in many applications is the fact that one is not restricted by the penetration depth of visible light into a scattering tissue. Therefore, arbitrary thick samples can be entirely addressed. Within that, photo-bleaching in adjacent layers to the focal volume is avoided by the independent examination of each section. Thus, during the imaging of the subsequent sections, one can take advantage of the initial, unaffected fluorescence in each section. 


\section{Experiments and results}

\section{Methods}

To compare ultrathin sectioned and bulk specimens, two equal samples of four different immuno-stained samples each were prepared. Densely labeled structures were chosen at this point. One sample of each structure was right after fixation embedded in Mowiol. The other was embedded in Nanoplast and ultrathin sectioned according to the protocol described in the Appendix. The section thickness varied from $100 \mathrm{~nm}$ down to $65 \mathrm{~nm}$. The slices were looped up on glass coverslips and embedded in Mowiol as well. Bulk and sectioned structures were imaged subsequently under identical conditions. The applied setups were previously described in [124, 26] and [180].

\section{Results and discussion}

For each of the bulk structures an exemplary resulting image is shown in the top row of Fig. 3.11. The background of the inset STED image shows the corresponding confocal image. The according data obtained from the ultrathin sectioned structures are depicted in the bottom row of Fig. 3.11. After ultrathin sectioning, a resolution refinement in the axial direction is already obvious in both, the confocal and the STED image. This holds for filamentous structures like Vimentin, as well as for spotty structures. On top, the combination with STED imaging generates an entire high resolution image in all spatial directions, which allows to precisely study the assembly and the clustering behavior of certain proteins.

Vimentin is a type 3 intermediate filament of the desmine family and part of the cytoskeleton of mesenchymal cells [42]. It forms a tight three-dimensional network. Hence, the imaging of reduced sample volumes generated by ultrathin slices provide an essential improvement of the SNR (see Fig. 3.10, C).

Sc35 is an example for the observation of spotty structures. As a nuclear speckle marker of the serine/arginine-rich (SR) proteins it plays a crucial role in the alternative splicing process [127]. It is localized in the cell nucleus, where proteins and genetic material are extremely tightly packed. So far, single protein clusters could only be precisely localized by means of electron microscopy in combination with ultrathin sectioning [130]. In the compact cellular nucleus, several distinct proteins, e.g. Sc35, are to close to be distinguished, even by high resolution methods. However, for selective studies on distinct nuclear proteins, optical high resolution methods are highly preferable.

Further, an examination of synaptic vesicles in neurons was carried out. To this end, synaptophysin, a well-established vesicle marker was employed (Fig. 3.11, D) [92. At a synapse the single vesicles are found in close proximity to each other, forming the so called vesicle pool [141. If one is interested in how single vesicles are taken up and what is the fate of vesicles that have recently been incorporated at the synapse, a high resolution in the lateral and axial direction is obligatory.

With respect to neurons, this is not only the case for synaptic vesicle proteins. Also, proteins that are found in the neuronal membrane can much more precisely be observed after ultrathin sectioning. Hence, SNAP-25, a part of the exocytotic fusion complex of synaptic vesicles, is depicted in Fig. 3.11, B [9]. Without sectioning one would for 
instance not be able to distinguish SNAP-25 clusters localized on the cellular membrane from those on the vesicle surface.

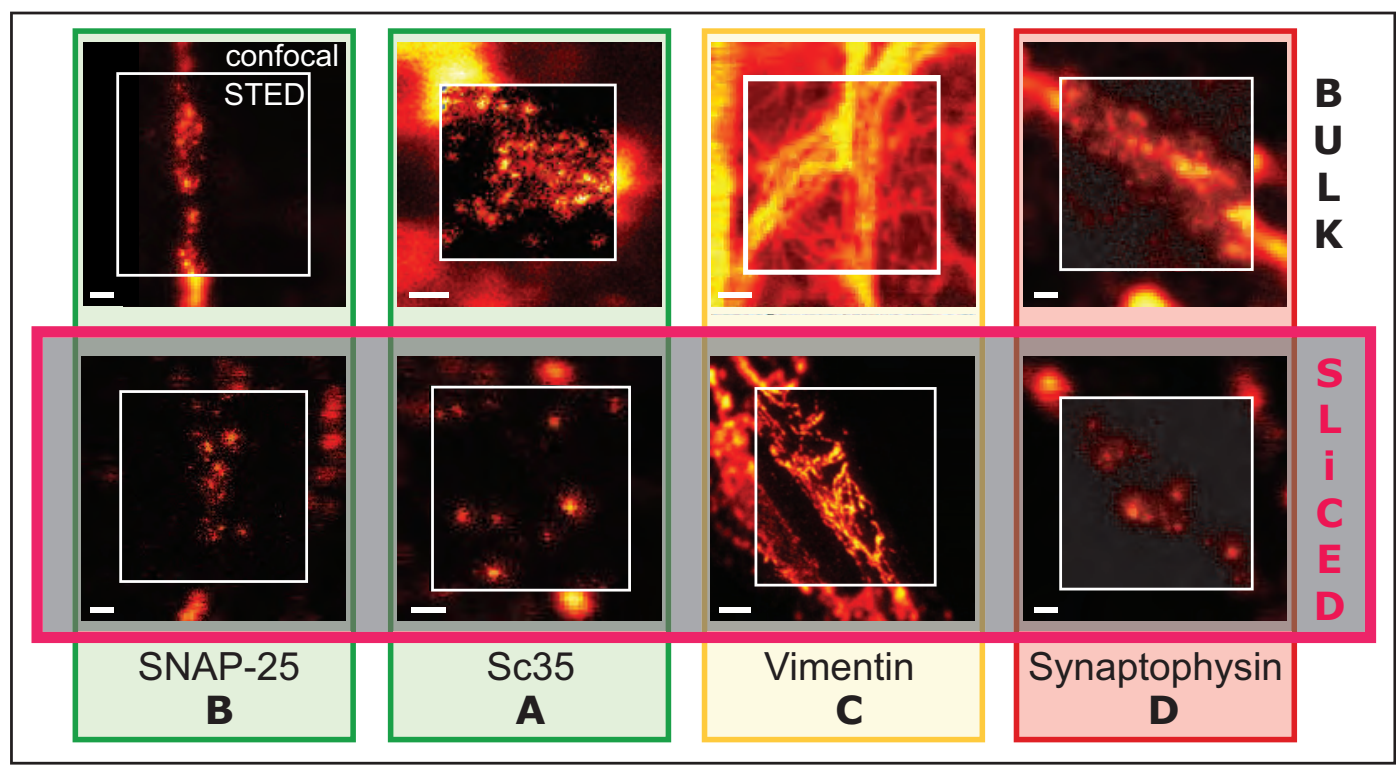

Figure 3.11: Representative confocal (shown in the background) and STED (in the insets) images of four different immuno-stained structures after embedding into Nanoplast. Top row: Images of the bulk structure in mowiol, bottom row: Images of the same structure on ultrathin sections. The corresponding excitation wavelength is indicated by the colored box for each structure. (A): Sc35 in Hela cells labeled with ATTO532. Scale bar $300 \mathrm{~nm}$; (B): SNAP-25 in neurons labeled with ATTO532. Scale bar $200 \mathrm{~nm}$; (C): Vimentin in neurons labeled with ATTO590. Scale bar $1 \mu \mathrm{m}$; (D) Synaptophysin in neurons labeled with ATTO633, scalebar $200 \mathrm{~nm}$.

Within these experiments, only mono-layer cultured cells were employed. One can estimate the benefit, which can be expected for even more spatially extended samples like whole organs or animals. Not only that focusing deep into these samples is severely limited by aberration and scattering effects, but also the background signal from peripheral layers would be highly distracting.

\subsubsection{Ultrathin mechanical vs. optical sectioning (isoSTED) in STED nanoscopy}

As was shown in the last Section, subdiffractional resolution in all three spatial dimensions strongly improves the light microscopic imaging of numerous biological structures. In the following this is verified on mitochondria and in particular on their inner- and outer membrane. To precisely localize different proteins within either membrane, an entire high resolution in three dimensions is mandatory. 


\section{Experiments and results}

Mitochondria are the power machines of eukaryotic cells [145]. In most cell types, they consist as a dynamic reticulum of long, thin and branched tubuli spreading throughout the cytoplasm of the cell. As an example, a PtK cell immuno-stained for the TOM complex, a protein of the outer mitochondrial membrane, is depicted in Fig. 3.13, A. Mitochondria are shown in green, while the DAPI staining of the DNA in the cell nucleus is colored in blue. Additionally, an immuno-staining of $\beta$-tubulin is shown in red. Typically, mitochondria are about $200-400 \mathrm{~nm}$ in diameter, which corresponds to about a third of the wavelength of visible light (see Fig. 3.13 A, inset). Although light microscopy is the method of choice for studying mitochondrial shape and their dynamic behavior, details about their architecture could so far only be obtained from electron microscopy [10, 94]. The existence of an inner and an outer membrane for example has been known for about 50 years now [39. Light microscopy concepts have been ill-suited to this end because of their poor lateral and axial resolution. However, various recent developments provide a nanoscopic resolution in all three dimensions and were applied also for the imaging of mitochondria [11, 86, 26].

Still, the inner membrane of the organelles, which forms the christae, is strongly threedimensional. Thus, it is hardly accessible and still remained a challenging task for light nanoscopists. At the same time it is highly rewarding since many cellular processes in mitochondria take place at or within the inner mitochondrial membrane. Hence, in this Section the capacity of the mechanical ultrathin sectioning approach in combination with STED imaging is verified. In addition, a comparison with the results obtained by optical sectioning in terms of isoSTED imaging is presented.

\section{Methods}

The detailed staining protocol is described in the Appendix. To prepare the samples, the inner mitochondrial membrane was marked by attaching fluorophore-labeled antibodies to one of the major protein complexes of the oxidative phosphorylation system within the inner membrane, the $\mathrm{F}_{1} \mathrm{~F}_{0}$ ATPase. For mechanical sectioning the fixed cells were embedded in Nanoplast, ultrathin sectioned $(50 \mathrm{~nm})$ and finally embedded in TDE. TDE is advantageous since it matches the refractive index of the immersion oil and remarkably enhances the observed fluorescence signal.

For the imaging of ultrathin sections a STED setup with a supercontinuum laser source (SC-450 HP, Fianium, Southampton, UK) was used [180]. In principle, the setup is similar to that shown in Fig. 3.7. As both the excitation and STED pulses are generated by the same laser source, the setup is simpler, user-friendly and less expensive. The excitation wavelength was chosen to be $\lambda_{e x c}=570 \mathrm{~nm}$ and extracted by a bandpass filter, whereas the STED beam with a wavelength $\lambda_{S T E D}=700 \mathrm{~nm}$ was extracted by a wavelength selector built around a highly dispersive Brewster prism (SF59, Schott AG, Mainz, Germany). To enhance the power of the STED beam, which allows for a higher lateral resolution, two orthogonally polarized but otherwise equal intensity beams of the STED wavelength were extracted from the spectrum. These beams were individually guided through two separated beampaths, including two vortex phase masks (RPC Photonics, Rochester, NY, USA) providing the toroidal beam shape, and overlaid before guiding 
them into the objective lens (PL APO $100 \mathrm{x} / 1.40$ - 0.7 oil, Leica Microsystems, Wetzlar, Germany). This yields a time-averaged power of a few $\mathrm{mW}$ at the aperture of the objective lens, corresponding to a pulse energy of about $7 \mathrm{~nJ}$. The extracted laser pulses were already as along as about $80 \mathrm{ps}$ at a repetition rate of $1 \mathrm{MHz}$ and thus additional pulse stretching was unnecessary. The detection was performed by an avalanche photodiode module (SPCM-AQRH-13-FC, Perkin-Elmer, Quebec, Canada), combined with a time-correlated single-photon counting board (SPC-830, Becker \& Hickl GmbH, Berlin, Germany). Typically, the STED and confocal reference images were recorded quasi simultaneously on a line-by-line basis by opening and closing a shutter in the STED beam. In these experiments a pixel size of $10 \mathrm{~nm} \times 10 \mathrm{~nm}$ together with a pixel dwelltime of $20 \mu \mathrm{s}$ was used. For scanning the samples a piezo stage (NanoBlock, Melles Griot GmbH, Bensheim, Germany) was used. The resolution on biological samples could be proven to be in the range of $20 \mathrm{~nm}$.

The isoSTED imaging was performed with the setup described elsewhere1 [147]. To this end, the immuno-stained samples were fixed with $8 \%$ PFA and afterwards embedded in TDE by applying a subsequent dilution series [161.

\section{Results and discussion}

Mechanical ultrathin slicing and subsequent STED imaging clearly enabled the delineation of the inner-membrane flow, disclosing christae as pronounced substructures inside these organelles. The diameter of the mitochondrial christae estimated from the fluorescence signal was found to be around $30 \mathrm{~nm}$, which is in agreement with the data obtained by electron microscopy where the pure unlabeled membrane is visualized [39] (Fig. 3.12).

The figure shows an electron-microscopic image of the mitochondrial membranes (Panel A) compared to a STED image of the inner membrane (Panel B). Similar regions in both images are depicted as magnified insets. Although STED microscopy can still not compete with electron microscopy in terms of resolution, a similar thickness and arrangement of the inner membrane is observed by both techniques. This is also indicated by the peak maxima distance in the lineprofile shown. Due to the required (immuno- or other) labeling in light microscopic techniques, the inner membrane is slightly broadened. Hence, new and smaller affinity binders, like Fab fragments or Fluorescein arsenical helix binders (FlAsH), are desirable.

However, mitochondria are of considerable interest, since numerous severe human diseases are caused by defects in the assembly of the oxidative phosphorylation system in the christae [158]. For further effective investigations in this field, three dimensional high resolution imaging, which allows the use of selective protein markers is mandatory. In this respect, light-microscopic methods are definitely favorable over electron-microscopic techniques. Apart from the approach explored in this work, which combines optical high resolution STED imaging with mechanical ultrathin sectioning, Schmidt et al recently published an all-over optical sectioning technique to generate nanoscopic resolution in all spatial directions [147].

\footnotetext{
${ }^{1}$ All isoSTED measurements were performed by Roman Schmidt.
} 


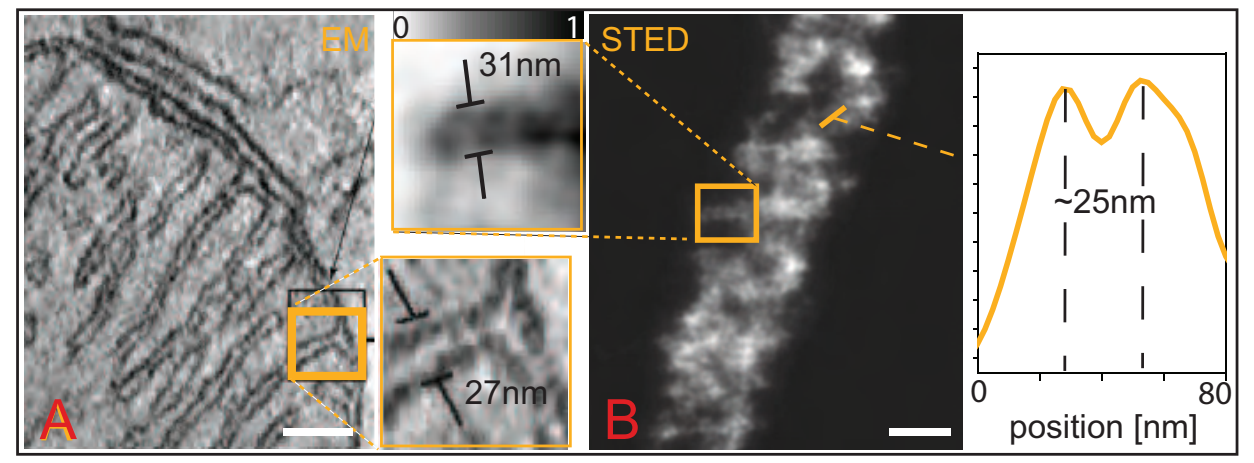

Figure 3.12: Lineup of an exemplary EM image 39] of an ultrathin sectioned chick cerebellum cell and a STED image of an immuno-stained and ultrathin sectioned PtK2 cell (B). The colormap in the enlarged box was inverted by means of the large image to underscore the similarity to the EM data. With both techniques the diameter of mitochondrial christae was observed to be about $30 \mathrm{~nm}$. Note that, beside the intrinsically worse resolution, structures in the STED image are slightly broader because of the attached antibody/dyelabel, which was not necessary in EM. Also note that the outer mitochondrial membrane is visible in the EM, but was not stained for the STED image. A lineprofile of the STED image is additionally shown. Scale bars: $50 \mathrm{~nm}$.

isoSTED nanoscopy provides sub-diffractional resolution even in whole, intact cells by scanning a nanoscopic nearly spherical effective PSF through the sample (see sketch of effective excitation patterns in Fig. 3.13, D, bottom). The latter is generated by overlapping the excitation spot of two opposing high NA oil objectives with a hollow sphere of light for switching off the fluorescence by STED. Effectively the spot is about $30 \mathrm{~nm}$ in diameter and consequently about three orders of magnitude smaller than that of a confocal microscope (Fig. 3.13, D, top). Essentially operating the system as a beam scanning microscope, any single layer can be addressed within a semi-thick sample (about $200 \mu \mathrm{m}$ ) this way [70]. The revolutionary potential of this non-invasive approach has been shown in various applications [170, 71]. However, the complex setup requires a specialist as operator.

Exemplary images derived with both methods are shown in Fig. 3.13, together with a small sketch commenting on the workflow. Panel B shows STED images of ultrathin sections. Panel C shows isoSTED images recorded on whole intact cells [148].

In both cases a quite heterogeneous arrangement of the christae was observed even within a single mitochondrial tubule. Regions of stacked christae lamella are altering with regions of up to $\sim 10^{5} \mathrm{~nm}^{2}$, which were devoid of christae. In most cases the christae were perpendicular to the direction of the mitochondrial tubule, but christae running along its length axis can also be found, underscoring that their arrangement is highly variable. Most explicitly the membrane's course is visible when the tubule crosses the imaged plane and a perpendicular crossection is detected.

Comparing both imaging methods, mechanical sectioning typically does not reach a 
section thickness as thin as $30 \mathrm{~nm}$ but yields slices of $50 \mathrm{~nm}$ only in reproducible quality. Still, this does not mean that the axial resolution is effectively poorer. Lacking any offfocal-plane fluorescence emission, the mechanical slicing approach tends to deliver images with a throughout higher contrast compared to all-optical sectioning.

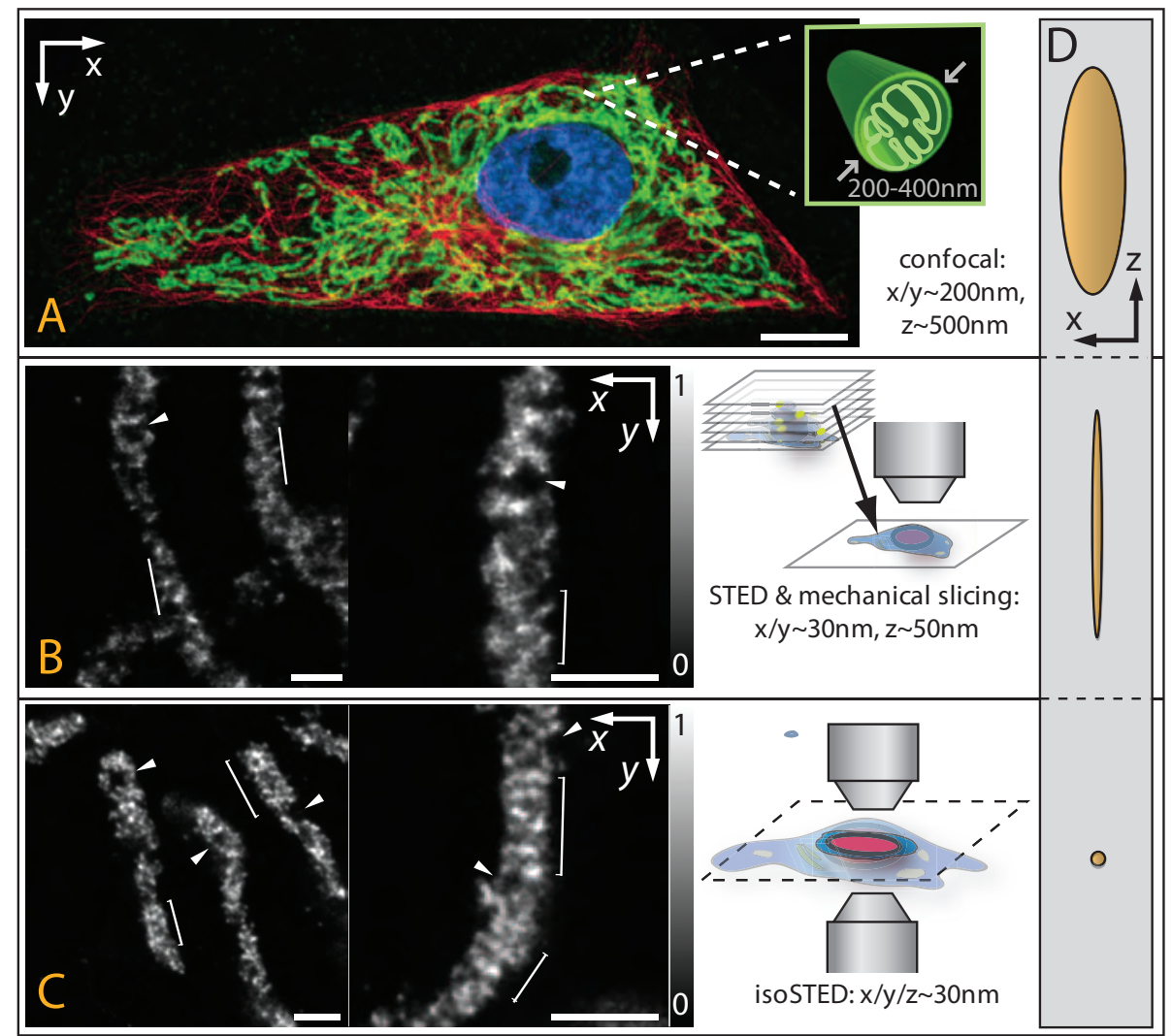

Figure 3.13: A: Confocal overview over the mitochondrial network of a fluorescent labeled PtK cell: Mitochondria visualized by antibody-labeling of the TOM complex on the outer mitochondrial membrane (green); microtuble cytoskeleton immuno-stained against $\beta$-tubulin (red); DAPI- labeling of the nucleus (blue). The inset shows a schematic crossection of a single mitochondrium. B: Overview and detailed STED images showing the arrangement of christae in intact wild type PtK cells. The latter were labeled by antibodies against $\mathrm{F}_{1} \mathrm{~F}_{0}$ ATPase. After embedding in Nanoplast, ultrathin slicing $(50 \mathrm{~nm})$ were generated mechanically. C: isoSTED images of the same structures as in B in a whole cell, embedded in TDE. B/C right: Sketch describing the imaging process. STED images were deconvolved using a maximum-likelihood expectation maximization algorithm. D: Effective PSFs of the different imaging modes. The size of the PSFs is scaled to the inset in A showing the schematic crossection of a mitochondrium. Scale bars $500 \mathrm{~nm}$. 
What is even more crucial is the fact that with regard to optical sectioning, both the excitation and the STED laser beams photo-bleach a large amount of fluorophores in the surrounding of the focal plane, making a nondestructive imaging of several subsequent planes hardly possible with currently available fluorophores. For mechanical sectioning, this problem can be neglected, since each section is imaged completely independently.

In summary, beyond doubt it is shown that both techniques reveal matchable convincing results. However, isoSTED nanoscopy might, among other methods, at some point pave the way for three-dimensional high-resolution imaging of living cells. But due to the mentioned advantages, mechanical ultrathin sectioning provides a considerable alternative in many applications. It will of course never be capable of live-cell imaging or observing fast dynamic processes in real time. Still, with modern rapid fixation methods, certain processes on a comparably long timescale might also be followed by time-lapse imaging. 


\subsection{Application I: 3D Investigation of a cultured neuronal network}

Cultured neurons play an important role as test systems for the investigation of neuronal communication. The central part of the latter is the synaptic vesicle cycle, including the fundamental step in neuronal communication, which is the fusion of synaptic vesicles with the plasma membrane 93 . Where synapses are formed, how synaptic vesicles are filled with neurotransmitters, in which arrangement the vesicles are stored before exocytosis, what happens after their fusion into the synaptic membrane are, among further questions, of high interest for the neuroscientific community [165]. An overview of the current status of knowledge focusing on the synaptic vesicle cycle is given in Fig. 3.14. A. In particular concerning the fate of recently endocytosed synaptic vesicles, several theories are currently under debate and are, accounting this also, outlined in the sketch.

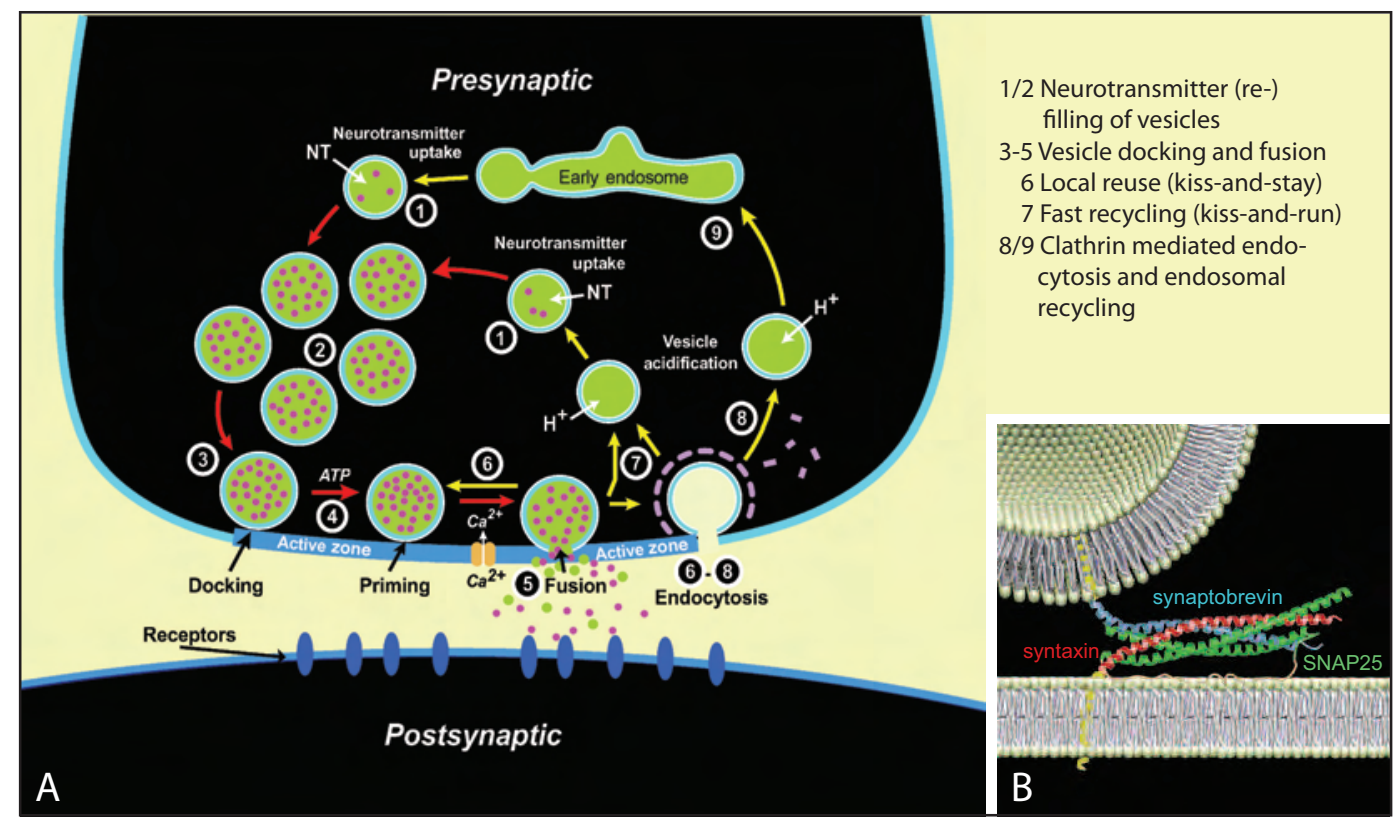

Figure 3.14: A: The synaptic vesicle cycle, adapted from [167]. Synaptic vesicles are filled with neurotransmitters by active transport (1) and from the vesicle cluster that may represent the reserve pool (2). Filled vesicles dock at the active zone (3) where they undergo a priming reaction (4) which makes them competent for $\mathrm{Ca}^{2+}$ triggered fusion-pore opening (5). Afterwards they undergo endocytosis and recycle via several routes: local reuse (6), fast recycling without an endosomal intermediate (7) or Cathrin-mediated endocytosis (8) with recycling via endosomes (9) [165]. B: Synaptic fusion complex formed out of three different SNARE proteins: synaptobrevin is located on the vesicle surface, syntaxin1 and SNAP-25 are part of the plasma membrane. 


\section{Experiments and results}

For the investigations of synaptic processes, one relies on the specific observation of single proteins with high spatial resolution in all three dimensions. That the latter can be provided by the explored approach of ultrathin sectioning in combination with STED nanoscopy, has in principle been shown in the previous Section. However, the exploration of a neuronal network poses further challenges, most prominent in terms of the precise localization of single protein clusters. This includes either the spatial relation of a certain protein to a second one or its allocation to a distinct layer of a neuronal cell, rather a synapse.

The experiments described in the individual parts of this Section provide certain approaches to solve these difficulties ${ }^{2}$

\section{Part A - Two-color imaging of a cultured neuronal network}

For the investigation of single proteins in either the neuronal membrane or in synaptic vesicles, the information one can obtain from a single color image is severely limited. Instead, it is very often requested to explore two different structures and their assembly relative to each other with high resolution methods. Therefore, two-color STED imaging on ultrathin sections was carried out and is described in the following.

\section{Methods}

Cultured rat hippocampal neurons were fixed with PFA and subsequently immunolabeled for two proteins: synataxin 1 and $\beta$-tubulin. Secondary antibodies labeled with ATTO532 or ATTO633 were used, followed by another fixation step with PFA. The spectra of the applied fluorescent dyes, together with the spectral position of the excitation, the detection and the STED light are depicted in Fig. 3.15. A. To enable an accurate registration of the two different color channels, the immuno-stained samples were incubated with a diluted suspension of two-color fluorescent silica beads at $37^{\circ} \mathrm{C}$. A detailed staining protocol is given in the Appendix.

The beads were purchased as green-fluorescent beads with an $\mathrm{NH}_{2}$-functionalized surface (sicastar greenF, $300 \mathrm{~nm}, 50 \mathrm{mg} / \mathrm{ml}$, micromod GmbH, Rostock, Germany) and were afterwards dyed with ATTO633-NHS-ester yielding a fiducial marker visible in both detection channels. Silica beads were chosen because of their hardness, going along with nice cutting qualities, and because the dye had been chemically incorporated into the substrates, so that a bleeding out of the staining during the embedding procedure and in particular in an unpolar environment is prevented.

After bead-incubation the samples were embedded in Nanoplast resin [5]. To this end, the monomer solution was added onto the coverslips using BEEM capsules as a mold. The polymerized blocks were detached from the coverslips by dipping them into liquid nitrogen, trimmed and cut with an ultramicrotome (EM UC6, Leica Microsystems GmbH, Wetzlar, Germany) and an ultrasonic diamond knife (Diatome AG, Biel, Switzerland) to a thickness of $100 \mathrm{~nm}$. To avoid back reflections of the laser beam during imaging at the

\footnotetext{
${ }^{2}$ The presented experiments were performed in collaboration with Silvio Rizzoli (European Neuroscience Institute, Göttingen, Germany), who prepared the immuno-stained samples.
} 


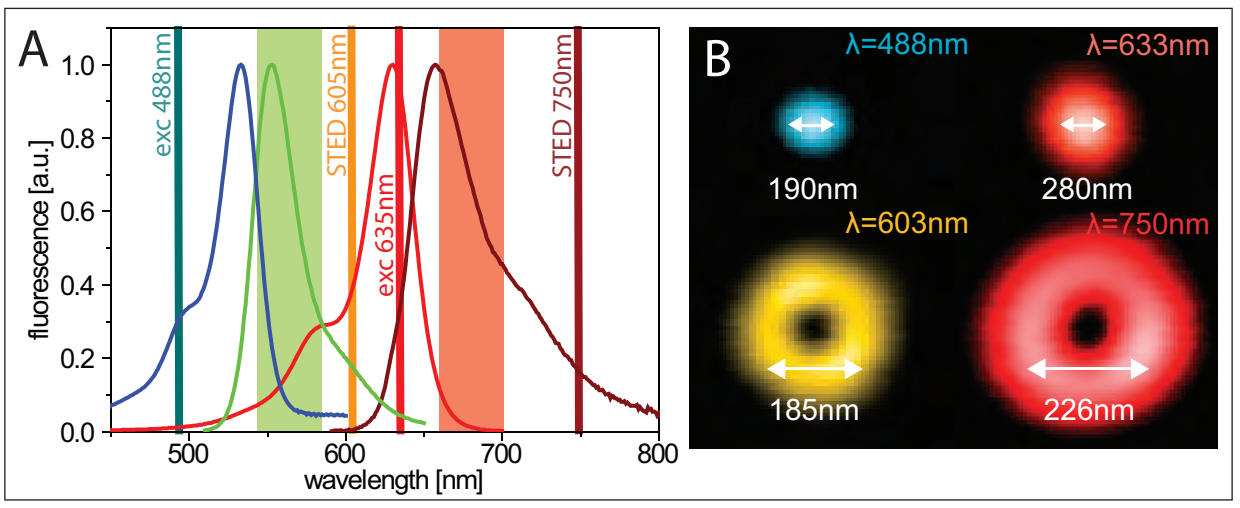

Figure 3.15: A: Absorption and fluorescence spectra of the two applied dyes. The excitation and STED beams, as well as the detection range are also indicated. B: Focal intensity distribution of each beam and it's FWHM.

interface between the section and air, the sections were capped with another coverslip that was previously spin-coated with a thin layer of Mowiol.

For STED data acquisition a home-built two-color STED microscope [124, 24, 26], capable of imaging a visible and an infrared dye simultaneously with high lateral resolution $(\approx 30 \mathrm{~nm})$, was employed. Briefly, a laser system consisting of a Ti:Sapphire master oscillator (Mira 900F, Coherent, Santa Clara, CA), a regenerative amplifier (RegA 9000, Coherent) and an optical-parametric amplifier (OPA 9400, Coherent) was used to generate STED pulses at a rate of $250 \mathrm{kHz}$ and a wavelength of $603 \mathrm{~nm}$ for the visible STED beam. For the infrared STED beam a second mode-locked Ti:Sapphire oscillator (Mira $900 \mathrm{~F}$, Coherent) with a repetition rate of $76 \mathrm{MHz}$ was used at a wavelength of $750 \mathrm{~nm}$. The $<250 \mathrm{fs}$ duration of the initial pulses was expanded to $\approx 200 \mathrm{ps}$ by means of a glass fiber [25]. The pulse energies at the back aperture of the objective lens were up to $0.5 \mathrm{~nJ}$ and $1.5 \mathrm{~nJ}$ for the visible and infrared channels, respectively. Fluorescence excitation was performed with two picosecond diode lasers (PicoTA-488 and LDH-P-635, PicoQuant GmbH, Berlin, Germany) synchronized to the Ti:Sa oscillators. To create the toroidal STED donut, a vortex phase plate (RPC Photonics, Rochester, NY) for the infrared channel and a spatial light modulator (Hamamatsu, Hamamatsu City, Japan) for the visible channel were placed into the expanded laser beams before focusing them with a 100x/NA 1.4 oil immersion lens (Leica Microsystems GmbH, Wetzlar, Germany). The generated excitation and STED PSFs for both color channels are depicted in Fig. 3.15 , B.

The images of the infrared and visible channels were recorded consecutively, whereas the STED and the confocal images were measured quasi-simultaneously on a line-byline basis. The field of view was typically chosen as $10 \mu \mathrm{m} x 10 \mu \mathrm{m}$, which along with a pixel size of $20 \mathrm{~nm}$ and a pixel dwell time of $0.5 \mathrm{~ms}$ for the infrared and $8 \mathrm{~ms}$ for the visible channel, resulted in acquisition times of $\sim 60 \mathrm{~min}$ per two-color image. The image acquisition was performed with the custom written software Imspector [150]. 


\section{Experiments and results}

As ATTO532 has a markedly tendency to form a triplet state upon excitation, a laser pulse frequency of $250 \mathrm{kHz}$ was applied to guarantee a relaxation of the excited dye molecules into the singlet ground state before the next pulse arrives [25]. Thus the acquisition time was extraordinarily long. This entailed a drift of the piezo stage appearing as a shearing of the image, which did not occur in the fast infrared channel. Hence a simple superposition of the two color channels was no more practicable.

By means of the two-color beads this drift could be corrected. To this end, the beads were manually selected in the individual images of each color-channel. With customwritten routines in MATLAB (The Mathworks, Natick, CA, USA), a polynomial drift was fitted to the selected bead positions.
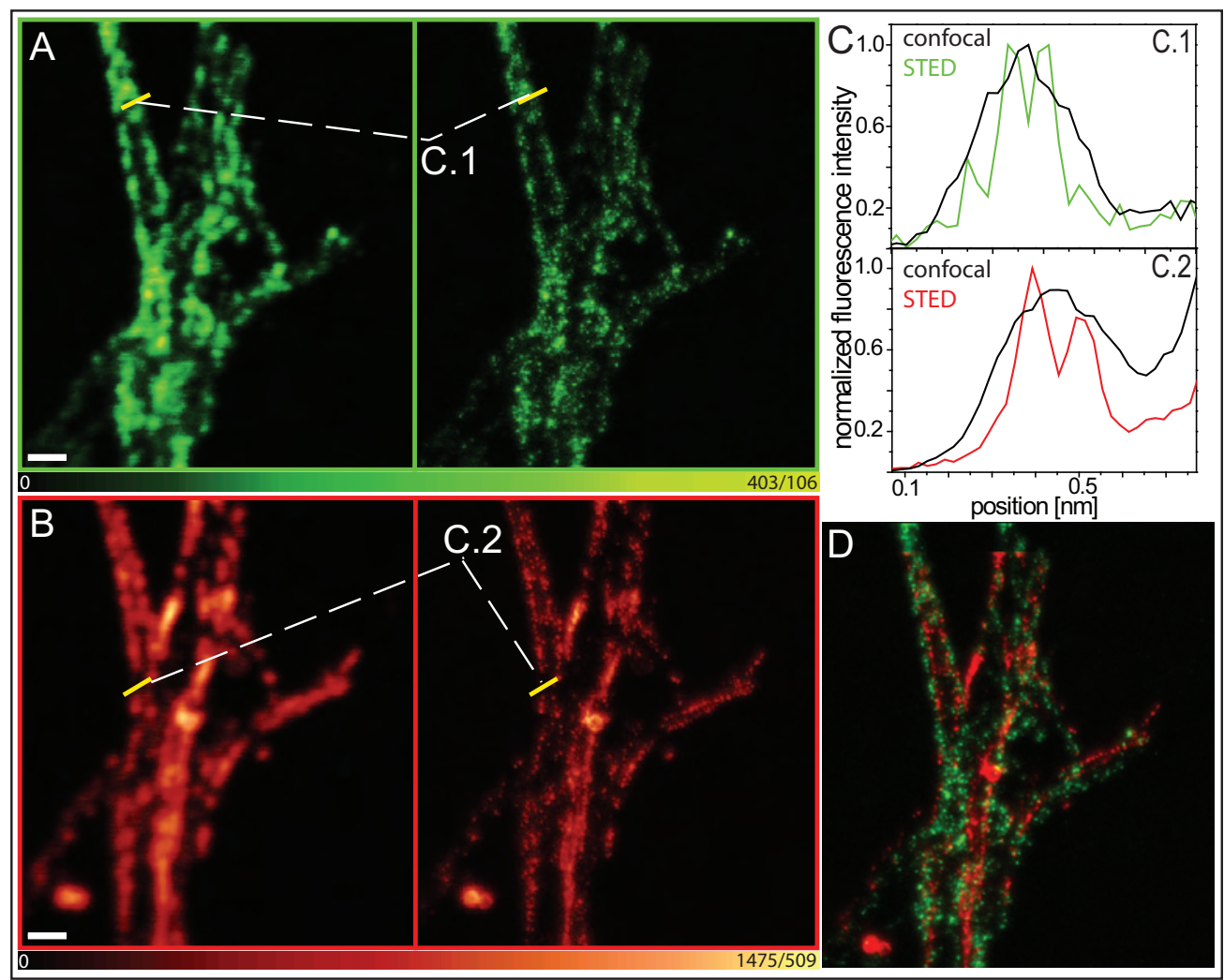

Figure 3.16: Confocal (left) and STED (right) images of an immuno-stained, ultrathin sections of a cultured neuronal network. Syntaxin1 is labeled with ATTO532 (A), $\beta$-tubulin with ATTO633 (B). The section thickness is $100 \mathrm{~nm}$, the scale bar is $1 \mu \mathrm{m}$. Panel $\mathrm{C}$ shows intensity profiles of the confocal and the STED images along the yellow lines in A (C.1) and B (C.2). STED imaging clearly allows one to resolve single features with a proximity below the diffraction limit. In D an overlay of the two STED images is shown. 


\section{Results and discussion}

A typical two-color image of a sample prepared as described above is shown in Fig. 3.16, D. The individual confocal and STED images are depicted in Fig.3.16, A (syntaxin1 immuno-stained with ATTO532, green) and B ( $\beta$-tubulin immuno-stained with ATTO633, red) respectively. Intensity profiles for both confocal and STED images along the indicated lines are plotted in Fig. 3.16, C for both colors. Clearly, features which had been obscured in the confocal image can be easily separated in the STED image.

On the basis of these results, various proteins occurring at a neuronal synapse can be questioned and precisely localized with respect to each other. Such colocalization studies would not have been possible by the information obtained from a single color. However, the high imaging contrast in the mechanically prepared ultrathin sections has an essential role at this point. First, the accuracy in the axial direction is substantially enhanced and second, also dim structures are more clearly visible in the resulting images.

\section{Part B - Reconstruction of a three-dimensional neuronal network}

In many applications the analysis of a single ultrathin layer of a sample is not sufficient to draw significant conclusions. Instead, it is important to embed the observations into a larger three-dimensional context. This holds in particular for the investigaton of neuronal communication, taken to the extreme in the connectome project [74, 112]. For electronmicroscopic data the process is fairly easy since one can avail of several continuous structures, e.g. membranes, which are visible in the images of subsequent sections. Consequently, considerable effort has been put into the automation of the registration process.

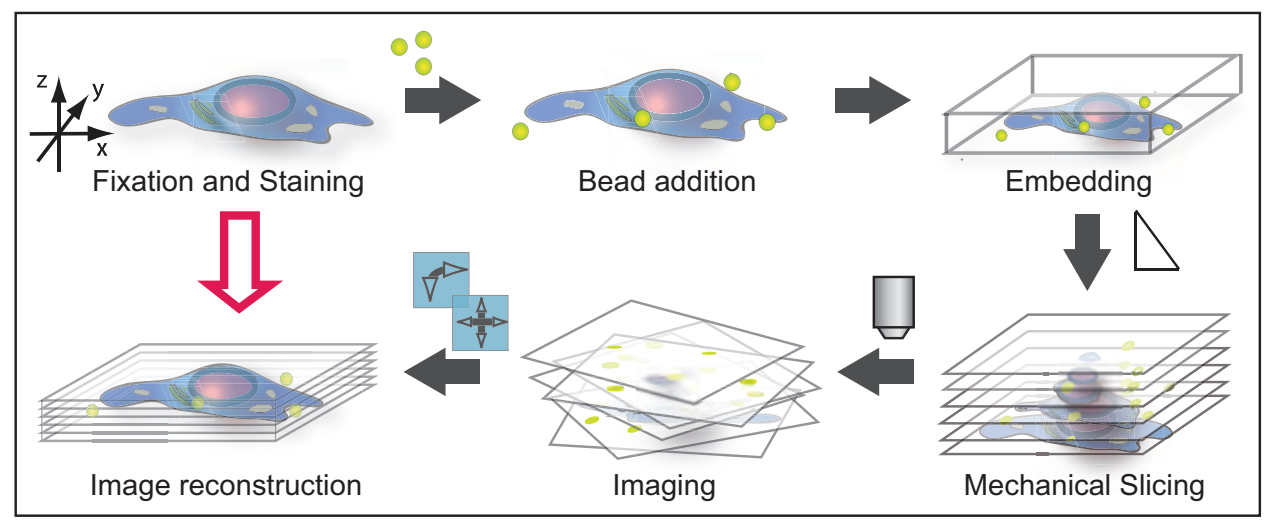

Figure 3.17: Schematic representation of the work flow for nanoscale image reconstruction in 3D: Fixed and immuno-fluorescent-stained cells are incubated with fluorescent beads, fixed and embedded in a polymer resin, followed by ultrathin slicing with an ultramicrotome. After confocal and STED imaging of the individual sections, image registration by rotation and translation allows a three dimensional reconstruction of the sample. 


\section{Experiments and results}

In contrast, in fluorescence imaging, only explicitly labeled structures are visible. If these are not continuous, but rather spotty, they are not qualified as markers for the rendering of the image stack. This problem can be evaded by a quite simple approach, namely the addition of fluorescent beads before embedding the sample into a plastic block, as outlined in the following Section.

\section{Methods}

For the three-dimensional investigation of neuronal networks, the work flow is illustrated in Fig.3.17. The sample preparation was performed in analogy to Part A. For the twocolor labeling, the same dyes were applied but directed to different proteins. This time synataxin1 had been stained with ATTO532 (green) and synaptophysin with ATTO633 (red).

The diameter of the fluorescent silica beads was chosen to be $300 \mathrm{~nm}$ in order to on one hand allow a detection of a single cut bead in three to four consecutive sections and, on the other hand, to avoid a destruction of the structure by adding too large particles onto the samples.

After ultrathin slicing, the subsequent sections were transferred onto glass coverslips, making sure to keep them in the right order. Every section was placed on an individual coverslip.

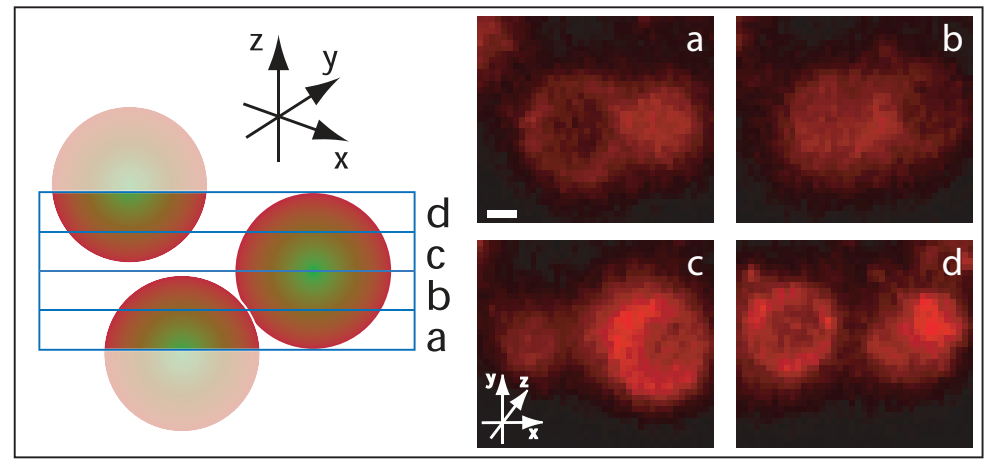

Figure 3.18: The image registration was performed with co-embedded two-color fluorescent silica-beads as anchor points. A schematic sketch of a representative bead sequence is depicted on the left side. The corresponding STED images for the red channel of the subsequent layers a-d are shown on the right. Scale bar: $100 \mathrm{~nm}$. Section thickness: $75 \mathrm{~nm}$.

The field of view in a STED microscope is much smaller than the size of a section. Hence, to generate a three-dimensional stack of images, the small region which is supposed to be imaged by STED microscopy has to be located in every subsequent section. To this end, confocal overview images of the two stained color channels were taken in advance. An example of such an image is shown in Fig.3.19, A. The region imaged with STED is also indicated by the white square. This way, the orientation on each section 
was revealed, even with the small field of view of the STED microscope. Anyway, a certain error could not be completely eliminated.

The STED imaging was performed as described above. Finally, the recorded images were superpositioned. The rotation and translation processes were performed manually with the alignment tool Midas, which is part of the academic IMOD software suite [107]. Static and animated 3D visualizations of data sets were subsequently produced with the commercial image visualization package AMIRA (Visage Imaging, Carlsbad, CA).

\section{Results and discussion}

Two-color imaging was successfully performed on fifteen $75 \mathrm{~nm}$ thin serial sections. A stack of the recorded images, each of them $10 \mu \mathrm{m} \times 10 \mu \mathrm{m}$ in size, was rendered afterwards. The slices were arbitrary oriented on the coverslips during the imaging procedure. Therefore, not only the two color channels of a single section (as described in Part A of this Section), but also the data from different sections had to be registered. For both, the co-embedded and cut fluorescent beads were used as reference marks. The appropriate protocol is briefly outlined at this point:

First, the images of a slice recorded in the red channel were superposed, taking advantage of the sectioned fluorescent beads. This procedure was performed manually. In Fig. 3.18 the principle is shown on a schematic bead ensemble which was drawn on the basis of a tru situation in the experiment. The four STED images, which can be assigned to the single layers of the sketch are also depicted. It is clearly obvious that a single bead can be found in several subsequent layers. Thus, superpositioning the centers of the bead fragments enables the image registration.

Second, the images of the green channel were correlated with the according images of the red channel for each section, which were already aligned. This was reasonable, since the images of the green channel featured an erroneous shearing, that was caused by the slow imaging process. The overlay of the two-color channels was performed computationally as described in Part A of this Section. Finally, this resulted in a rendered stack of two-color images.

A representative two-color STED image taken out of the stack is shown in Fig. 3.19, $\mathrm{B}$. The yellow arrows indicate some beads which were employed for the image registration. Panel C and D of Fig. 3.19 show a clip-out for each color channel of the image in Fig. 3.19 , B. The confocal images are also placed to visualize and underline the resolution enhancement within the focal plane which was achieved by means of STED imaging.

A stereo view of the resulting stack is depicted in Fig.3.19, E. The axial extension of the stack was $1.05 \mu \mathrm{m}$ determined by the thickness of the fifteen slices. Finally, due to the randomly orientation of the sections on the coverslips, only a small part of the imaged axon $(3 \mu \mathrm{m} \times 3 \mu \mathrm{m})$ coincided in all images. It is indicated by the white box in the two-color image in Fig. 3.19, B.

Hence, if desired, the co-localization of two proteins can be performed with a nanoscopic precision in all three dimensions of the image stack. However, for the investigation of ever larger data sets, it would be beneficial to automize the registration procedure. The same applies for the subsequent analysis of the data. 


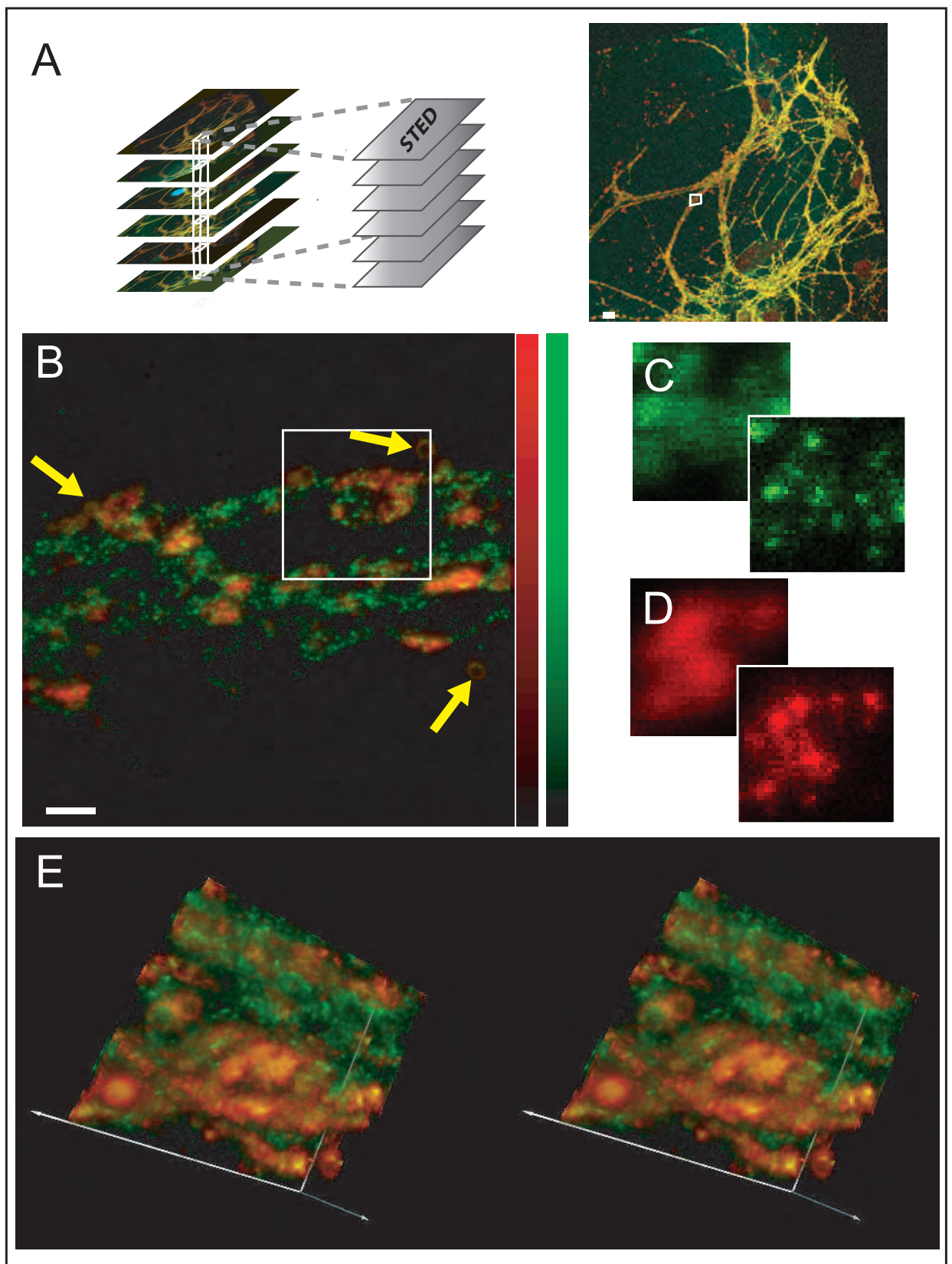

Figure 3.19: Imaging and 3D reconstruction of a cultured neuronal network (green: synataxin1, red: synaptophysin. Section thickness: $75 \mathrm{~nm}$ ). (A) Confocal overview of a single section of the series. The white square indicates the region imaged with STED. Scale bar: $10 \mu \mathrm{m}$. (B) Typical two color STED image of one slice taken out of the 3D stack. The white square indicates the region shown in $(\mathrm{E})$, scale bar $1 \mu \mathrm{m}$. Some of the fluorescent beads used for the section alignment are indicated by yellow arrows. (C) and (D): Confocal (rear) and STED (front) images of a selected region in $(\mathrm{C})$ to demonstrate the enhanced resolution in xy-direction. Size: $800 \mathrm{~nm}$ x $800 \mathrm{~nm}$. (E) Stereo image of a stack reconstructed from fourteen slices, $3 \mu \mathrm{m} \times 3 \mu \mathrm{m} \times 1.05 \mu \mathrm{m}$ 
To summarize, even the fluorescence images of neuronal proteins, which form separated clusters, a three-dimensional reconstruction of high-resolution fluorescence images was possible. As silica beads were employed as artificial markers, the lack of inherent continuous structures which normally serve for the image registration in electron-microscopy, could be compensated. Hence, a rendered plastic image with an all-over nanoscopic resolution in three spatial directions could be representatively generated for synaptophysin and syntaxin1. The transfer of the technique to any other fluorescently labeled structure is thus enabled, independent of its continuous appearance in adjacent layers.

\section{Part C-Quantitative localization analysis of synaptic proteins}

Neuronal communication at chemical synapses relies on the $\mathrm{Ca}^{2+}$ triggered fusion of synaptic vesicles with the plasma membrane. Those vesicle carry a certain composition of neurotransmitters, which is released into the synaptic cleft and recognized by appropriate receptors on the side of neighbored dendritic spines. The fusion of synaptic vesicles is mediated by the so called soluble $N$-ethylmaleimide-sensitive-factor attachment receptor (SNARE) proteins, which form the in Fig. 3.14, B shown SNARE complex [33, 91]. The latter consists of SNAP-25 and synataxin1 provided by the plasma membrane, and synaptobrevin which is located on the vesicle surface. Thus, one would expect a high concentration of synataxin1 and SNAP-25 at synaptic sites, but a fairly low concentration in the peripheral neuronal membrane. So far it is known from the investigation of flat membrane sheds that both proteins form clusters within the plasma membrane [181]. To which extent the clusters are localized on synaptic sites, meaning in close proximity to the synaptic vesicles, remained unclear.

Hence, this question is now addressed with the explored approach of mechanical ultrathin sectioning in combination with two-color STED nanoscopy. To this end, synaptic vesicle sites are localized by immuno-staining of synaptophysin, which represents a wellknown marker for synaptic vesicles [92].

\section{Methods}

To investigate the distribution of the two synaptic membrane proteins synataxin 1 and SNAP-25 respectively, the proteins were immuno-stained with ATTO532. The correlation of the two proteins with the position of synaptic vesicles was realized by a co-staining of synaptophysin with a second color (ATTO633). The further sample preparation and imaging was performed analog to the previous experiments described in Part A of this Section.

For six images, the position of the protein clusters were marked manually in three independent runs, providing consistent position data. The size of the synaptic membrane clusters was subsequently determined in terms of the FWHM of the fluorescent spots.

Vesicle release sites were then identified based on the red detection channel for synaptophysin. A map of these regions was created using an adaptive thresholding algorithm implemented in MATLAB. In detail the script is ednoted in the Appendix. It preformed the following image processing steps: At first, regions below a noise level of 50 counts/pixel 


\section{Experiments and results}

were discarded, while the remaining regions were thresholded at their average intensity. The resulting masks were stable with respect to variations of the background level. Second, the masks were eroded with a radius $r=80-100 \mathrm{~nm}$ to exclude remaining artifacts and then dilated with a disk with $r=260 \mathrm{~nm}$ to account for the diameter of the vesicles. An example of a created mask is shown in Fig. 3.21. Clusters (represented as yellow stars in Fig. 3.21 , C) lying within the resulting masks were considered as "inside".

\section{Results and discussion}

The first result revealed by high resolution imaging of ultrathin sections was that both syntaxin1 and SNAP-25 showed a spotty distribution. The single clusters of synataxin1 and SNAP-25 can be separated from each other and individually (co-)localized within each section providing the imaging plane. The cluster size for the two membrane proteins was measured and is illustrated in Fig. 3.20. Clusters of SNAP-25 seem to be slightly smaller in diameter (100 nm in average) than those of synataxin1 (120 nm in average).

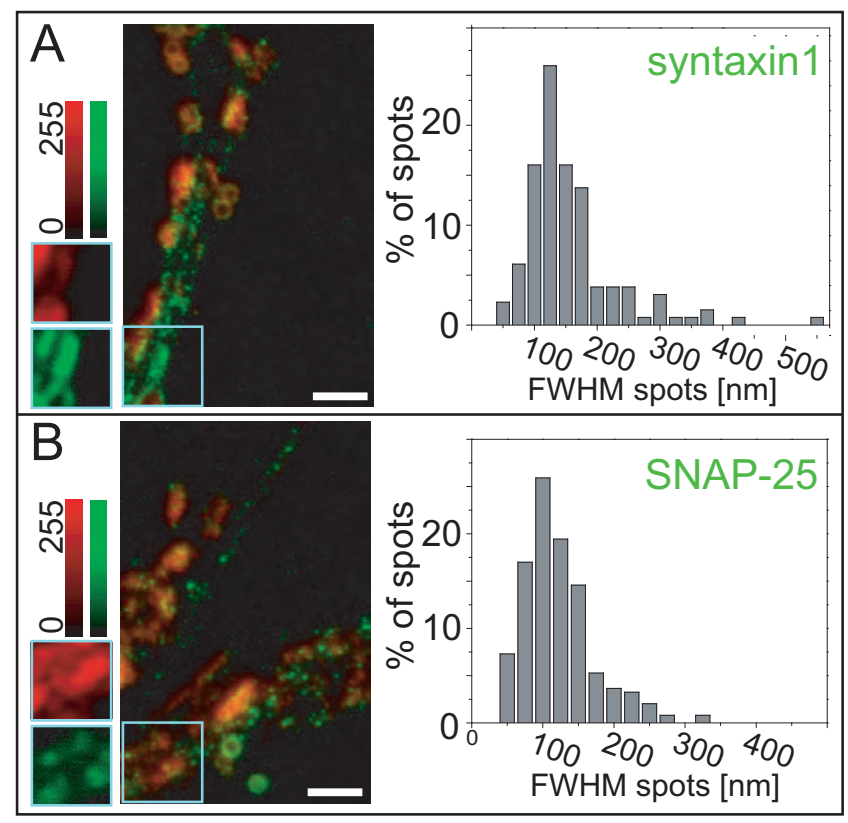

Figure 3.20: STED images and analysis of the cluster size distribution of two SNARE proteins after resin embedding and mechanical ultrathin sectioning. Shown are two color STED images of syntaxin1 (A) and SNAP-25 (B), both in green, co-stained with the synaptic vesicle marker synaptophysin (red). Scale bar: $1 \mu \mathrm{m}$. For the region represented by the white square the confocal images are additionally displayed for both color channels separately on the left. The spotty distribution of both SNARE proteins suggests the presence of numerous clusters of the proteins. A histogram of the measured cluster size is also shown for the two proteins. The size of most clusters was about $100 \mathrm{~nm}$ and $120 \mathrm{~nm}$ for SNAP-25 and syntaxin1 respectively. 
The spots of synataxin1 and SNAP-25 in the green channel largely surrounded the groups of synaptic vesicles (red channel), which are found within the axons. This is not surprising as both proteins are mainly found in the membrane of the axons. Some fluorescence was also associated with the synaptic vesicles sites, as both syntaxin1 and SNAP-25, despite being primarily plasma membrane proteins, are found at low levels in synaptic vesicles [168]. This type of staining would have been very difficult to observe via conventional confocal imaging due to the small size of the synaptic sites. Even with the enhanced lateral resolution of a STED microscope, the density of the synataxin1 and SNAP-25 staining obscured the individual spots. Thus clustering had so far only been observed by imaging membrane patches but not on a whole cell [155]. Here it is validated that the improvement of the axial resolution provided by the ultrathin sectioning protocol helps to investigate the distribution of the synaptic proteins.

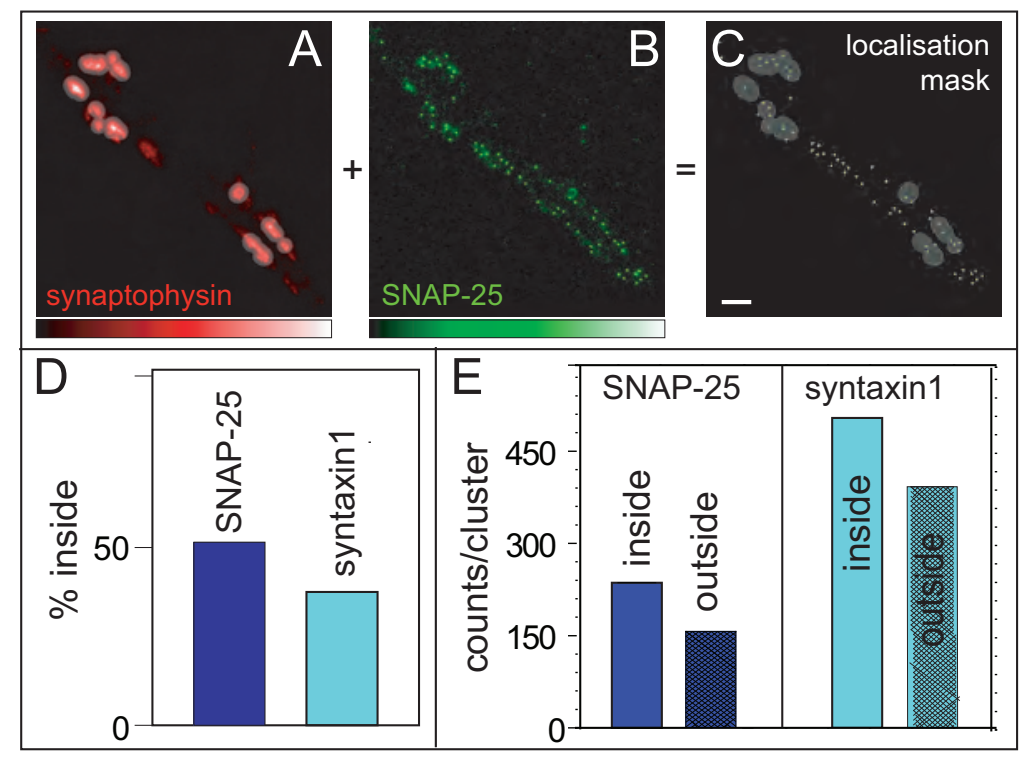

Figure 3.21: Analysis of the distribution of syntaxin1 (not shown) and SNAP-25 with respect to the colocalization with synaptic vesicles represented by synaptophysin after resin-embedding, mechanical ultrathin sectioning and STED imaging. Vesicle release sites were identified using an adaptive thresholding algorithm (gray regions in (A) and (C)) whereas clusters of SNAP-25 and synataxin1 were localized manually (yellow dots in (B) and (C). The cluster positions were classified according to whether they were localized within the identified release sites or not. (C) shows the localization mask derived from the STED images in (A) and (B). Scale bar: $1.25 \mu \mathrm{m}$ (D) shows the fraction of SNAP-25 and synataxin1 clusters occurring in vesicle release sites. In (E) the mean fluorescence counts for individual clusters of the two SNARE proteins inside and outside of the designed vesicle release sites are mapped.

As syntaxin1 and SNAP-25 function exclusively in vesicle fusion, one would expect 


\section{Experiments and results}

them to be concentrated in small areas of the synapse, in the release sites where synaptic vesicle fusion actually takes place. However, this is not the case: the two proteins are distributed in spots (protein clusters) across the entire sample. To confirm this, the percentage of SNAP-25 and synataxin1 clusters in the immediate vicinity of the areas occupied by the synaptic vesicles (as identified by the synaptophysin staining) was determined. It turned out that only $\approx 40 \%$ and $\approx 50 \%$ of the clusters of syntaxin 1 and SNAP-25 respectively are associated with synaptic vesicles. By varying the image processing parameters, these numbers were found to be stable to within $10 \%$. Finally, a small difference between the intensity characteristics of the protein clusters in areas occupied or not by the vesicles is found. In areas occupied by the vesicles, the signal seems to be slightly brighter which might also be due to a higher background in these regions. This indicates that there is no substantial difference between syntaxin1 or SNAP-25 clusters in the vicinity of the synaptic vesicles and those from other areas of the axons.

Overall, one is forced to conclude that syntaxin1 and SNAP-25, although functioning mainly in synaptic vesicle fusion, are not restricted to the space near synaptic vesicle accumulations. Interestingly, the wide positioning of the synaptic vesicle's fusion partners opens, in principle, the possibility of vesicles fusing outside of their normal release sites, in regions where neurotransmitter release would be unproductive. Thus it would not result in any transmission of information to the post-synaptic cell due to the lack of post-synaptic receptors (normally found only near the release sites). Again it is worth pointing out that this information would have been obscured by the out-of focus signal without applying ultrathin sectioning before imaging.

\section{Part D-Concerted investigation of recently endocytosed synaptic vesicles unconnected to the plasma membrane}

Whereas the $\mathrm{C} a^{2+}$-triggered exocytosis of synaptic vesicles is well accepted as the crucial step in intercellular communication due to neurotransmitter release, the fate of the synaptic vesicles after membrane-fusion is still heavily discussed [165, 142]. The three debated models for the process are "kiss-and-stay" (localized refill of a subpopulation of synaptic vesicles at the active zone), "kiss-and-run" (synaptic recycling is not restricted to the active zone, but goes rapidly without a Cathrin-coated intermediate) and "endosomalrecycling" (after Cathrin-coating, a certain fraction of the endocytosed vesicles is recycled via fusion with endosomes and subsequent budding, while others are simply refilled after uncoating). An overview of the three models is given in Fig.3.14. While the "kiss-andrun" model has received substantial attention in the last decade [142], the more complex full collapse and fusion pathway (endosomal-recycling) has been less actively investigated.

The endosomal sorting pathway was suggested in 1973 for the first time [76], although it turned out a few years later that it was based on erroneous interpretations [43]. The claim was heavily discussed in the following more than 20 years due to the lack of further evidence [165, 140, 176, 143]. However, without endosomal involvement, one is forced to assume that a unique synaptic vesicle composition is maintained by a single sorting step at the plasma membrane via the Cathrin-associated machinery [96]. This is questionable, although it could be shown that different synaptic proteins remain clustered after fusion 
[179, 181], since only few proteins have the ability to directly interact with this machinery. Thus, even limited fragmentation upon fusion would imply that substantial sorting (for example via endosomes) would be required.

In experiments performed by Peer Hoopmann ${ }^{3}$ it could be shown that after exocytosis synaptic vesicle components undergo limited spreading throughout the plasma membrane, which must consequently be followed by retrieving imperfect vesicles with an inconsistent protein composition. Those imperfect vesicles are rather likely to contain impure membrane fragments, which were incorporated in addition to synaptic vesicle components.

To verify this prediction, one has to deal with the problem to select a distinct thin layer of a sample, which is less than $100 \mathrm{~nm}$ thin. A confocal focus axially extends to at least $500 \mathrm{~nm}$ and thus does not allow an ultrathin layer to be selectively addressed. However, a separation between proteins either found within the neuronal membrane, at a certain distance to the membrane is at this point strongly required. Therefore ultrathin sectioning is essential in this respect. The strategy is visualized in Fig. 3.22, A. The size of the confocal focus is indicated by the grayish shadow, while the attention should be turned on it's axial extension in particular. In the following it is described, that ultrathin sectioning allowed a specific investigation of distinct layers within a neuronal axon including the unambiguous identification of vesicle clusters void of plasma membrane.

\section{Methods}

Neuronal cultures were stimulated for endocytosis and immuno-stained against synaptophysin with ATTO532, and for two membrane-resident proteins, syntaxin1 and SNAP-25, with ATTO647N. The samples were resin embedded into Nanoplast and cut into roughly $80 \mathrm{~nm}$ thick sections. The samples were embedded in Moviol an d afterwards invetigated. As synaptic vesicles can be estimated to be larger than the signal obtained from fluorescently labeled synaptophysin, a confocal resolution in the lateral direction was assumed to be sufficient in the visible channel. For the detection of the red channel (syntaxin1 or SNAP-25), a high resolution was favored. A commercial TCS STED microscope (Leica Microsystems GmbH, Mannheim, Germany) providing one high resolution channel in the infrared region (with a resolution $>70 \mathrm{~nm}$ ) and, in addition, confocal detection channels were used.

\section{Results and discussion}

According to the hypothesis, one could detect a significant increase in colocalization of membrane clusters with synaptic vesicles compared to the unstimulated case. Colocalized SNAP-25 and synaptophysin is indicated by yellow areas in Fig. 3.22, B. To visualize what is observed in either case the results are additionally sketched next to the images. After stimulating the endocytosis of synaptic vesicles, a significantly increased amount of the labeled membrane proteins can be found in close proximity of the synaptic vesicles marked by synaptophysin.

\footnotetext{
${ }^{3}$ (European Neuroscience Institute, Göttingen, Germany).
} 


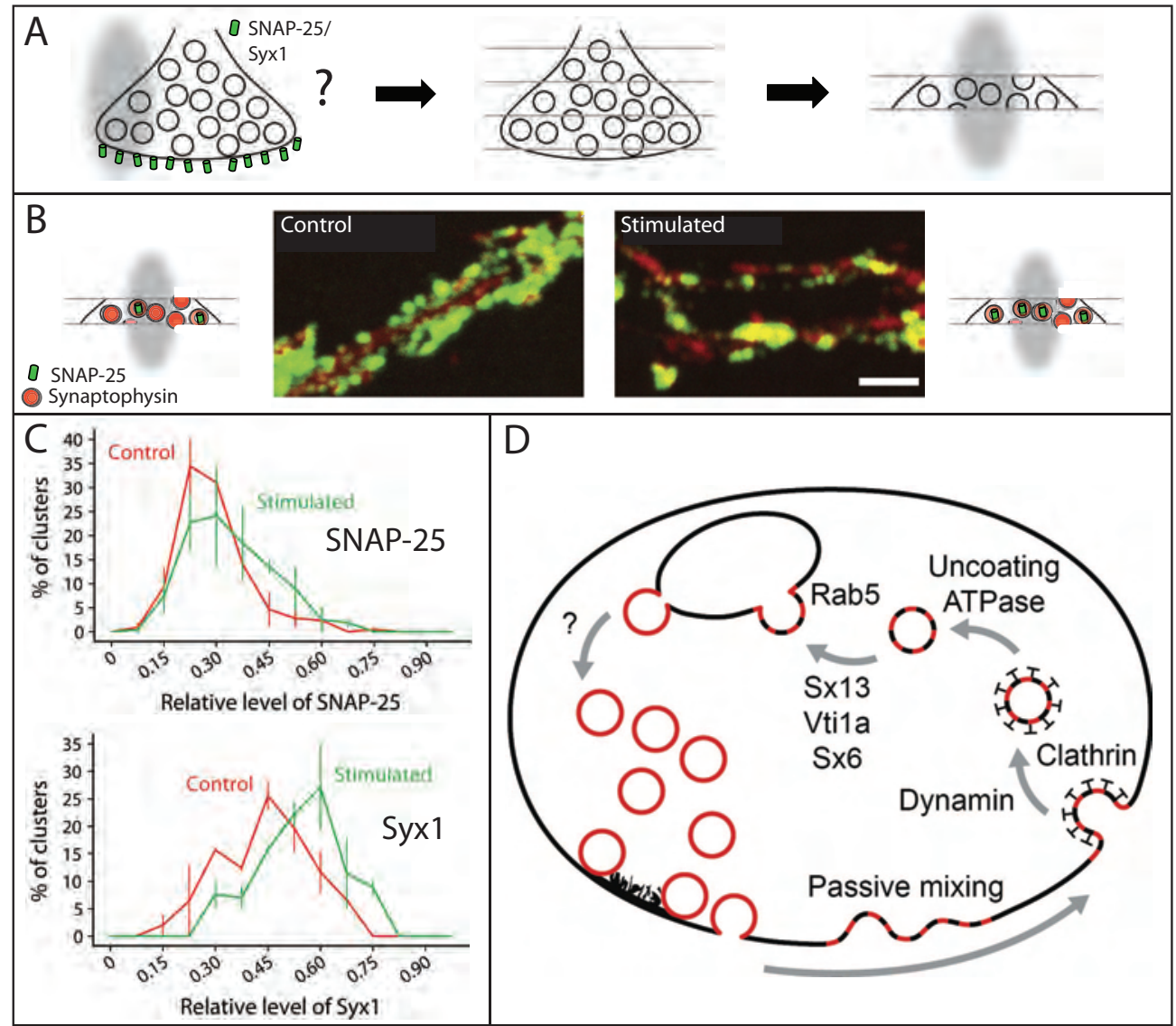

Figure 3.22: A: Schematic depiction of the work flow. Using a confocal focus in axial direction does not allow the distinction between SNAP-25 and Syntaxin1 located in the membrane or in the synaptic vesicles. Sectioning and subsequent imaging of slices allows selective imaging of the vesicles. B: Control or stimulated hippocampal cultures with SNAP-25 (green, confocal) and synaptophysin (red, STED) immunologically stained. Note the increase of signal colocalization after stimulation; section thickness: $80 \mathrm{~nm}$; scale bar: $2 \mu \mathrm{m}$. C: Quantification of the SNAP-25 (top, average of 3 independent experiments) and syntaxin1 (bottom, average of 2 independent experiments) intensity within the vesicle clusters. D: Sketch visualizing the concluding results of the current study: endosomal recycling is true for at least a part of endocytosed synaptic vesicles; for details see text.

The quantitative analysis of the relative level of each of either synataxin1 or SNAP25 within the synaptic vesicles underscores this findings: The according data analysis was performed by picking the centers of large vesicle clusters. Subsequently a region of interest with a diameter of $50 \mathrm{~nm}$ was selected around them and the average fluorescence for both the synaptophysin and the membrane protein channel was calculated. For a clearer view on the data, the fluorescence in the SNAP-25 or syntaxin1 channel relative 
to that of synaptophysin was determined and plotted as a histogram $(3.22$. C). The levels of both SNAP-25 and syntaxin1 in the vicinity of synaptic vesicles was increased after stimulated endocytosis. Thus, it was substantiated that fragments of the neuronal membrane had been incorporated into the recently formed vesicles.

Further experiments performed by Peer Hoopmann showed that afterwards at least a part of those "imperfect" vesicles fuse with endosomes since they showed a markedly enrichment of previously labeled synaptic vesicle proteins. Moreover, new vesicles seem to bud again from the endosome within $30 \mathrm{~s}$. Hence, this indicated that they undergo an endosomal recycling, while others might follow the faster "kiss-and-run" model. Perhaps vesicles perform repeated kiss-and-run fusion followed by endosomal recycling whenever "imperfect" kissing results in partial loss of the identity in the plasma membrane [188]. However, the detailed mechanism for the endosomal budding of vesicles as the last step in the recycling cycle still remains unknown at this point. 


\subsection{Application II: Nanoscopy on fluorescent fusion proteins in C. elegans}

A discipline, which can substantially profit from optical nanoscopy on ultrathin sections is the investigation of the nematode Caenorhabditis elegans (C. elegans). It is currently one of the most important organisms in the field of developmental biology and for genetically studies. Foremost employed for laboratory investigation by Sydney Brenner in the late 1960's [17], it was the first multi-cellular eukaryotic organism to have its genome completely sequenced in 1998 [82]. The small animal, which is about $1 \mathrm{~mm}$ long and has a diameter of about $36 \mu \mathrm{m}$ is studied as a model organism for a variety of reasons. Figure 3.23 gives an idea of the lucent animal and it's organs. Beside the fact, that it's rapid and easy to bread, it's structural simplicity, including the cell constancy, are expedient qualities in research. Further interest rose from it's clearly arranged nervous system, consisting of only 302 neurons. It serve as a model organism and many results from developmental and genetically studies can be passed on vertebrates, including humans.

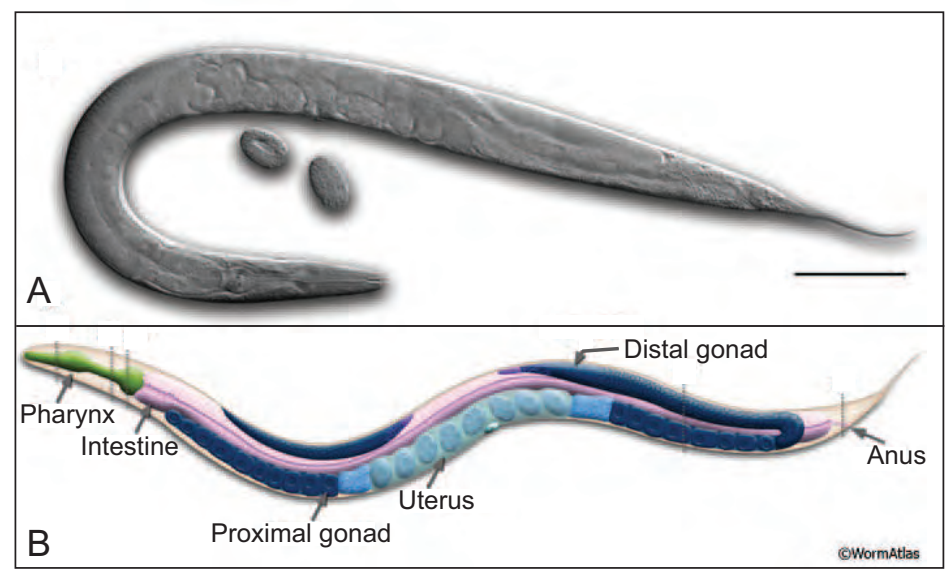

Figure 3.23: Anatomy of an adult hermaphrodite of C. elegans. A: DIC image of an adult hermaphrodite, left lateral side. Scale bar $0.1 \mathrm{~mm}$. B. Schematic drawing of anatomical structures, left lateral side. 84.

After resin embedding and ultrathin sectioning, C. elegans has been extensively studied by means of transmission electron-microscopy. Much of the available information is documented in the wormatlas, a library of pictures of nearly any part of the worm 84. Anyway addressing a particular biological problem by means of microscopy requires sensitive markers. For distressing manner, on whole animals like C. elegans, staining with specific antibodies is often hindered. The labels, if available at all, are mostly membrane-impermeable so that an effective labeling of proteins or peptides is not or only insufficiently assured.

However, C. elegans was the first organism, which was successfully transfected with the green fluorescent protein GFP [21]. Because of the simple genome of C. elegans, protein modifications are nowadays easily generable. The genetically encoding with fluo- 
rescent proteins (FPs) ends up in an outright fluorescent labeling of a certain structure. In consequence, this prompted numerous selective light-microscopic studies on different proteins, even in the living animal [132, 54, 52, 81]. Still, fluorescent proteins have their own drawbacks. As they are quite large, especially if they are only available as oligomers, they might cause structural artifacts. Furthermore, the genetically encoding might markedly influence the cellular process in an uncontrollable way. A major disadvantage for microscopic studies is their low photostability.

During the imaging of FPs, the resolution of a confocal fluorescence microscope is not sufficient for many questions. Unambiguous information about the location or the distribution of the labeled proteins can thus not be obtained. Although fundamental progress has recently been made in the field of high resolution light microscopic techniques, semi-thick samples (e.g. worms) with densely labeled structures are still not entirely addressable. For STED microscopy, the limitations are mainly related to photobleaching, in particular regarding the imaging of fluorescent proteins.

By mechanical slicing of the sample, photobleaching is restricted to the investigated layer only. Hence, genetically labeled proteins can be imaged with nanoscopic resolution in each single layer of the sample. Moreover, correlative STED and EM can combine the ultrastructure and protein-specific high-resolution images of the same tissue section. Still, FPs, although shielded by the beta barrel, are more sensitive to environmental changes than are organic fluorophores. Though the use of FPs together with resin embedding for ultrathin sectioning is a special challenge.

Primarily due to its photostability the most suitable currently available FP for STED microscopy was shown to be Citrine [64]. Citrine is a variant of the yellow fluorescent protein (YFP) with a similar absorption and emission spectrum but higher resistance against environmental changes, e.g. $\mathrm{pH}$ [62. A resolution enhancement in either the axial or lateral direction could be achieved by STED microscopy on cultured living cells expressing Citrine. Still, a simultaneous three dimensional investigation of semi-thick samples with sub-diffractional resolution has not been shown yet. Each scanned optical section by the STED beam goes along with a destructive photobleaching of the fluorescent structures in the peripheral layers. Thus, until more resistant FPs are available, mechanical slicing after resin embedding is the method of choice to investigate different layers of the worm with the nanoscopic resolution of STED microscopy.

In this study, two different proteins were tagged with Citrine and subsequently expressed in C. elegans. 者

The first protein, which was observed is synaptogyrin1 (sng1) that occurs on the membrane of synaptic vesicles. The importance of studies related to neuronal communication and the synaptic vesicle cycle was already outlined in Section 3.3. The synaptic protein synaptogyrin1 was chosen, since it is one of the few unambiguous synaptic vesicle markers [60, 132]. Whereas proteins like snb1 are not only expressed within the vesicles but also in the neuronal membrane, sng1 is only tightly localized on the vesicles themselves.

\footnotetext{
${ }^{4}$ The project was set up as a collaboration with the lab of Erik Jorgensen, in particular Shigeki Watanabe, at the University of Utah. In detail, breading, embedding and partly the sectioning of the worms, as well as electron-microscopic imaging was done in the Jorgensen lab, while other sectioning, further processing of the sections and fluorescence imaging including STED was done by me.
} 


\section{Experiments and results}

In Fig. 3.24, A the course of neurons within C. elegans is sketched. Additionally a fluorescence widefield image of sng1 is shown. The (cross-) section was taken far from the nerve ring, the worms brain. The two main nerve cords, the ventral and dorsal ones, are indicated.

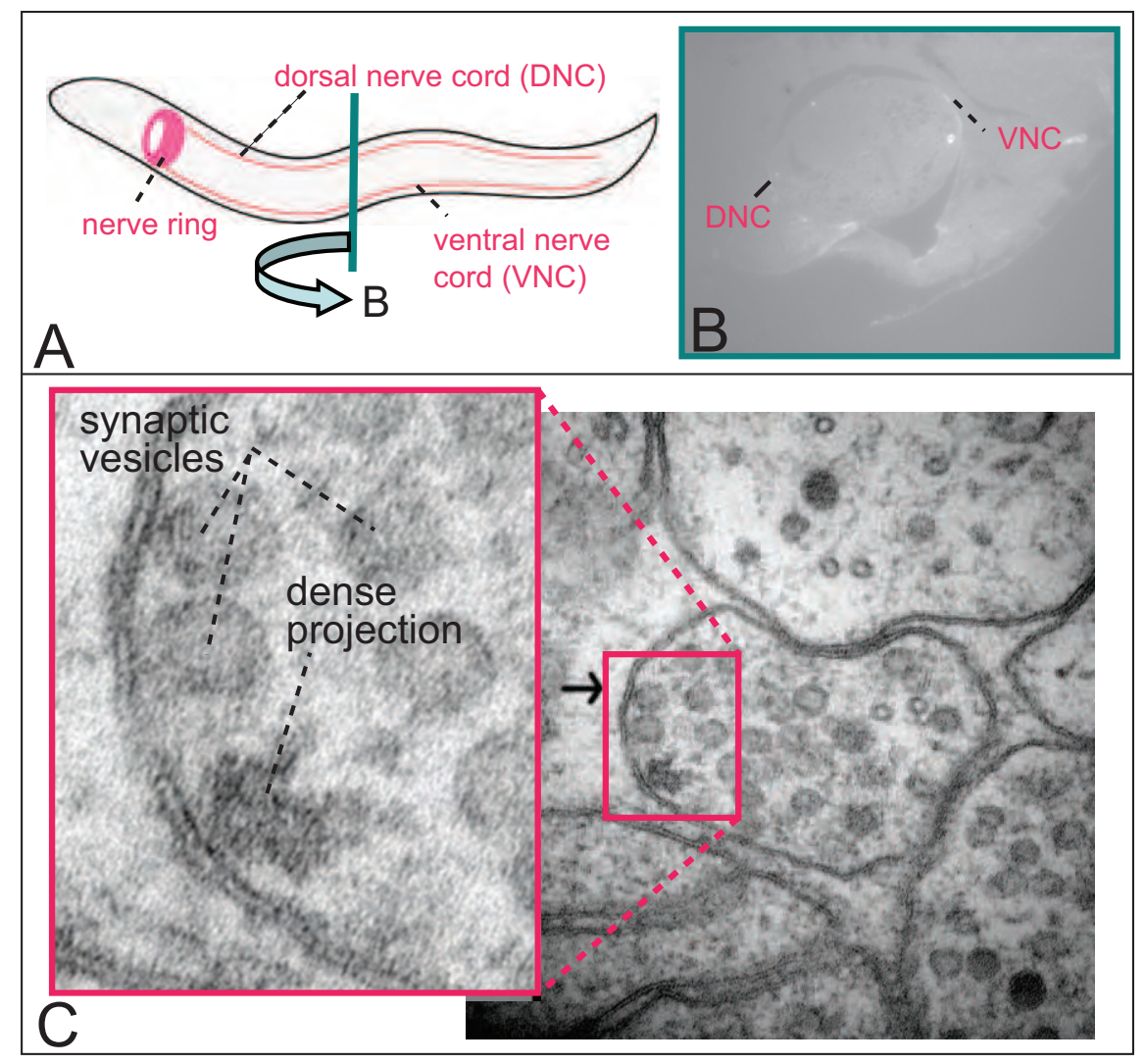

Figure 3.24: A: Neuronal sites within C. elegans, where sng1 is expressed.[85] B: Widefield fluorescence image of a crossection of $C$. elegans expressing sng1 together with Citrine. C: electron-microscopic picture of a nerve. The enlargement shows synaptic vesicles surrounding an active site or dense projection at the synapse.

Although synaptic vesicles, as well as the active sites, are easily recognizable on EM images, specific synaptic processes are hard to study with this method. Active sites, which consist of cadherines and transmitter docking proteins, are very densely packed and therefore also called dense projections. An example for an image of an active site, surrounded by several synaptic vesicles is shown in Fig.3.24. Ultrastructural details including membranes are resolved with an impressive resolution. Unlike, the localization of single distinct proteins is not or only to a very limited extend, possible. High resolution methods, enabling the specific proteins to be addressed were for a long time lacking. Now, several fluorescence techniques have been established, among them STED microscopy. 
Bringing those methods together with the ultrastructural resolution of EM is obvious. Thus, alternatively to two-color imaging, as it was performed in Section 3.3. correlative investigation of the same section by STED and electron-microscopy, followed by superposition of the resulting images can help to precisely localize a certain protein related to synaptic vesicles. This will help to understand the proteinogenic background of cellular processes in general and at synaptic sites in particular.

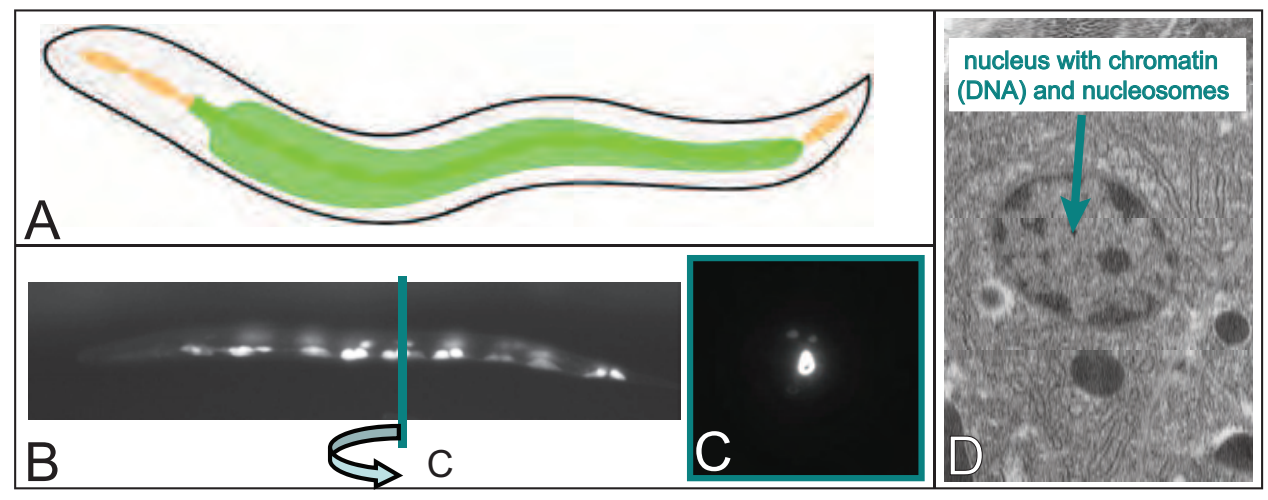

Figure 3.25: A: The sites where his44 is naturally expressed are indicated in green. [85]B: Fluorescence image of $C$. elegans expressing his44 together with GFP. C: Widefield fluorescence image of a cross section of an animal, where his44 is genetically encoded with Citrine. D: TEM image of a section of C. elegans. Densely packed cell nuclei, where chromatin and therewith histones are localized, are clearly visible as dark structures and indicated. [84]

In a second experiment, Citrine was expressed together with a histone. Histones are the core proteins of nucleosomes in eukaryotic cells. A single nucleosome consists of an octamer of eight histones, following the nomenclature in vertebrates, two of H2A, H2B, $\mathrm{H} 3$ and H4. The DNA is about 1.65 times wrapped around a single nucleosome, which is equivalent to about 146 base pairs. The for those studies selected histone his44 is a homologous to the protein $\mathrm{H} 2 \mathrm{~B}$ in vertebrates. An fluorescence widefield image of a worm expressing his44 together with GFP is shown in Fig.3.25. B. A crossection is given in C. It is naturally only expressed in the intestinal cells of C. elegans (see Fig. 3.25, A), while in this study, the promoter was selected such, that it is expressed in the nucleus of any cell of the animal.

As shown in Fig. 3.25, D, tightly DNA packaging around the nucleosome is clearly visible in the EM pictures. However, it is desirable to learn more details of the mechanisms, which are responsible for the DNA packaging. By high resolution imaging, for example with STED nanoscopy, further investigation of the localization and therewith the function of specific proteins is generally enabled. In particular densely packed nuclear structures are revealed by ultrathin sectioning and the accompanied axial resolution refinement. Again, the correlation of STED data with EM images of the same ultrathin section enables the localization of a specifically labeled structure with the cellular ultrastructure 


\section{Experiments and results}

of the worm.

The following experiments show, that it is possible to conserve the fluorescence signal of Citrine during fixation and resin embedding. After ultrathin sectioning it still yields enough signal to obtain high quality STED images. In addition, correlative STED an electron-microscopic study is performed for the case of his44.

\section{Methods}

The expression of the investigated fluorescent fusion proteins was performed according to the methods described elsewhere [122, 40]. Briefly the modified DNA constructs are introduced into the gonads of an adult hermaphrodite by micro-injection.

Because of their size, specimen of $C$. elegans are well suited for high pressure freezing methods (HPF). For small samples this is currently the method of choice to highly ensure a preservation of ultrastructural information. Rapidly applying a pressure of about 2000 bar or more to specimen kept in their natural environment, e.g. a bacteria solution, in combination with cooling to $-173^{\circ} \mathrm{C}$ within less than $500 \mathrm{~ms}$, suppresses the formation and growth of ice crystals. Instead, vitreous ice is formed and the ultrastructure of the sample is mostly retained. For HPF a Baltec HPM 010 machine was employed. A comparison between HPF and conventional aldehyde fixation methods at room temperature is given in Fig. 3.26, showing the improved structure preservation in HPF. The electronmicroscopic pictures show the dorsal nerve cord of C. elegans at a magnification of $135 \mathrm{k}$.

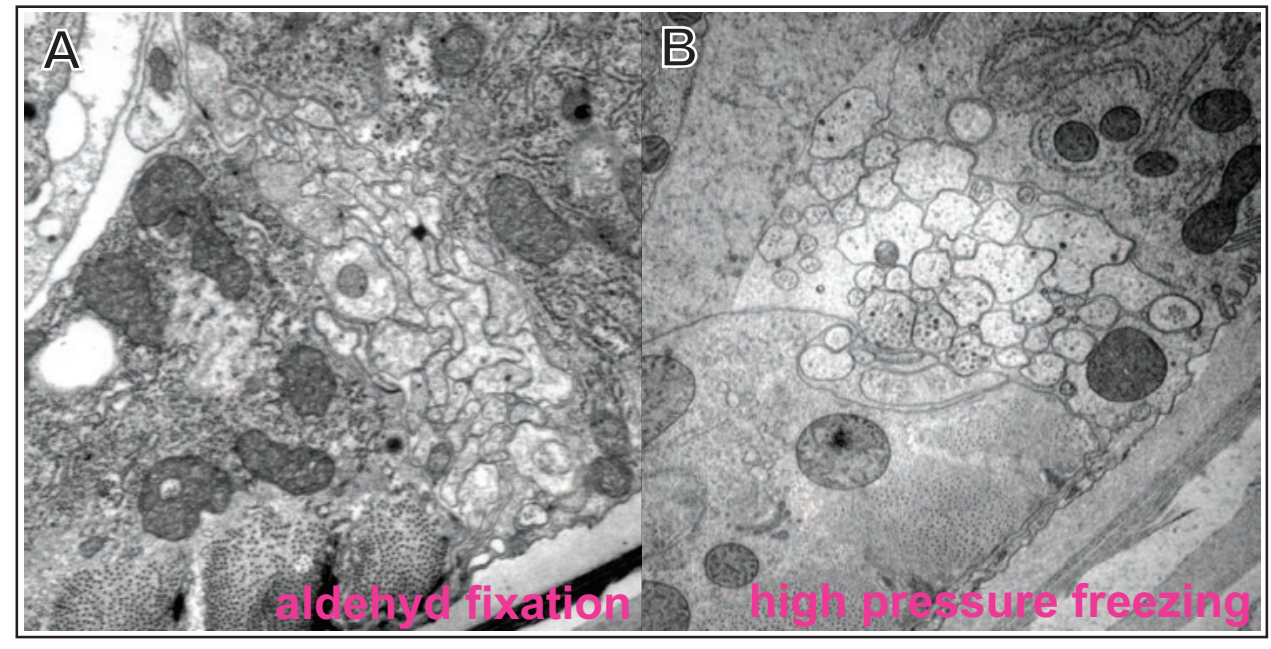

Figure 3.26: Comparison of a TEM image of the dorsal nerve cord of C. elegans after aldehyde fixation (A) or HPF (B). The ultrastructure is clearly better preserved in the latter case.

The further treatment of the sample is dominated by balancing out the requirements of electron-microscopy on the one hand and fluorescence microscopy on the other hand. EM calls for a strong fixation of ultrastructural details and hence, a proper throughout 
polymerization of the resin. Fluorescence microscopy, however, demands the conservation of the signal from the expressed fluorescent proteins. This includes the mandatory presence of a certain amount of water, since the chromophore is highly dependent on the coordinated hydrogen bonding network. Complete dehydration of the samples has to be avoided.

Finally it turned out, that a fixation with $0.1 \% \mathrm{KMnO}_{4}$ is the most gentle way to treat the sample while meeting all mentioned demands. The fixative is solved in acetone, which remains liquid at low temperatures. The ice substitution after freezing by the fixative and subsequent infiltration steps with the monomer solutions are performed in an automated freeze substitution unit (Leica AFS2). First the specimen is stepwise thawed from $-90^{\circ} \mathrm{C}$ to $-20^{\circ} \mathrm{C}$ and afterwards kept at this temperature until polymerization was completed. What turned out to be important is that throughout the whole treatment a content of $5 \%$ water has to be present. In addition to the demands of the FP, it assures a neutral $\mathrm{pH}$ during polymerization. The polymerization itself is performed by an accelerator, whose concentration increased with every infiltration step.

Finally polymerized blocks are thawed, trimmed and cut into ultrathin sections of $70-100 \mathrm{~nm}$ in thickness. The sections were transferred to either blank coverslips. The sections are sensitive to oxygen, but can be stored at $-20^{\circ} \mathrm{C}$ for several weeks after applying nitrogen and subsequent evacuation. This enabled also the shipment from Utah to Göttingen without a loss of the fluorescent signal.

For STED imaging the samples were thawed and incubated with a aqueous solution of fluorescent silica beads (sicastar blueF, micromod, Germany). With the STED setup they can be excited and detected separately from the proteinogenic fluorescence recording an additional UV image. In the electron-microscope they are also clearly visible because of their comparatively high density. This helped to finally superpose the fluorescence images with electron microscopic pictures.

After evaporation of the water at room temperature, the sections were mounted in distilled water and imaged with the STED microscope described in [64]. Instead of stage scanning, the image acquisition was performed with a beam scanning mirror similar to the one described by Westphal et al [177]. In brief, the excitation pulses were delivered by a pulsed laser diode (Toptica) at a wavelength of $488 \mathrm{~nm}$ and a pulse duration of $100 \mathrm{ps}$. The light was focused into a objective lens (PL APO, 100x/1.4 NA, oil, Leica). The fluorescence signal was collected by the same lens. It was separated from the excitation light by a custom-made dichroic mirror and filtered with a 560/20 bandpass filter. A multimode fiber served as a confocal pinhole. The pulsed STED light was derived from a Ti:Sapphire laser (MaiTai, Spectra-Physics) operating at $80 \mathrm{MHz}$ and emitting at $795 \mathrm{~nm}$. The $200 \mathrm{fs}$ pulses were stretched to $300 \mathrm{ps}$ by by dispersion in a polarization-preserving fiber. The excitation pulses were synchronized to the STED pulses by external triggering of the laser diode. The pulse delay was generated by a home-build delay generator. The image size was typically chosen to be $20 \mu \mathrm{m} \times 20 \mu \mathrm{m}$ with a pixel size of $30 \mathrm{~nm}$ and a pixel dwell time of $10 \mu \mathrm{s}$. The total detection time for one image was in the range of a few minutes. To begin with a confocal image was detected with an excitation power of $1 \mu \mathrm{W}$ in the aperture. Afterwards the STED imaging was performed with a higher excitation power of about $12 \mu \mathrm{W}$ and a STED beam power of $45 \mathrm{~mW}$ in the aperture. To detect 


\section{Experiments and results}

as much signal as possible $2-3$ scans were performed for STED imaging and added up. Finally, when all fluorescence was bleached, a confocal UV image was detected by an additional scan of the same section. The excitation was performed with a pulsed photodiode working at $405 \mathrm{~nm}$ (Oxxius S.A., Lannion, France).

After STED imaging the sections were dried on air and sent back to Utah. There the slices were imaged by SEM. To this end, an FEI nova nano with VCD detector for back-scattered electron detection was applied at low vacuum. The acceleration voltage was $5 \mathrm{kV}$.

\section{Results}

Because FPs are sensitive to oxidation by atmospheric oxygen, there were so far only very few studies on resin embedded and ultrathin sectioned samples. Up to now only semithick samples of $0.5-1 \mu \mathrm{m}$ thickness were examined by confocal fluorescence microscopy [117, 99]. Confocal imaging implies a resolution limit of $500 \mathrm{~nm}$ in all three spatial directions or a cubic voxel extension.

\begin{tabular}{|c|c|c|c|}
\hline & electron-microscopy & $\begin{array}{c}\text { fluorescence- } \\
\text { microscopy with FPs }\end{array}$ & solution \\
\hline 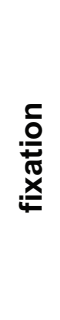 & $\begin{array}{l}\text { hard fixation to conserve } \\
\text { ultrastructure : } \\
\text { - } \text { GA fixation (less } \\
\text { structure } \\
\text { preservation) } \\
\text { - } \mathrm{OsO}_{4} \text { fixation }\end{array}$ & $\begin{array}{ll}\text { soft fixation to conserve } \\
\text { fluorescence: } \\
\text { - } \text { no } \\
\text { autofluorescence } \\
\text { (GA) } \\
\text { - no high amounts } \\
\text { of oxidizing agents } \\
\left(\mathrm{OsO}_{4}\right)\end{array}$ & $\begin{array}{c}0,1 \% \mathrm{KMnO}_{4} \\
\text { or } \\
0,01 \% \mathrm{OsO}_{4}\end{array}$ \\
\hline 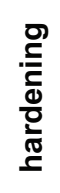 & $\begin{array}{ll}\text { - } & \text { acidic } \mathrm{pH} \\
\text { - } & \text { hydrophobic } \\
\text { environment, } \\
\text { dehydration }\end{array}$ & $\begin{array}{l}\text { - } \text { no acidic } \mathrm{pH} \\
\text { - } \text { crystal water }\end{array}$ & $\begin{array}{l}5 \% \text { water in LR } \\
\text { White during } \\
\text { infiltration and } \\
\text { polymerization } \\
\text { solves both issues }\end{array}$ \\
\hline
\end{tabular}

Table 3.1: Conflicting requirements of electron- and fluorescence- microscopy on resin embedded samples and elaborated solutions.

While high-resolution methods like STED microscopy paved the way for the observation of nanoscopic features labeled with FP in the lateral direction, the axial direction was the limiting factor. Thus, the call for even thinner slices arose. Incomplete dehydration procedure had been reported to introduce artifacts on the ultrastructure during ultrathin sectioning [156]. This is mainly because of the insufficient polymerization of the acrylate resin, which resulted from small amounts of water in the polymerization mixture. An alternative approach uses cryo-sections to circumvent at least the dehydration problem [137. 
A new protocol that preserves both, the ultrastructure of the sample and also the fluorescence of the FP, even in sections less than $100 \mathrm{~nm}$ thick is applied. An overview of the conflicting requirements in electron and fluorescence microscopy is given in Table 3.1 . Also, the elaborated solutions are mentioned. During the infiltration procedure with the monomeric resin solution, oxygen contact was reduced by storing the sample in an AFS unit. Moreover, the resin mixture was extricated from oxygen by placing it into a vacuum chamber after mixing. The fixation with potassium permanganate or a low concentration of osmium tetroxide saves the ultrastructure up to the final sectioning step, even if $5 \%$ of water are present. This amount of water is also conducive to lower the destructive effects of acidic sites within the resin on FPs. In the end, a well-defined signal from Citrine in ultrathin sections of LR White was observed and yielded enough photo-counts for high resolution STED imaging. However, for long-term storage, keeping the samples at $-20^{\circ} \mathrm{C}$ in bags, first filled with nitrogen and afterwards evacuated, is mandatory.

Synaptogyrin1 To validate nanoscopy on fluorescent fusion proteins in ultrathin sections, a fusion protein of Citrine and sng1 was expressed in C. elegans. After HPF and embedding, the animals were sectioned into $<100 \mathrm{~nm}$ thick slices, which were subsequently mounted on coverslips. After thawing the frozen sections, they were mounted in water and directly imaged with STED microscopy. Fig. 3.27 shows exemplary confocal and STED images of sng1 in a section through the nerve ring of C. elegans. Here, synapses and therewith synaptic vesicles are particularly frequent. It is clearly evident, that imaging of fluorescent proteins in plastic sections with high resolution is possible by means of STED microscopy.

As cellular structures are for the most part auto-fluorescent in the UV, recording a confocal image with an excitation of $405 \mathrm{~nm}$ reveals details of the peripheral structures in the animal. In Fig. 3.27, C, which is an overlay of the STED image with the according UV image, the pharynx in the middle of the nerve ring for instance is clearly visible. The additional information helps to put the high resolution protein image into a larger structural context. This is desirable for STED microscopy, since multicolor imaging mostly goes along with a more sophisticated setup. Imaging a single color is very often little meaningful. In addition a single color fluorescence image is in most cases little meaningful.

In Fig. 3.28 confocal and STED images of sng1 at a crossection of the backmost part of the animal at the ventral nerve cord is shown. In panel $\mathrm{C}$ also a line profile is plotted, which was measured for the in the STED image indicated site.

It substantiates, that structures can be resolved with a resolution below $50 \mathrm{~nm}$. This corresponds to the size of a single synaptic vesicle. As the image shows numerous regions, where the fluorescence signals can not clearly separated, it is likely, that several proteins of sng1 are found in each vesicle.

A substantial gain in information already results from the ultrathin sectioning of the sample. Especially for vesicles with a diameter of about $30-40 \mathrm{~nm}$, which are naturally tightly clustered, decreasing the thickness of the observed sample volume by a factor of five enables a much more precise localization [60]. 


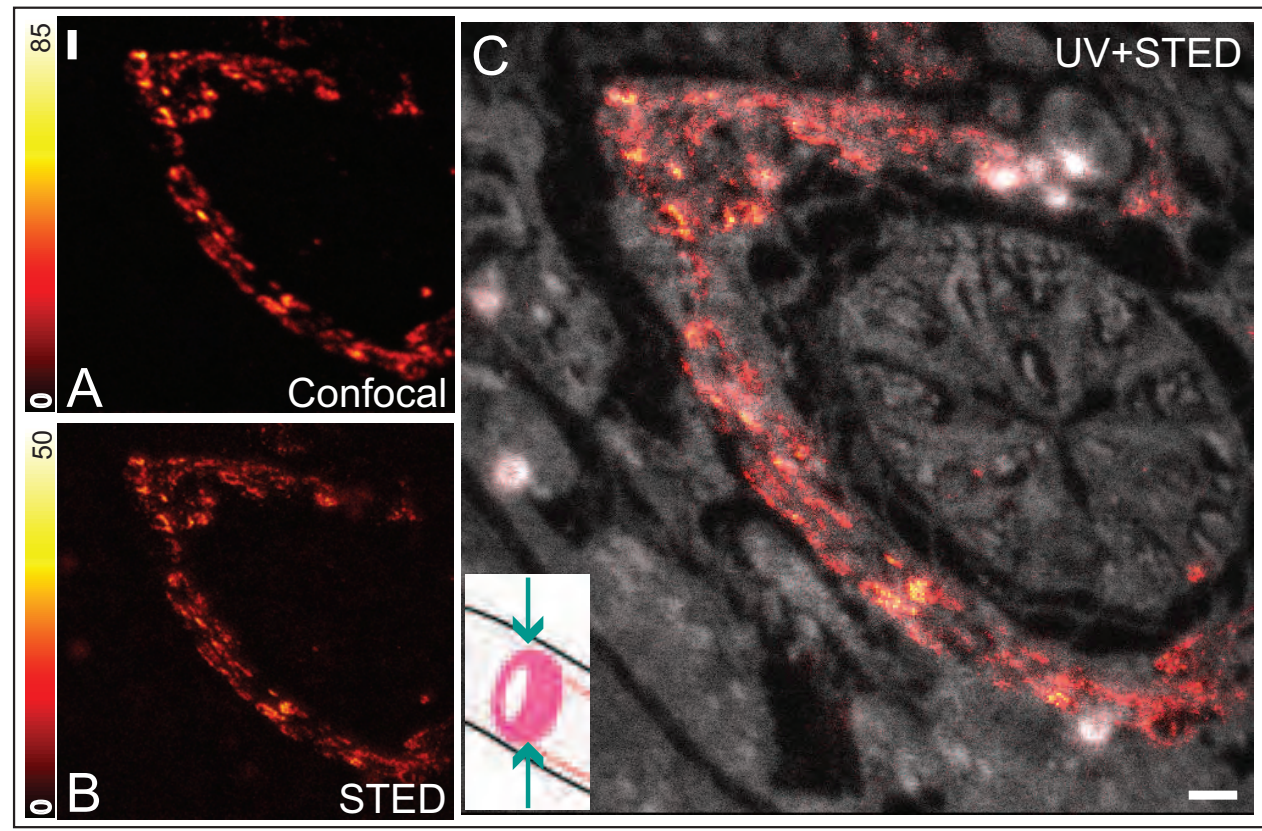

Figure 3.27: Confocal (A) and STED (B) image of Confocal (A) and STED (B) images of sng1 genetically encoded with Citrine in the nerve ring of $C$. elegans after resin embedding and ultrathin sectioning of the animal. C: Overlay of the STED image shown in B with the UV image of the same region, visualizing the structural context of the fluorescent structure. The inset is taken from the sketch in Fig. 3.24, A, to facilitate the orientation in the worm. Section thickness: $100 \mathrm{~nm}$. Scale bar: $1 \mu \mathrm{m}$.

Moreover, structures, which were previously smeared out by a worse SNR can be revealed on the ultrathin slices. However, an even more detailed information is provided by high resolution fluorescence images.

In future studies, correlating confocal or STED images with electron microscopic data can give further insight into the localization and distribution of sng1.

Histone44 Another protein, which was successfully studied with STED nanoscopy, is his44. A representative image is given in Fig. 3.29. Compared to the confocal image, where the substructure within the cell nucleus is more or less blurred, the chromatin network is at least rudimentarily discernible in the STED image. To study the function of a certain histone in the folding mechanisms of the DNA, modified histones, which might show an altered spatial assembly within the chromatin network, could be investigated with the technique.

To the end of correlative STED and SEM imaging on the nucleosomal protein his44, the fluorescent sections placed on coverslips were incubated with a solution of in the UV range fluorescent beads. Due to their polar surface, the silica beads tightly stick to the acrylate resin sections. They can serve as fiducial markers, as a UV image can easily be 
taken in the STED setup immediately after the acquisition of the high resolution image. Because scanning is performed by a beam scanning mirror, no drifts of the sample itself are to be expected either.

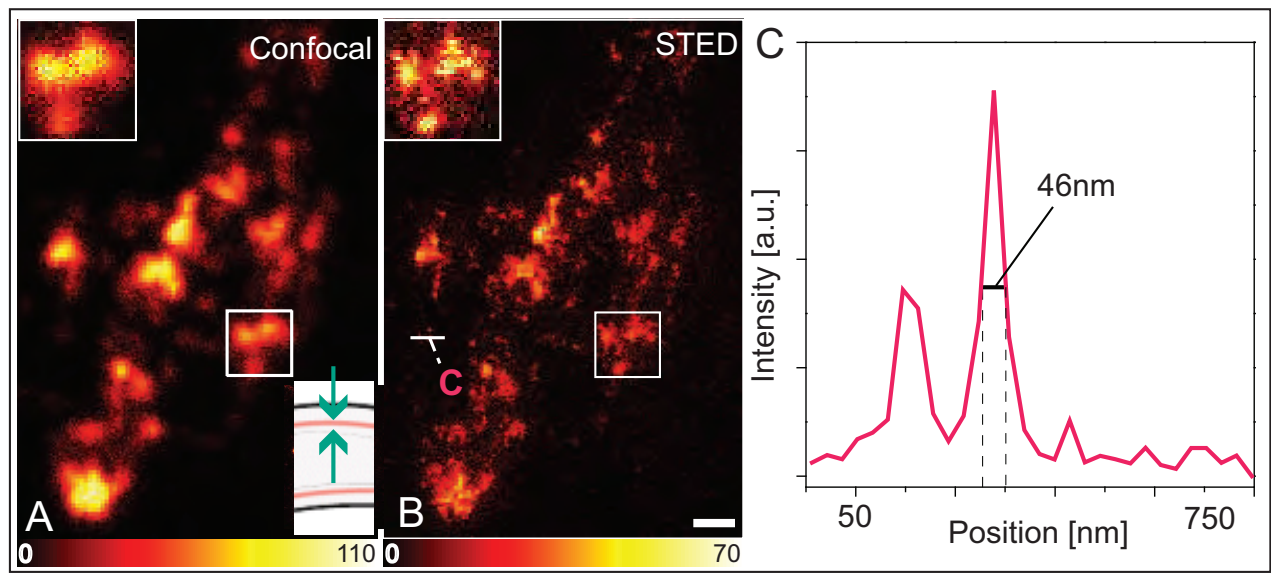

Figure 3.28: Confocal (A) and STED (B) images of sng1 genetically encoded with Citrine in the ventral nerve cord of $C$. elegans after resin embedding and ultrathin sectioning of the animal The insets show an enlargement of the regions indicated by the white square. A lineprofile of the in the STED image indicated position is also shown (C). Section thickness: $100 \mathrm{~nm}$. Scale bar: $1 \mu \mathrm{m}$.

Moreover, silica is a highly electron scattering material. Hence, a localization of the beads in the electron-microscopic picture is quite unambiguous. Although the chromatin structure itself facilitates the superposition of the data, fluorescent beads are even more incontestable. In addition, they allow the transfer of the technique to the investigation of any other genetically encoded fluorescent protein that is not easily identifiable in electronmicroscopic data. Figure 3.30 gives an overview of the work flow towards correlative STED-EM-imaging.

Figure 3.31 finally substantiates that correlated STED-EM-imaging works. However, the structures in the STED image are comparably dim, yielding in a decreased SNR in the images. This is also, because the section was cut to $75 \mathrm{~nm}$ only, which is about three-quarter of the previously in this Chapter discussed samples.

After deconvolving the STED image with an Richardson-Lucy it was superpositioned with the according UV image, in which the fluorescent silica beads are visible. Then those images were registered manually on the subsequently recorded scanning electron microscopy image. This required several steps, i. e. rotation, translation and shearing of the STED image. Basically the superposition was performed by using the calculated center of the fluorescent silica beads as markers and laying them on top of each other. In this case only one bead lay close enough to the histone structure to be covered in the STED image, but still the histone structure itself enabled the correct manual alignment of the two images. The shearing of the STED image, however, did not result from a 
deformation in the sample, but from a scanning issue of the setup, that was later solved.

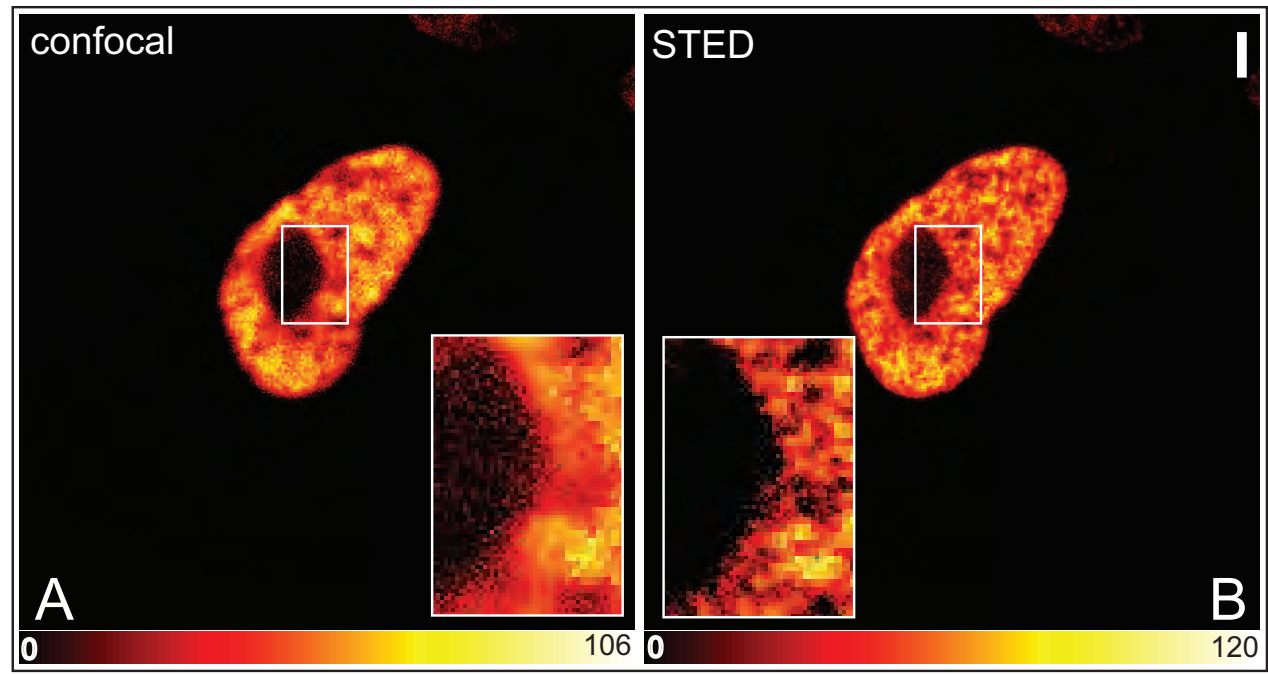

Figure 3.29: Confocal (A) and STED (B) images of his44 expressed in C. elegans after genetically encoding with Citrine, resin embedding and ultrathin sectioning of the animal. Section thickness: $100 \mathrm{~nm}$. Scale bar: $1 \mu \mathrm{m}$.

\section{Discussion}

The results presented in this Chapter can be seen as a fundamental progress for the targetorientated investigation of ever smaller biological structures. Genetically encoding with FPs is a crucial labeling method, not only for the research on the nematode C. elegans. Accessing them with a nanoscopic resolution in all three spatial directions is highly desirable if not essential for the precise localization of the encoded proteins. We presented an approach to reduce the focal volume by a factor of more than 500 compared to previous studies. On top, the correlation of the obtained images allows to see information obtained for single proteins in a larger structural context and with even higher resolution.

Although the list of proteins, which are interesting and suitable candidates for this method, goes even in the simple model organism C. elegans far beyond this work, a few are instanced here. As a follow up to the investigation of sng1 it could be suspenseful to further investigate the mechanisms of neuronal communication. A potential candidate for further studies is the synapse defective protein syd2. It is located to the dense projection of synapses, but where exactly is so far unknown. Beside the precise localization of the protein, more information can be obtained from correlative studies.

Even if a multicolor-color setup for the parallel imaging of two different fluorescent proteins at the same time has so far not been established, important findings about the relation of two specific substrates can be won. If each protein is expressed in a different animal, the according high resolution fluorescent images can independently correlated with EM pictures. Studying the relation between the protein with the peripheral ultrastructure indirectly allows to draw conclusions about the relation and, together with in 
vitro methods and genetic disruption experiments, the interaction of the proteins themselves.

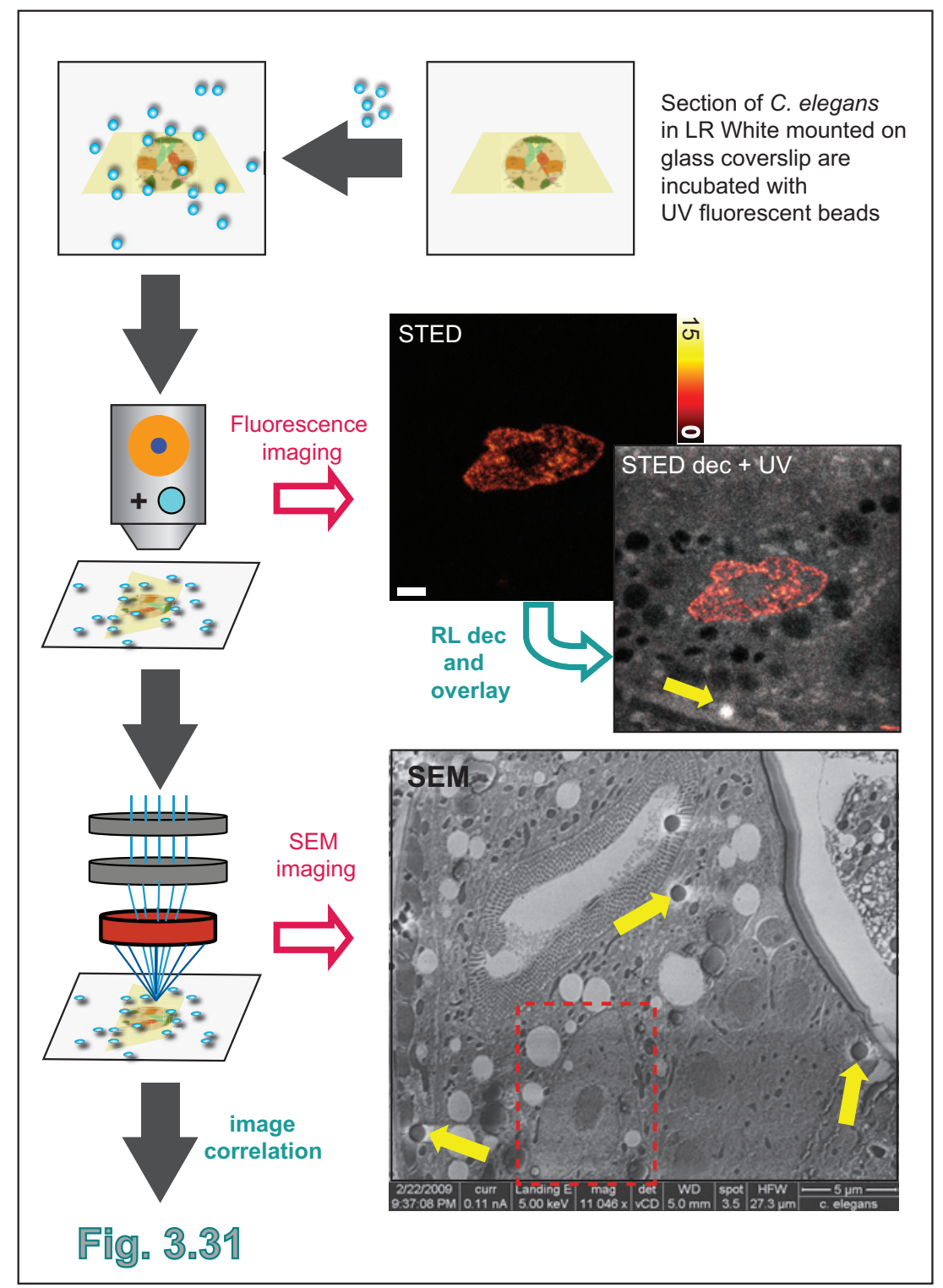

Figure 3.30: work flow towards correlative STED- and SEM-imaging. After incubation with fluorescent beads, the ultrathin sections are imaged with STED- and confocal (UV) microscopy and subsequently investigated with an SEM. After deconvolution of the STED image, it was overlaid the UV fluorescence image to localize the silica beads, which were used as alignment anchors. Beads are in STED and SEM data indicated by yellow arrows. Scale bar: $2 \mu \mathrm{m}$. 


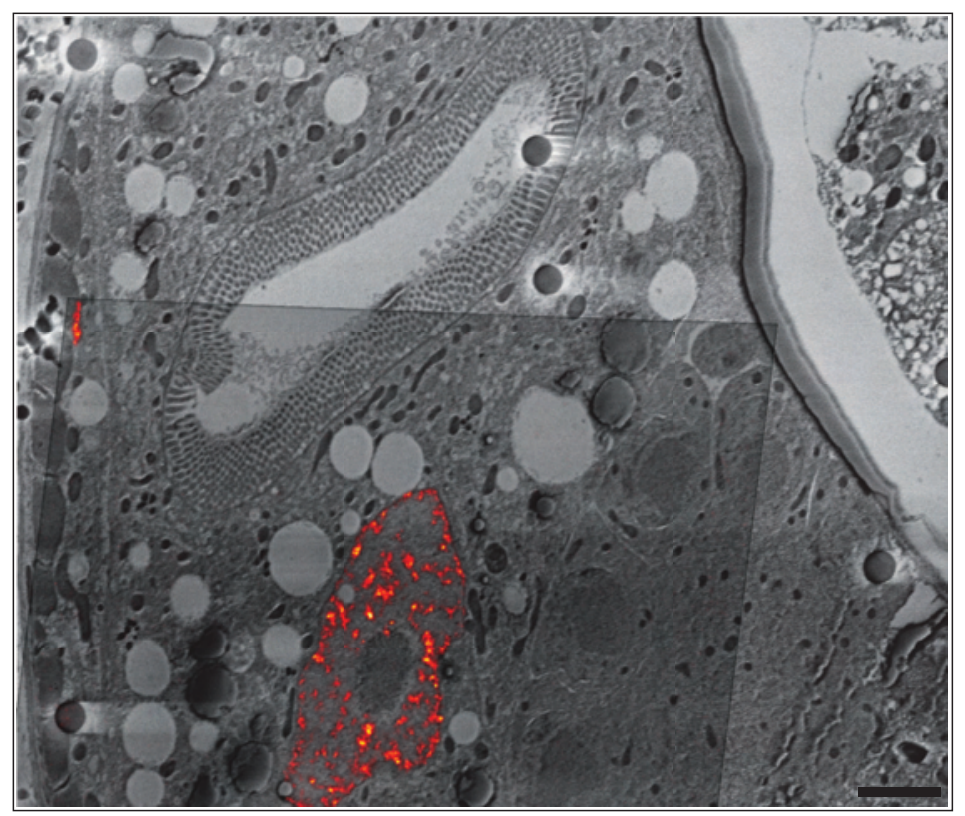

Figure 3.31: Correlative STED- and SEM-image of his44 genetically encoded with Citrine taken on an ultrathin section of $C$. elegans. Section thickness: $75 \mathrm{~nm}$. Scale bar: $2 \mu \mathrm{m}$.

In general, all fields of research grazed in this work, but also others, can benefit from the technique. C. elegans is an adequate and at the same time simple organism, which is easy to manipulate in any way, for numerous basic studies in life sciences. Thus, it is as interesting to promote neuroscience as well as mitochondrial research in the nematode as a model. However, the potential of the technique for sure goes even beyond this. Three-dimensional nanoscopic resolution in combination with genetically encoded labels is in this respect unambiguous. However, a lot of space for improvements of the method is left at this point.

First of all it remains to be evidenced, that preserving the fluorescence of FPs on ultrathin resin embedded sections while maintaining the ultrastructure of the sample is reproducible. This holds not only for further encoded proteins in other parts of $C$. elegans. That the results are transferable to other organisms has to be proven as well. Although the elaborated work flow was shown to be practicable for two proteins, further argument is necessary to develop it into a standard protocol. In any case, preparing even brighter signal would always go together with a higher optical resolution and remains a future goal.

With regard to electron-microscopic methods, transmission electron microscopy (TEM) definitely is superior to scanning electron microscopy (SEM). Preparing the samples directly on glass coverslips, which are because of their thickness and molecular density not a suitable substrate for TEM imaging, is to this end to be avoided. As a first 
experiment coverslips coated with a layer of pioloform were applied. Pioloform is a thin, electron-lucent but stable, film that can be removed from the glass together with the sections and can be transferred to a TEM grid. Establishing this method for correlative imaging would allow a further improvement in resolution.

Additionally, the presented technique can benefit from the imaging of several consecutive slices. Even if a whole animal is difficult to investigate for practical reasons, a reconstruction of a several $\mu \mathrm{m}$-thick tissue block would provide a lot of complementary information.

Concerning STED microscopy, it would be a major progress to have several and more (photo-) stable fluorescent proteins, which can simultaneously be imaged. Multicolor imaging would allow an even more detailed insight into the relation of two associated proteins and their correlated functions. 


\subsection{Application III: Nanoscopy on mammalian tissue}

As fruitful as the research on cultured cells or simple model organisms, like drosophila, C. elegans or zebrafishes is, as provocative is the investigation of the more complex mammalians. The house mouse mus musculus is the most common research organism in this field. Since the early 80s it is possible to grow so called transgenic and knockout mice, which are genetically manipulated [90, 172, 51]. This paved the way for the generation of fluorescent mice by genetically encoding GFP [88.

However, imaging a small animal or even it's fractional organs is a challenge. While already parts of a mouse brain, for instance, span a quite large volume of tens of cubic millimeters, the axonal and dendritic processes comprising a certain circuit are extremely fine and tortuously interconnected. Consequently, they call for a resolution in the range of nanometers.

In order to disentangle the neuronal network a resolution on the nanometer scale is indispensable. This is ambitious, not only because imaging a whole neuronal circuit with nanoscopic resolution would yield a tremendous amount of data (petabytes). To access a three dimensionally extended structure with a nanoscopic resolution, mechanical ultrathin sectioning is highly advantageous. How laborious this method is for threedimensionally extended specimens can be seen from the fact that even tracing the nervous system of C. elegans, which is less than $0.01 \mathrm{~mm}^{3}$ small, took a whole decade [178, 57]. Accordingly, it is over-ambitious to perform the same manual technique on mouse brains or even larger samples, what would go along with up to a million ultrathin slices.

Thus, Lichtman and coworkers, who are in fact working on the mapping of the mouse brain, came up with a new approach: the automated tape collecting in a lathe ultramicrotome (ATLUM) 61. By applying this technique, thousands of ultrathin sections can be cut and collected on a continuous plastic tape without the need of manual intervention. Hence, slicing at least parts of a mouse brain with a reliable quality of sections becomes possible.

Still, imaging thousands of brain tissue sections is not only a technical challenge in terms of slicing. Since new optical high-resolution approaches arose in the 1990s, several techniques compete with electron-microscopic methods on the nanoscale. All of them have their own drawbacks and advantages and are potential candidates for the imaging of brain slices. Which method is the most striking highly depends on the addressed problem in a particular case .

This Chapter demonstrates the general capability of STED nanoscopy to image fluorescently label brain tissue sections with high resolution. Moreover, the imaging on plastic tape instead of glass substrates is shown to be functional. To classify the method, stochastic GS-DIM imaging, another optical high-resolution technique, was also performed on those samples [38] $!^{5}$

\footnotetext{
${ }^{5}$ The in this Chapter presented work was performed as a collaboration with the lab of Jeff Lichtman at the Harvard university, in particular with Juan Carlos Tapia and Bobby Kasthuri. In detail, the transgenic mice were grown, embedded and ultrathin sectioned in Harvard. Mounting and imaging of the sections was subsequently done by me.
} 


\section{Methods}

The preparation of ultrathin slices of brain tissue is outlined in Fig. 3.32, The transgenic mice were prepared as described elsewhere 34]. After anesthetizing and heart-perfusing them, the desired tissue was dissected and embedded. The further processing is described in detail for each experiment.

For a first study, a multicolor animal expressing CFPS, YFPH and KOFP was used. The tissue was embedded in LR White and subsequently ultrathin sectioned $(50-70 \mathrm{~nm})$ by custom-built automated tape-collection in an ultramicrotome (ATUM). A photograph of the latter is depicted in Fig. 3.32. Initially, the ultrathin sections were collected on gelatin coated glass slides (about ten sections per slide) instead of plastic tape and sent to Göttingen.

Before immuno-staining the ultrathin sections with anti-GFP antibodies, the slides were briefly heated to about $90^{\circ} \mathrm{C}$ to prevent the gelatin from swelling. The immunofluorescence-labeling was achieved with secondary antibodies coupled to ATTO590. Subsequently, the sections were embedded in Mowiol.

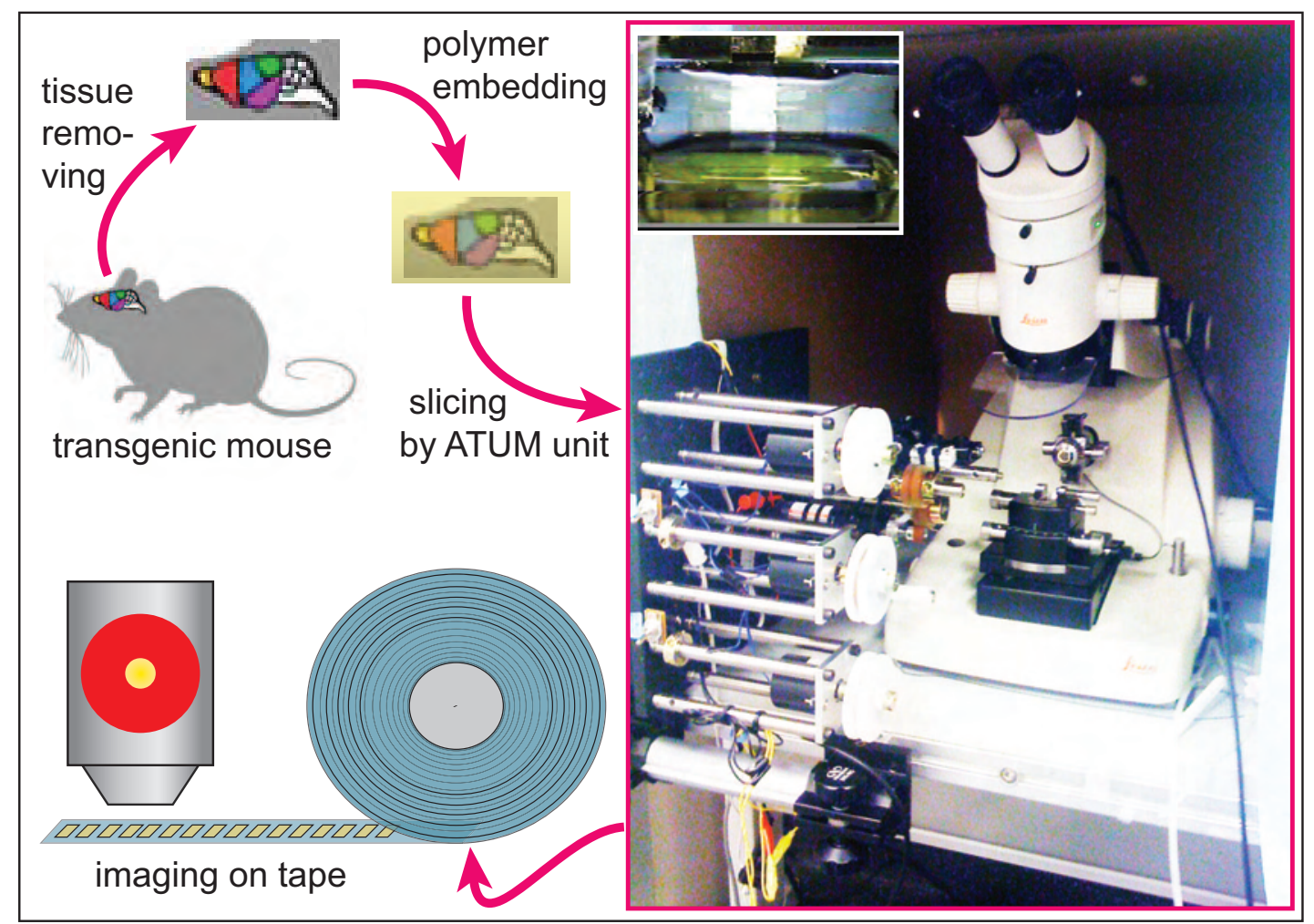

Figure 3.32: Sample preparation using the ATUM for high resolution imaging of ultrathin slices on plastic tape. The transgenic mice were anesthetized and heartperused before the desired tissue was dissected. After polymer embedding of the samples, they were cut ultrathin and collected on plastic tape in the ATUM unit for subsequent high-resolution imaging. 


\section{Experiments and results}

For STED imaging of the ultrathin sections the same setup, which had been described in Chapter 3.2 for the imaging of mitochondria was applied. The excitation and STED power in the aperture were about $0.5 \mu \mathrm{W}$ and $3.5 \mathrm{~mW}$ respectively. A pixel dwell time of $0.2 \mathrm{~ms}$ was used to acquire $20 \times 20 \mu \mathrm{m}$ images with a pixilation of $10 \mathrm{~nm}$. No subsequent mathematical deconvolution was carried out. All images shown are raw data. The confocal images were recorded with a pixel size of $40 \times 40 \mathrm{~nm}$. The pixel dwell time and the excitation power were equal to those applied for the STED image acquisition.

For GSDIM imaging the samples were prepared the same way, but immuno-stained with ATTO532. The setup, which was used for data acquisition was described elsewhere in detail [36, 16]. For fluorescence excitation a continuous-wave laser at $532 \mathrm{~nm}$ (Verdi V5, Coherent Inc, Santa Clara, CA, USA) was used. The imaging was performed with an oil immersion objective lens (HCX PL APO 100x/1.4 oil, Leica Microsystems, Wetzlar, Germany) generating a $12 \mu \mathrm{m}$ large excitation spot. The detection was realized in epi-direction on an EM-CCD camera (IXON-Plus DU-860, Andor Technology, Belfast, Nothern Ireland), using an image pixilation of $20 \mathrm{~nm}$. As described elsewhere, it has to be smaller than half the desired resolution 153. To obtain a high resolution image, about 85000 widefield frames were detected applying a dwell time of $5 \mathrm{~ms}$ for each image. Every 10000 images, the dye was reactivated by a $\mu$ s UV pulse delivered from a continuous-wave $375 \mathrm{~nm}$ laser (iPulse-375, Toptica Photonics AG, Gräfelfing, Germany). The calculation of the high resolution image from the previously up added diffraction limited data set was performed with custom-written MATLAB scripts (The Mathworks, Natick, CA) as described by Föllling et al [38].

For the collection of ultrathin slices on plastic tape, YFP16 mouse tissue was embedded in Epon. Those mice express YFP in all motoneurons [6]. The sectioning was carried out with the custom-built ATUM and collected on Lumox tape (Dupont, Wilmington, Delaware, USA). To amplify the fluorescent signal, the sections were after embedding and ultrathin sectioning immuno-stained with anti-GFP antibodies. After immuno-staining the tape was sent to Göttingen.

Upon arrival, the specimen were embedded in $98 \%$ TDE and subsequently imaged with the STED setup previously mentioned, except that only one STED beam was used. This resulted in a by a factor of two reduced STED beam power of $1.9 \mathrm{~mW}$ and thus a slightly poorer resolution. The acquisition time was elongated to $1 \mathrm{~ms}$ per $20 \mathrm{~nm}$ pixel for the dimmer samples. The imaging was performed directly through the backside of the tape, with no coverslip between the sample and the objective.

For the imaging of the synapses at the neuro-muscular junctions (NMJ) of a P0 muscle, specific pre-embedding immuno-staining for synaptophysin and for neurofilaments was carried out. The P0 muscle is localized in the neck of the animal and can thus easily be dissected. The further treatment of the tissue, including sectioning, tape collection, mounting and imaging was the same as for the YFP16 mice.

\section{Results}

At the beginning it was tested, whether STED microscopy would work on ultrathin brain tissue sections. The effect of the high-power STED beam on the dense tissue and the 


\subsection{Application III: Nanoscopy on mammalian tissue}

embedding polymer was not known, fluorescence labeling was a major concern. Although the mice were forced to express one or more FPs, the observed signal on ultrathin sections was not sufficient to perform STED imaging. Thus, enhancing post-embedding immunolabeling with organic dyes proved to be a viable option.

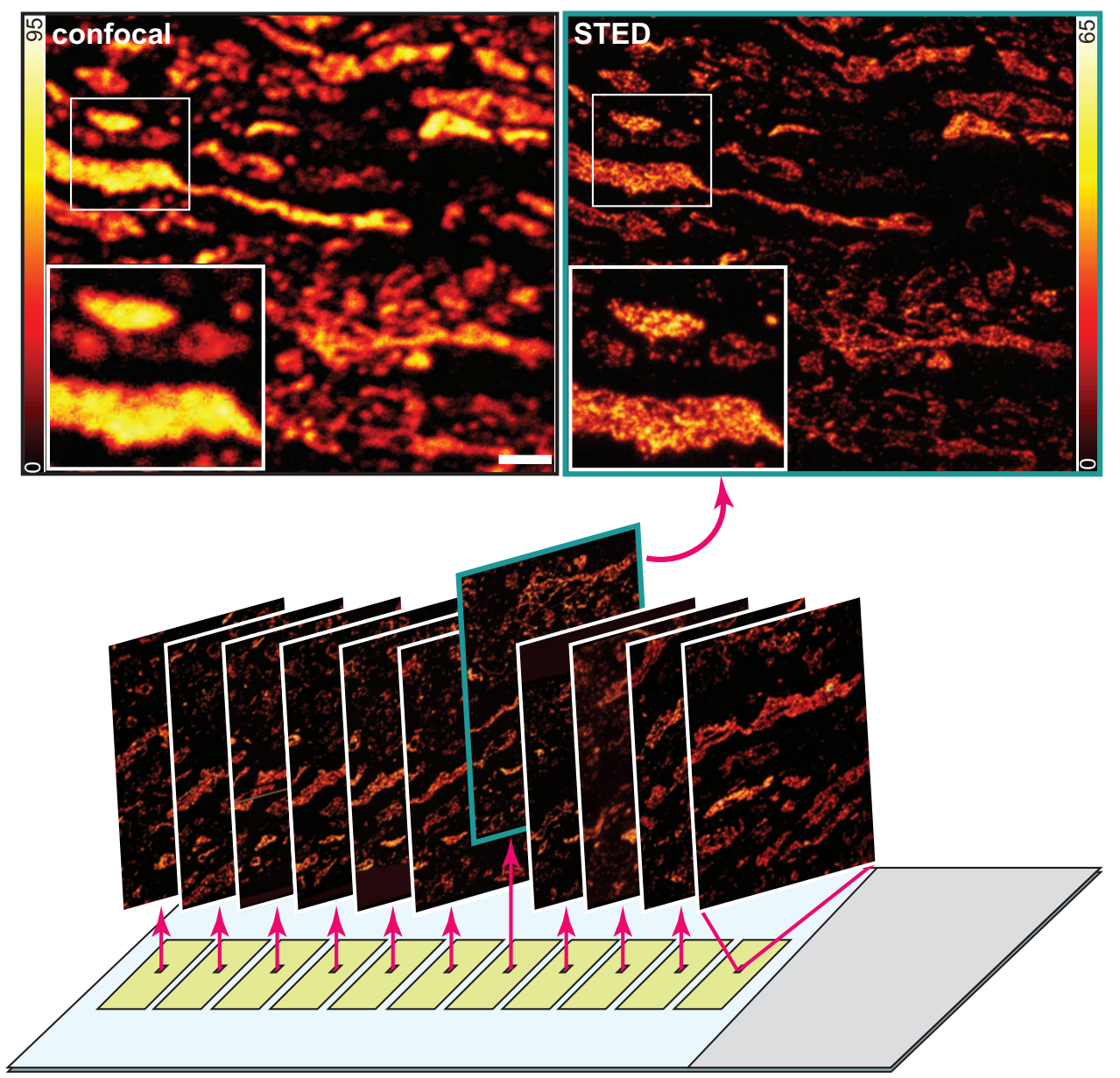

Figure 3.33: Stack of post-embedding immuno-stained ultrathin sections imaged with STED nanoscopy on gelatin coated coverslips. For one section the STED image (top right) together with the according confocal image (top left) is shown enlarged. The boxed area in the images is enhanced for both images. Section thickness: $50 \mathrm{~nm}$. Scale bar: $2 \mu \mathrm{m}$.

In the course of the experiment it was found that tissue sections tend much more to unspecific antibody reactions than does a layer of cultured cells. As a consequence, the background signal caused by staining artifacts is remarkably increased. The latter is especially misguiding for brain tissues sections, since the single spots are easily misinterpreted as cross-sectioned fine axons.

To increase the SNR an extraordinary bright structure was desirable for the first ex- 


\section{Experiments and results}

periments. To this end, a mouse simultaneously expressing three different FPs in it's motoneurons was chosen. The fluorescent labeling was enhanced by staining the final ultrathin sections with an anti-GFP antibody. Post-embedding labeling is more efficient, since the antibody reaction is not limited by the tissue penetration. In addition, an effect of the polymerization reaction on the fluorophore is excluded.

As the staining of the ultrathin sections goes along with several washing steps and changes of the added solutions, the employed microscope slides were in advance coated with gelatin. This made the sections adhesive on the glass.

The immuno-stained sections were mounted in Moviol and could successfully been imaged by STED microscopy. As shown in Fig. 3.33, a stack of 11 subsequent images, each of them $20 \times 20 \mu \mathrm{m}$ in size, was acquired. Due to the limited available scanning range only a very small part of a section, indicated by the small square on the yellow sections in Fig. 3.33 could be addressed in one scan.

To give an idea of the achieved resolution refinement, one of the images is shown enlarged, together with the according confocal image. The resolution enhancement is clearly obvious. Within the confocal imaging mode most of the structures appear quite blurred. In contrast, in the STED images, very tiny axonal substructures can be identified within a single nerve.

The high resolution images indicate that STED imaging might serve as a tool to follow single neuronal processes throughout a large stack of tissue. This was so far only possible by means of electron-microscopic methods. It is attractive to pursue this project with light microscopic techniques, since a single neuron, which expresses a FP can be tracked and easily identified within each section of the stack.

To test if comparable results can be obtained with stochastic methods, the same experiment in terms of the sample preparation was performed with GSDIM. Instead of ATTO590, ATTO532 was used for immuno-staining. ATTO532 is a standard dye for the application in GSDIM microscopy.

The detected high resolution image as well as the widefield image are shown in Fig. 3.34 . Again, the resolution enhancement is clearly obvious. However, while single bright spots had a FWHM of only $30 \mathrm{~nm}$ (see lineprofile in Fig. 3.34. C), the resolution on more densely labeled parts of the neuronal structure was a bit poorer than in the STED images (about $50 \mathrm{~nm}$, see lineprofile in Fig. 3.34, D). It is known, that high- resolution imaging with stochastic methods gets difficult as structures are more tightly labeled. Thus, the applied sample with its dense structures was not optimally adapted to the technique. In addition, a further modification of the acquisition mode might have provided better data. Still, GSDIM imaging was not further pursued for two reasons.

Firstly, since the images have an intrinsic spotty appearance. Hence, compared to STED microscopy, it is even harder to decide, which signals belong to the structure of interest and which are just due to artifacts. The latter might be caused by staining, but also, as observed in some samples, due to foldings and wrinkles within the ultrathin sections.

Secondly, the homogeneous background signal, which is normally present and limits the resolution significantly in stochastic high resolution methods. The latter is quite high on resin embedded sections, in particular, if auto-fluorescent polymers like epoxide resins 


\subsection{Application III: Nanoscopy on mammalian tissue}

are used (see Chapter 3.1). Additionally, as imaging the samples on plastic tape was the final goal, further destructive signal is likely to be caused by the collecting tape.

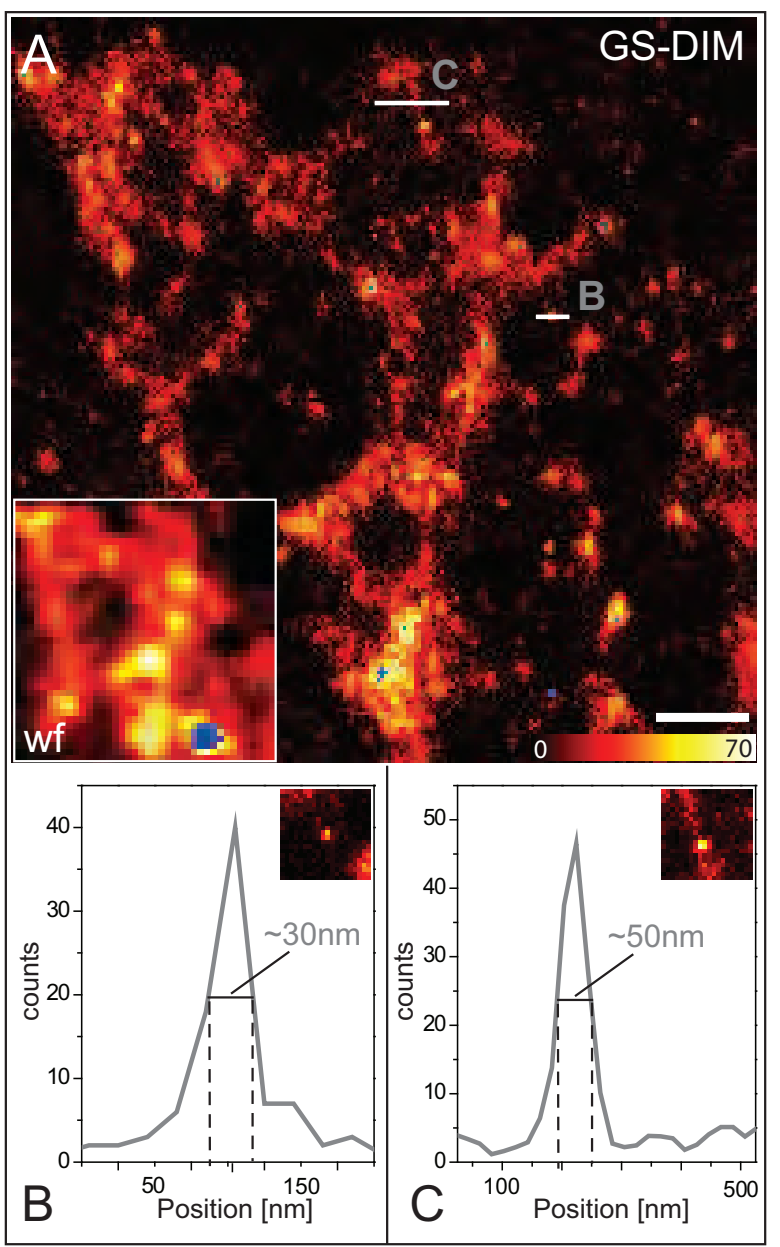

Figure 3.34: A: GSDIM and widefield (inset) image of a similar structure as in Fig. 3.33. Scale bar: $500 \mathrm{~nm}$. The lineprofiles indicated in A are shown in B (single spot) and $\mathrm{C}$ (continuous structure) respectively.

All further STED experiments were performed on slices collected on plastic tapes. This is indicated by the bluish highlighted background of the figures. Instead of Mowiol, the samples were mounted in TDE. In combination with plastic tape, Mowiol was observed to melt under the STED laser irradiation.

Sticking to the post-embedding labeling approach, a dimmer structure was chosen for the next experiment. To this end, the examined animal was genetically modified such that it expressed only one FP, YFP, in the cellular somata.

As the hydrophobic LR White sections were observed to be less adhesive on plastic tape and tend to detach and wrinkle, Epon was chosen as the embedding medium. In addition, the achieved section quality was enhanced and more reliable. The YFP labeling 


\section{Experiments and results}

of the ultrathin sections was amplified as previously described with an anti-GFP antibody coupled to ATTO590. This step was at this point even more vital, since after Epon embedding the signal from FP completely vanishes.

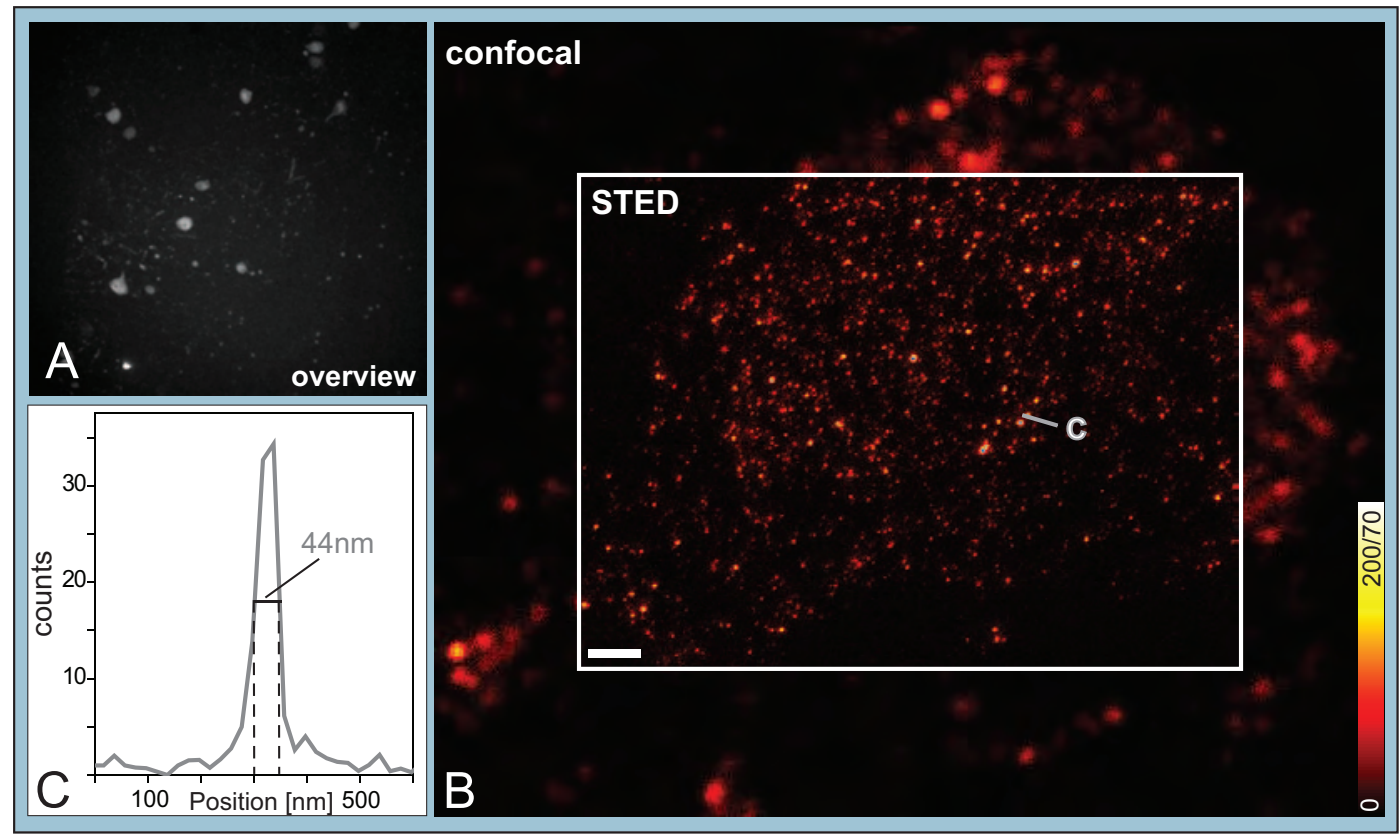

Figure 3.35: A: Widefield image (20x magnification) of ultrathin sectioned cellular somata in brain tissue, immuno-labeled for YFP. B: Confocal (periphery) and STED (center) image of the same image as in A. C: Lineprofile taken from the STED image at the indicated position. Section thickness: $75 \mathrm{~nm}$. Scale bar: $1 \mu \mathrm{m}$.

Several plastic tapes were tested for the collection of ultrathin slices and subsequent STED imaging, among them Mylar, Kapton, a polycarbonate tape and Lumox. Except Kapton, which strongly absorbed the irradiation from the STED beam, all of them were more or less suitable for optical high-resolution imaging. Finally Lumox tape was used, which had a thickness of only $25 \mathrm{~nm}$ and thus gave the lowest background signal.

The imaging was performed directly through the tape. This allowed to control the flatness of the tape after mounting. Even more important, bearing the investigation of a large stack of sections in mind, spatially constraints by the size of the coverslips are avoided, when meters of tape are to be imaged.

An overview image of sectioned cellular somata is shown in Fig. 3.35, A. Due to the restricted scanning range of the STED microscope, only one cell could be addressed within a high resolution image. A representable STED image is pictured in Fig. 3.35 , B. The periphery indicates the resolution achieved in the confocal imaging mode. The diffraction limited spots decompose in the STED image to several smaller ones, which are clearly separated. The size of the spots was reproducibly slightly below $50 \mathrm{~nm}$, pointed up 
by the lineprofile depicted in Fig. 3.35, C. This value of the FWHM equales the current resolution of the STED microscope.

Following the results of those initial studies, the challenging goal to image serial sections of a spatially extended specific structures was addressed.

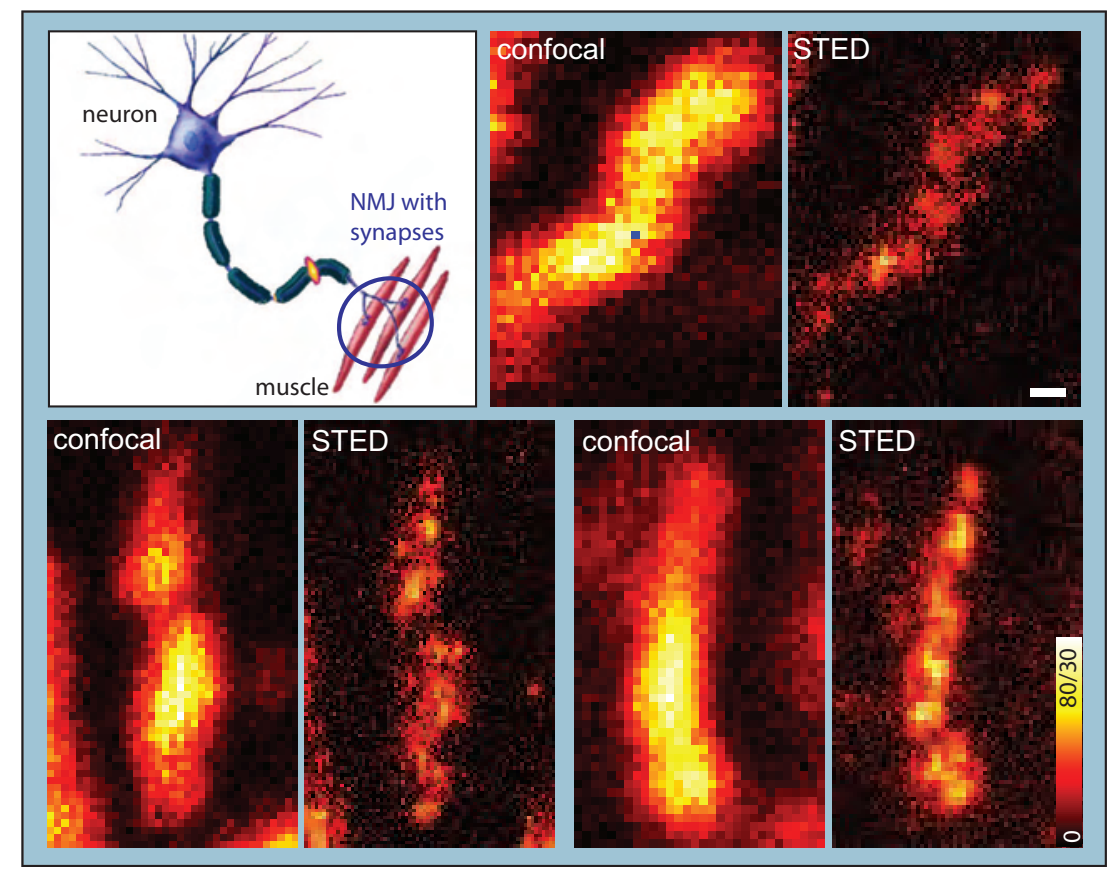

Figure 3.36: Confocal and STED images of synapses at the neuro-muscular junction of the P0 muscle, after embedding and ultrathin sectioning immuno-stained with ATTO590 against SNAP-25. A sketch of the cellular context is also shown (bottom right). Section thickness: $75 \mathrm{~nm}$. Scale bar: $200 \mathrm{~nm}$.

Hence, the a NMJ, which is smaller than a brain circuit, was assumed to be a wellsuited test system. A NMJ of a very young animal is about $5 \mu \mathrm{m}$ in size. Axons run together in several regions of a nerve, but then each one gives rise to synapses, comparable to a trunk ending up in many little branches (see sketch in Fig. 3.36). Each muscle fiber is connected to one of those synapses. So far it is unknown, how many axons end up in a single of those synapses. Counting the total number of axonal inputs into a single NMJ is therefore an interesting and challenging project, which gets accessible by high resolution light microscopy. In addition, it would be interesting to investigate, if the number of axons giving inputs to a single synapse changes when an animal grows older. Electron-microscopy is not applicable in this context, since a specific effective labeling method is lacking.

However, performing post-embedding staining on a large number of ultrathin sections is quite laborious. Additionally, a large volume of antibody solution would be required. Therefore, it is much more straight-forward to stain the whole tissue block before embedding it into plastic. For the previously mentioned reasons, the fluorescent signal is 


\section{Experiments and results}

significantly lower in such case. Furthermore, staining artifacts become more likely when the antibodies have to penetrate a large tissue volume.

To specifically label the synaptic sites of a NMJ, synaptophysin was immuno-stained. It is a prominent marker for synaptic vesicles and thus in this experiment instrumental. In addition, neurofilaments were stained at the same time with the same color to facilitate the orientation within the tissue block.

Three STED images of different synapses, immuno-stained before embedding, are shown in Fig. 3.36. As the structures are not as bright as before (roughly a third of the signal), the final resolution is less impressive. Thus, counting single axonal inputs is hardly possible without additional image deconvolution. However, in comparison with the confocal images, the substructure is much better resolved in the STED data.

An improving of the staining is definitely necessary to address the previously posed question. Still, STED nanoscopy features the premise for further experiments in this respect. Not only a single synapse will be reconstructable by imaging brightly labeled ultrathin sections on tape. As a long-term objective, also the whole endplate of a NMJ, comprising 100 - 1000 single synapses, might be accessed.

\section{Discussion}

The presented results encourage more ambitious projects. Even the small site of a NMJ opens up a number of interesting questions, which call for nanoscopic imaging techniques. For some reasons optical methods might be favored in this respect.

However, talking about STED nanoscopy in particular, the next outstanding jobs are obvious. One task is certainly to enable a larger region of interest to be addressed within a single image scan. Ideally the scanning range would come up to the size of a whole tissue section. This would dramatically reduce the reconstruction efforts for lager tissue blocks.

Another issue is the performance of multi-color imaging (three or more colors) and the imaging of FPs. However, especially, as the imaging of FPs themselves is problematic in STED nanoscopy, this is inevitably accompanied with further advancement of the immuno-staining protocols.

Even more challenging than the comparably small NMJ is the imaging of the connectome. The connectome is a map of the full set of neurons and synapses within the nervous system of an organism. The gold standard methods to reach the connectome are tract tracing and also electron-microscopy, but both suffer from their own drawbacks.

Some progress has recently been made, when Lichtman and coworkers published their brainbow approach [116, 113. It allows to individually map a single neuron marked by its unambiguous color over a long distance. Still, the entire wiring of single neurons is an untouched land. While confocal methods do not allow a nanoscopic insight into the latter, electron-microscopy suffers from the lack of specific markers for an individual neuron. This makes the tracking of axonal processes over a long distance highly complex. STED nanoscopy might have a key function in this respect. Identifying a single neuron over large distances is possible by the omni-presence of GFP in its interior. Together with the provided resolution a detailed study even of very tiny processes is a realistic 
aim.

On top, a correlative study with EM of the same tissue would allow to identify synapses along the neuron and thus comprise to the construction of the whole connectome. However, in other experiments, alternative nanoscopy methods might be superior to STED. Although the data acquisition is faster compared to other techniques, in particular electron-microscopy, STED nanoscopy is substantially limited concerning the direct imaging of FPs, multicolor-imaging, and the scanning range addressable within a single image.

All these problems might be less severe in stochastic widefield techniques like PALM or STORM. Still, they also do not offer a state-of-the-art solution. Especially if specific immuno-staining is demanded, a thorough labeling gets even more critical in those methods. In addition, the fluorescence background of either the collecting plastic tape or the embedding media will restrict those methods to very bright structures.

But for all that, the highest possible spatial resolution is provided by electron-microscopy. Even for SEM, which is faster and more straight-forward than TEM, a reproducible resolution of $5 \mathrm{~nm}$ is the standard. Thus, it will always be a nice complementary tool in correlative studies. While the specific staining of structures might be less straight-forward, the embedding of fluorescent structures into their peripheral context is unmatched by any other method.

To summarize, each available nanoscopic method will certainly have its place in one or the other application. But all of them have one challenge in common: Imaging a whole mouse brain takes a tremendous amount of imaging time, no matter whether it is done by electron- or light-microscopic methods. And even more, the resulting data volume is just terrific. Therefore, one is well advised to balance out between a nanoscopic resolution and the sample volume. For the studying of processes at a single synapse or a single neuron nanoscopic resolution might be truly valuable. For a whole brain, in particular of larger animals as mice, it is at the moment utopistic. 



\section{Conclusion and Outlook}

In this thesis, ultrathin sections of biological tissue were successfully investigated by Stimulated Emission Depletion (STED) nanoscopy. Due to scattering and aberrational effects, thick biological structures are difficult to image with visible light, particularly at the highest resolution. Here, these problems were effectively addressed by a novel technique resting on ultrathin sectioning of the sample to a thickness well below the wavelength of light (typically $50-100 \mathrm{~nm}$ ).

Due to three main reasons, the usual problems occurring during the investigations of thick samples are avoided with this technique. First, the explored sample volume within the (confocal) focus is significantly reduced through ultrathin sectioning. As a result, fluorescence from off-focal planes is absent, giving rise to a markedly increased imaging contrast. Second, dense structures are clearly revealed with subdiffractional axial resolution at distances below the size of a confocal spot. At the same time, this also increases the probability to detect dim features of the sample. Third, each ultrathin layer of the specimen is investigated independently, such that destructive photo-bleaching in the periphery of the focal plane is avoided enabling a high resolution study throughout the whole labeled structure.

Ultrathin slices require embedding of the samples into polymers. As a first step, this study involved a range of tests addressing the suitability of three commercially available embedding media, i.e. epoxide, acrylate and melamine resins. Specifically, their refractive index and their auto-fluorescence at various excitations wavelengths were tested. Furthermore it was investigated, if the spectroscopic properties (absorption or emission spectra, fluorescence lifetime, quantum yield) of selected fluorophores are altered upon embedding

Based on these initial studies, the concept of fluorescence inhibition by STED was validated on biological, ultrathin sectioned samples and applied to the imaging of various proteins. An over-all subdiffractional resolution was achieved on spotty structures like nuclear speckles, histones or clustered synaptic proteins, as well as on cellular filaments such as tubulin and vimentin. A lateral resolution below $30 \mathrm{~nm}$ was also observed on $50 \mathrm{~nm}$ thin sections of the tightly packed inner-mitochondrial membrane.

A single-cell layer of cultured neurons was analyzed in more detail. It was shown that ultrathin sectioning in combination with STED allows one to reveal the exact position of synaptic proteins within a dead synapse. It was also shown that ultrathin sections are easy to combine with two-color STED imaging, facilitating colocalization studies on different synaptic proteins. Importantly, high-resolution images of adjacent sections were successfully rendered yielding a three-dimensional two-color image of the protein distribution in an axon.

In order to address a more complex organism, proteins of the nematode C. elegans were 


\section{Conclusion and Outlook}

investigated. As suitable antibodies are rare for this organism, the structures of interest were genetically encoded with fluorescent proteins. In contrast to organic fluorophores, fluorescent proteins are highly sensitive to fixation and dehydration, which normally give rise to a substantial loss of the proteinogenic fluorescence. However, sustaining the signal is important especially for STED nanoscopy, where fluorescent proteins may suffer from high power irradiation and accompanied photo-bleaching. To still allow the use of fluorescent proteins, the common embedding protocol had to be adapted. High pressure freezing in combination with soft fixation agents $\left(\mathrm{KMnO}_{4}\right)$ and a controlled $\mathrm{pH}(\sim 7)$ were employed to allow the imaging of the samples with STED with a lateral resolution of about $50 \mathrm{~nm}$. The explored protocol allows for the specific nanoscopic investigation of any protein of the model organism C. elegans that can be genetically encoded with fluorescent markers. To properly localize the measured structure within the worm, we aligned the resulting image with electron-microscopic data of the identical ultrathin section.

Motivated by the long-term objective to investigate large samples with nanoscopic resolution, STED nanoscopy was also successfully performed on ultrathin sections of mammalian tissue. Imaging of the immuno-stained mouse tissue resulted in a high lateral resolution below $30 \mathrm{~nm}$. Bright samples were also subject to a stochastic technique called GSDIM imaging. We showed that these data do in fact compare with STED images, and thus hold the future promise of GSDIM becoming a complementary technique to STED. A further step into establishing a technique for probing large specimens was taken by integrating the Automated Tape-collection UltraMicrotome (ATUM) into the work flow. This substantially facilitates the reproducible collection of a large number of serial slices by collecting them on a continuous plastic tape. STED was successfully performed on ultrathin sections collected on plastic tape, illustrating the compatibility of light microscopic high resolution techniques with these automatically prepared samples. Importantly, this method avoids the laborious manual sectioning procedure, and thus allows arbitrarily thick samples to be examined at nanoscopic resolution. However, the fluorescence labeling of spatially extended tissue blocks calls for further improvement.

To conclude, the field of optical far-field imaging was dramatically stimulated by the upcoming of several methods to undercut the diffraction limit of resolution. Currently, their further refinement is mainly limited by the size, the brightness and the photostability of the applied fluorescent labels. Their success is supported by fast progress in the computational handling of large data volumes and the increasing variety of tools for sample preparation, particularly in the field of specific labeling. Based on this, the examination of more and more complex organisms with nanoscopic resolution might be enabled. This includes the investigation of ever larger and thicker samples - the limits remain to be explored.

However, the weak point of all optical high-resolution methods remains the threedimensional investigation of arbitrarily thick samples. This holds for STED or RESOLFT techniques, as well for stochastic methods like PALM and STORM. Some sophisticated approaches tackle this difficulty and provide a high three-dimensional resolution on semithick samples (e. g. isoSTED or iPALM), but at the cost of a complex setup and/or data processing, respectively. Hence, mechanical ultrathin slicing is an interesting complement for all optical high-resolution methods. At the outset, due to sample-thicknesses of only 
a few tens of nanometers, this method enables a nanoscopic axial resolution even for straight-forward high-resolution techniques.

Stochastic widefield techniques can benefit even more from the mechanically introduced reduction of the observed sample volume. In the most basic imaging modes, a precise axial localization of the detected signals is not possible. In addition, a dense labeling of the sample decreases the imaging contrast and impairs the lateral resolution significantly. Thus, mechanical ultrathin sectioning can substantially refine both the axial and the lateral resolution of stochastic high resolution techniques. This is possiple inspite of the fact that the imaging contrast might be lowered by a perturbing auto-fluorescence of the embedding medium. Beyond that, in some applications, stochastic imaging might feature certain advantages over STED imaging. First, a larger region of interest can be imaged at once, second, the imaging of fluorescent proteins is more compatible with stochastic methods and third, multi-color imaging can be performed with less complex setups.

The present work established that STED imaging on mechanically prepared ultrathin sections provides a powerful tool to address numerous questions of life science. To this end, five valuable techniques, which previously proved indispensable for the study of biological structures, were successfully employed to complement the method, i.e. twocolor imaging, three-dimensional image reconstruction, the investigation of fluorescent proteins, correlative studies by electron-microscopy and the imaging of serial sections on continuous plastic tape. Beyond that, ultrathin sectioning also holds the promise of significantly improving stochastic high-resolution far-field methods. 



\section{Appendix}

\section{Polymerization of embedding media for screening experiments (Section 3.1)}

Nanoplast The embedding kit was bought from Polysciences, Inc. (Warrington, PA) For the reaction mixture $1 \mathrm{~g}$ of MME7002 (hexamethylolmelamine-methyl ether, $70 \%$ solution in water) were stirred with $0.025 \mathrm{~g}$ of B52 ( $p$-toluene sulfonic acid) until a clear solution with a $\mathrm{pH}$ of about 4 was formed. The mixture was either directly added onto a glas coverslip or onto cells in an aqueos buffer using a BEEM mold (Plano GmbH, Wetzlar, Germany) for shaping the block. The sample was stored in a desicator without vacuum over silica gel at RT for $24 \mathrm{~h}$ and afterwards at $40^{\circ} \mathrm{C}$ for another $24 \mathrm{~h}$ to ensure infiltration and drying of the polymer. Subsequently it was removed from the desicator and put at $60^{\circ} \mathrm{C}$ for another $48 \mathrm{~h}$ for polymerization.

As the embedding kit was not commercially available anymore, the reaction mixture was formed by solving $p$-toluene sulfonic acid $(0.025 \mathrm{~g}$, Sigma-Aldrich GmbH, Taufkirchen, Germany) in distilled water (0.295 g) and adding HMME (hexamethylolmelamine-methyl ether $0.69 \mathrm{~g}$, , TCS Europe) afterwards.

Araldite 6005 Araldite6005 was bought from SPI supplies/Structure Probe, Inc., West Chester, PA. For the reaction mixture $1 \mathrm{ml}$ Araldite 6005 (resin) was mixed with $1.0 \mathrm{ml}$ DDSA, $0.5 \mathrm{ml}$ BDMA and $0.3 \mathrm{ml}$ DBP. The mixture was cured at $80^{\circ} \mathrm{C}$ for $12 \mathrm{~h}$.

Durcupan The embedding kit was ordered from Fluka AG, Buchs, Schweiz. $0.5 \mathrm{ml}$ of component A were shaked with 1 of component B. Afterwards 1 of component $\mathrm{C}$ were added yielding abrown solution. Finally $0.02 \mathrm{ml}$ of component $\mathrm{D}$ were added. Curing was performed by storing the sample at $45^{\circ} \mathrm{C}$ for $1 \mathrm{~d}$ and $60^{\circ} \mathrm{C}$ for another $4 \mathrm{~d}$.

Epon812 The components were ordered at Serva Electrophoresis GmbH, Heidelberg, Germany.5.0 g of Epon812 (glycid ether 100) were mixed with $5.5 \mathrm{~g}$ of DDSA, $1.25 \mathrm{~g}$ NMA and $0.3 \mathrm{~g}$ BDMA, while avoiding air bubbles to form. Curing was perforemd at $60^{\circ} \mathrm{C}$ for $4 \mathrm{~d}$.

Poly/Bed 812 The reaction kit was bought from Polysciences Ing. Warrington, PA, USA. $5 \mathrm{ml}$ of Poly/Bed 812 were mixed with $3 \mathrm{ml}$ DDSA, $1 \mathrm{ml}$ NMA and $1 \mathrm{ml}$ DMP-30 unter $N_{2}$-atmosphere. The polymerization was performed at $60^{\circ} \mathrm{C}$ for $24 \mathrm{~h}$. 


\section{Appendix}

Quetol 651 The reaction kit was bought from Polysciences Ing. Warrington, PA, USA. $1.65 \mathrm{ml}$ Quetol 651 (ethylene glycol diglycidyl ether) were added to $3.35 \mathrm{ml}$ of NSA and after addition of $0.1 \mathrm{ml}$ DMP-30 and mixing polymerized at $60^{\circ} \mathrm{C}$ for $24 \mathrm{~h}$.

Spurr Low Viscosity The reaction kit was bought from Polysciences Ing. Warrington, PA, USA. $2.75 \mathrm{ml}$ NSA were mixed with $1.0 \mathrm{ml} \mathrm{VCD,} 0.1 \mathrm{ml}$ DER736 and $0.25 \mathrm{ml}$ DMA. The reaction mixture was cured at $70^{\circ} \mathrm{C}$ for $24 \mathrm{~h}$.

Lowicryl K4M The Lowicryl kit was bought from Polysciences Ing. Warrington, PA, USA. $0.27 \mathrm{~g}$ of crosslinker A, $1.73 \mathrm{~g}$ of monomer B and $0.01 \mathrm{~g}$ DBP were mixed by bubbling $\mathrm{N}_{2}$ through the reaction mixture to avoid the solution of oxygen during stirring. The samples were polymerized at $60^{\circ} \mathrm{C}$ within $4 \mathrm{~d}$.

Lowicryl K11M Using the same reaction kit as for Lowicryl K4M 9.5 g monomer I, $0.5 \mathrm{~g}$ crosslinker $\mathrm{H}$ and $0.05 \mathrm{~g}$ initiator $\mathrm{C}$ were mixed by bubbling $N_{2}$ through the reaction mixture. Polymerization wad induced by UV light within $24 \mathrm{~h}$.

Lowicryl H20M Using the same reaction kit as for Lowicryl K4M $1.12 \mathrm{~g}$ of crosslinker $\mathrm{D}, 6.38 \mathrm{~g}$ of monomer $\mathrm{E}$ and $0.05 \mathrm{~g}$ DBP were mixed by bubbling $N_{2}$ through the reaction mixture. The samples were polymerized at $60^{\circ} \mathrm{C}$ within $4 \mathrm{~d}$.

LR White The LR White embedding kit was purchased from Fluka AG, Buchs, Schweiz. The prepolymerization was done by adding $9.9 \mathrm{~g}$ catalyst to a $500 \mathrm{ml}$ bottle of LR White resin and thoroughly shaking. This mixture was stored at RT and upon usage directly polymerized within $24 \mathrm{~h}$ at $60^{\circ} \mathrm{C}$.

JB4 The reaction kit was bought from Polysciences Ing. Warrington, PA, USA. $0.083 \mathrm{~g}$ of the catalyst were completely solved in $6.7 \mathrm{ml}$ of solution A (monomer) under $\mathrm{N}_{2}$ atmosphere. Subsequently $0.27 \mathrm{ml}$ of solution B (initiaor) were added. The polymerization took place under cooling on ice and exclusion of oxygen within $4 \mathrm{~h}$.

Unicryl Unicryl was bought from Science Services, London, UK and could directly be used for polymerization. The curing was performed at $50^{\circ} \mathrm{C}$ within $2 \mathrm{~d}$.

Microbed Microbed was purchased from Electron Microscopy Sciences, Hatfield, PA, USA and could directly be polymerized at $50^{\circ} \mathrm{C}$ within $2 \mathrm{~d}$.

\section{Immuno-staining of samples (Section 3.2)}

PTtK2 (kangaroo rat) or Hela cells cells were grown on cover slips. Cells were fixed with $4 \%$ PFA (Sigma-Aldrich GmbH, Taufkirchen, Germany) in PBS (pH 7.4) for 10 min at $37^{\circ} \mathrm{C}$ and blocked with $2 \%$ BSA (Sigma-Aldrich GmbH, Taufkirchen, Germany) in PBS. 
Subsequently, cells were for $2 \mathrm{~h}$ at $22^{\circ} \mathrm{C}$ incubated with monoclonal antibodies diluted in PBS against several proteins:

mouse-anti-vimentin (Sigma-Aldrich GmbH, Taufkirchen, Germany);

rabbit-anti- $\beta$-tubulin (Sigma-Aldrich GmbH, Taufkirchen, Germany);

rabbit-anti-H3S10pH (Epitomics Inc., Burlingame, CA, USA);

rabbit-anti-giantin (abcam, Cambridge, MA, USA);

mouse-anti-SNAP-25 (71.1, Synaptic Systems, Göttingen, Germany);

mouse-anti-Sc35 (BD Pharmingen, San Diego, CA);

rabbit-anti-synaptophysin 92

After several washing steps, the primary antibodies were detected with secondary antibodies (Dianova GmbH, Hamburg, Germany) labeled with various ATTO-dyes (ATTOTEC GmbH, Siegen, Germany). To this end, the secondary antibodies were diluted in PBS and apllied for $2 \mathrm{~h}$ at $22^{\circ} \mathrm{C}$, followed by washing with PBS and fixation with $4 \%$ PFA in PBS for $5 \mathrm{~min}$. In case of Giantin the primary antibody was detected by a Fab-fragment (Dianova GmbH, Hamburg, Germany).

\section{Ultrathin sectioning (Section 3.2-3.4)}

The polymerized blocks were detached from the coverslips by dipping them into liquid nitrogen and cut with an ultramicrotome (EM UC6, Leica Microsystems GmbH, Wetzlar, Germany) using an ultrasonic diamond knife (Diatome AG, Biel, Switzerland). The slices were directly transferred onto glass coverslips.

\section{Preparation of Mowiol and section embedding (Section 3.2-3.3)}

25 g Polyvinylalcohol (Polysciences, Inc., Warrington, PA, USA), $88 \%$ hydrolyzed, MW 25.000, and $0.12 \mathrm{~g}$ DABCO (Sigma-Aldrich GmbH, Taufkirchen, Germany) were under slight heating solved in $100 \mathrm{ml}$ distilled, steril water. The mixture was stored in small amounts at $-20^{\circ} \mathrm{C}$. For embedding of sections on glass coverslips, an additional plasmacleaned glass coverslip was spin-coated with about $50 \mu \mathrm{l}$ of the prepared solution for $20 \mathrm{~s}$ at $5000 \mathrm{rpm}$ and added on top of the section before drying of the Mowiol solution. Imaging was performed after drying of the Mowiol.

\section{Immuno-staining of mitochondria (Section 3.2)}

PTtK2 (kangaroo rat) cells were grown on cover slips. Cells were fixed with $8 \%$ PFA (Sigma-Aldrich GmbH, Taufkirchen, Germany) in PBS (pH 7.4) for $10 \mathrm{~min}$ at $37^{\circ} \mathrm{C}$, extracted with $0.1 \%$ SDS (Sigma-Aldrich GmbH, Taufkirchen, Germany) in PBS, and blocked with $5 \%$ BSA (Sigma-Aldrich GmbH, Taufkirchen, Germany) in PBS. Subsequently, cells were incubated with a monoclonal mouse antiserum directed against the alpha subunit of the mitochondrial $\mathrm{F}_{1} \mathrm{~F}_{0}$ ATPase (Molecular Probes, Eugene, OR, USA). 


\section{Appendix}

The primary antibodies were detected with secondary antibodies (sheep anti-mouse; Jackson ImmunoResearch Laboratories, West Grove, PA, USA) custom labelled with the fluorophore KK114[171]. For isoSTED imaging, the PBS buffer was exchanged by a dilution series with TDE in PBS, finally resulting in an embedding medium of $97 \%$ TDE (Fluka AG, Buchs, Schweiz) in PBS. For ultrathin sectioning the cells were embedded in Nanoplast. The sections were taken up on glass coverslips and embedded in an medium of $97 \%$ TDE in PBS without application of a dilution series.

\section{Immuno-staining of neuronal hippocampal cells (Section 3.3)}

Rat hippocampal neurons were grown onto astrocytic monolayers. If necessary to facilitate the registration of neighboring xy-images within a z-stack, the cells were incubated overnight with a diluted suspension of two-color fluorescent silica beads at $37^{\circ} \mathrm{C}$. After fixation with PFA and permeabilization, achieved using $0.1 \%$ Triton X-100 for $10 \mathrm{~min}$ (both Sigma-Aldrich GmbH, Taufkirchen, Germany) the following antibodies were used diluted in PBS for immuno-staining $\left(5 \mathrm{~min}\right.$ at $\left.37^{\circ} \mathrm{C}\right)$ : anti-synaptophysin [92, antiSNAP-25 (71.1, Synaptic Systems, Göttingen, Germany), anti-syntaxin1 (HPC-1[7]), and anti- $\beta$-tubulin (Sigma-Aldrich $\mathrm{GmbH}$, Taufkirchen, Germany). After several washing steps with PBS secondary antibodies (Dianova GmbH, Hamburg, Germany) labeled with ATTO532 (ATTO-TEC GmbH, Siegen, Germany), ATTO647N or ATTO633 fluorescent dyes were apllied for $2 \mathrm{~h}$ at $22^{\circ} \mathrm{C}$, followed by washing with PBS and fixation with PFA in PBS. Finally cells were embedded in Nanoplast. Ultrathin sections were taken up on glass coverslips and embedded in Mowiol.

\section{Staining of fluorescent silica beads (Section 3.3)}

For staining the NHS-functionalized fluorescent silica beads (sicastar1-greenF, $300 \mathrm{~nm}$, $\mathrm{NH}_{2}$ - functionalized, $50 \mathrm{mg} / \mathrm{ml}$, micromod GmbH, Rostock, Germany) $70 \mu \mathrm{g}$ of ATTO633NHS-ester (ATTO-TEC GmbH, Siegen, Germany) were solved in $10 \mu \mathrm{l}$ of dry DMSO. Afterwards $500 \mu \mathrm{l}$ of the bead solution were added, ensuring the $\mathrm{pH}$ to be between 8 and 9 . The mixture was stirred at room temperature for $1 \mathrm{~h}$. The beads were seperated from the solution by centrifugation $(5000 \mathrm{rpm})$ and several times washed with Milipore water.

\section{Matlab script for the analysis of the protein cluster localization at synaptic vesicle sites (Section 3.3)}

function do_analysis(spamdir, varargin)

Do the analysis on all files in the current directory using the corresponding cluster positions from the 'spamdir' given. The script counts up from 00 to 99 and looks for files named $\mathrm{xx}-\mathrm{vis}-\ldots$ and $\mathrm{xx}-\mathrm{ir}-\ldots$ and a corresponding $\mathrm{xx}-\ldots$. spam file in the subdirection. It then does some thresholding operations on the 'ir' file, obtaining a mask that is supposed to mark the vincinity of release sites derived from areas with a high concentration of 
vesicles (synaptophysin). Next, it simply counts all clusters found in the 'vis' file manually and saved in the 'position' files that are within the mask and those outside the mask. The results for all files found are accumulated. It also calculates the average brightness of the clusters inside vs. outside the mask by integrating the intensity within a small rectangle around the cluster center.

params.debug $=$ false;

$\%$ radius of the disk within which the intensity of a cluster is

$\%$ determined. params.r_c $=2 ; \%$ radius of the erosion disk

params.r_e $=5$;

$\%$ radius of the dilation disk

params.r_d $=13 ; \%$ we threshold at this percentage of the 90

$\%$ median params.bg_thresh $=40$;

$\%$ subtract background ? which radius params.r_bg $=0$;

params $=$ spam_read_params(params, varargin);

strel_erode $=$ strel('disk', params.r_e, 0); strel_dilate $=$ strel('disk', params.r_d, 0);

strel_clust $=$ strel('disk', params.r_c, 0);

nhood_clust $=\operatorname{getnhood}($ strel_clust $) ;$ total_in $=0$;

sq_in $=0$; total_out $=0$;

sq_out $=0$; counts_in $=0$; counts_out $=0$;

for $\mathrm{i}=0: 99 \mathrm{~s} \_\mathrm{fn}=\operatorname{sprintf}\left({ }^{\prime} \% 02 \mathrm{~d}, \overline{\mathrm{i}}\right)$;

try

$\%$ read the first stack with $>=2$ dimensions img_ir $=$ omas_bf_read([ s_fn '-ir*' $]$,

-2 , 'double');

img_vis = omas_bf_read ([ s_fn '-vis*' $],-2$, 'double' $)$;

catch

$\%$ if this file does not exist, jump over it continue end spam $=$ spam_load([ spamdir

filesep s_fn '-*.spam' ]);

fprintf('Analysing \%s ... $\backslash n$ ', s_fn);

\% Calculate the threshold and then create the mask img_ir_mbg $=$ img_ir > params.bg_thresh;

thresh $=$ mean $\left(i m g \_i r\left(i m g \_i r>\right.\right.$ params.bg_thresh) $)$;

img_ir_mask $=$ img_ir $>$ thresh;

img_ir_erode $=$ imerode(img_ir_mask, strel_erode);

img_ir_mask =imdilate(img_ir_erode, strel_dilate);

if params.debug subplot $(1,4,[1])$;

imagesc(img_ir);

colormap('jet');

$\operatorname{subplot}(1,4,[2])$;

imagesc(img_ir_mbg);

$\operatorname{subplot}(1,4,[3])$;

imagesc(img_ir_erode);

subplot $(1,4,[\overline{4}])$;

imagesc(img_ir_mask);

input('next');

end img_vis_mask $=$ zeros(size(img_vis) $)$; 


\section{Appendix}

if params.r_bg $>0$ img_vis_bg $=$ spam_bg_gauss(img_vis, $5^{*}$ params.r_bg, true); else img_vis_bg = img_vis;

end for $\mathrm{j}=1$ :size(spam.events, 1$)$ pos $=\operatorname{round}(\operatorname{spam} . \operatorname{events}(\mathrm{j},[34])+.5)$;

[cnts, img_vis_mask] = get_intensity(pos,img_vis_bg, nhood_clust, img_vis_mask);

if cnts $>0$ if img_ir_mask $(\operatorname{pos}(1), \operatorname{pos}(2))>0$ total_in $=$ total_in +1 ;

sq_in $=$ sq_in $+\operatorname{cnts}^{\wedge} 2$; counts_in $=$ counts_in + cnts;

else total_out $=$ total_out $+1 ;$ sq_out $=$ sq_out $+\operatorname{cnts}^{\wedge} 2$; counts_out $=$ counts_out

+ cnts;

end

end

end

omas_bf_write([s_fn, '-masks.obf'], \{double(img_ir_mask), double(img_vis_mask) $)$

) ;

end

f_in $=$ total_in/(total_in + total_out $)$;

avg_in $=$ counts_in /total_in; avg_out $=$ counts_out $/$ total_out;

sigma_in $=\operatorname{sqrt}(($ sq_in/total_in - avg_in^2)/total_in $)$;

sigma_out $=\operatorname{sqrt}\left(\left(\mathrm{sq} \_\right.\right.$out $/$total_out - avg_out^2)/total_out $)$;

fprintf('fraction: $\backslash \mathrm{n} \backslash \mathrm{t}$ inside: $\% 2.2 \mathrm{f} \backslash \mathrm{n} \backslash$ toutside: $\% 2.2 \mathrm{f} \backslash \mathrm{n}$ ', f_in, 1-f_in);

fprintf('average counts: $\backslash \mathrm{n} \backslash \mathrm{t}$ inside:

$\% \mathrm{f}(+-\% \mathrm{f}) \backslash \mathrm{n} \backslash$ toutside:

\%f $(+-\% \mathrm{f}) \backslash \mathrm{n}$ ', avg_in, sigma_in, avg_out, sigma_out);

end

function $[\mathrm{i}$, slice_add $]=$ get_intensity (center, slice, nhood, slice_add) $\mathrm{s}=$ floor(size(nhood) $/ 2)$;

$\%$ left, top, right and bottom of the event $\mathrm{l}=\operatorname{round}(\operatorname{center}(1)-\mathrm{s}(1)) ; \mathrm{t}=\operatorname{round}(\operatorname{center}(2)$

$-\mathrm{s}(2))$;

$\mathrm{r}=\operatorname{round}(\operatorname{center}(1)+\mathrm{s}(1))$;

$\mathrm{b}=\operatorname{round}(\operatorname{center}(2)+\mathrm{s}(2))$;

if $(\mathrm{l}<1)\|(\mathrm{t}<1)\|(\mathrm{r}>\operatorname{size}($ slice, 1$)) \|(\mathrm{b}>\operatorname{size}($ slice, 2$)) \mathrm{i}=0$; return;

end

if nargout $>1$ slice_add(l:r, t:b) $=\max ($ slice_add(l:r, t:b), nhood $)$;

end

$\%$ right now I am adding rectangles event $=$ slice(l:r, t:b);

$\mathrm{i}=\operatorname{sum}\left(\operatorname{sum}\left(\right.\right.$ event $*^{*}$ nhood $\left.)\right)$;

end

\section{Preparation of C.elegans specimen (Section 3.4)}

Automated freeze substituion (AFS) The fixative was produced by dissolving $0.1 \%$ $\mathrm{KMnO}_{4}$ in $5 \%$ water. Afterwards $95 \%$ acetone are added. The specimen were incubated with the solution for $48 \mathrm{~h}$ at $-90^{\circ} \mathrm{C}$. (Longer incubation does not seem to influence the fluorescence level.) Afterward the specimen were thawed to $-20^{\circ} \mathrm{C}$ with a thawing rate 
of $5^{\circ} \mathrm{C} / \mathrm{h}$. Finally the specimen were kept at $-20^{\circ} \mathrm{C}$ for $2-8 \mathrm{~h}$. (Incubation time at $-20^{\circ} \mathrm{C}$ influences the fluorescence level-the shorter, the brighter.)

Washing and ethanol substitution The following steps were performed at $-20^{\circ} \mathrm{C}$ :

two times $30 \%$ ethanol, $65 \%$ acetone and $5 \%$ water for $1 \mathrm{~h}$,

two times $70 \%$ ethanol, $25 \%$ acetone and $5 \%$ water for $1 \mathrm{~h}$,

two times $95 \%$ ethanol and $5 \%$ water for $1 \mathrm{~h}$.

Infiltration with LR White The infiltration was performed at $-20^{\circ} \mathrm{C}$. Two solutions of either ethanol (in the following assigned as A) or LR White (in the following assigned as B) with $5 \%$ of water in each were produded and precooled to $-20^{\circ} \mathrm{C}$. The solution of LR White was at first stirred using a wooden stick, untill it geot clear and afterwards mixed with nitrogen gas. The polymerization was initialized chemically after walking through the following steps:

$30 \% \mathrm{~B}, 70 \% \mathrm{~A}$ and $0.05 \% \mathrm{LR}$ White accelerator for $5 \mathrm{~h}$,

two times $70 \% \mathrm{~B}, 30 \% \mathrm{~A}$ and $0.05 \%$ LR White accelerator for $6 \mathrm{~h}$,

two times $95 \%$ B, $5 \% \mathrm{~A}$ and $0.05 \%$ LR White accelerator for $1 \mathrm{~h}$

$95 \% \mathrm{~B}$ and $5 \% \mathrm{~A}$ over night (addition of accelerator would yield in polymerization), transfer of the samples into the embedding mold,

$95 \% \mathrm{~B}, 5 \% \mathrm{~A}$ and $0.05 \% \mathrm{LR}$ White accelerator for $2 \mathrm{~h}$,

two times $95 \%$ B, $5 \% \mathrm{~A}$ and $0.1 \%$ LR White accelerator for $1 \mathrm{~h}$.

Polymeriztaion of LR White The polymerization of the infiltrated specimen was performed by adding $95 \% \mathrm{~B}, 5 \% \mathrm{~A}$ and $0.15 \% \mathrm{LR}$ White accelerator for $24 \mathrm{~h}$ at $-20^{\circ} \mathrm{C}$. During polymerization the samples were covered with Aclar film to prevent the contamination with oxygen.

\section{Preparation of mouse tissue (Section 3.5)}

The used chemicals were received from Sigma Aldrich (St. Louis, MO, USA), except for PFA, LR White and Epon resin. The latter were obtained from TedPella (Redding, CA, USA).

LR White embedding Transgenic mice were heart perfused with of $4 \%$ PFA in $0.1 \mathrm{M}$ PBS. The desired tissue was then removed using sharp scissors and post-fixed for $24 \mathrm{~h}$ at $40^{\circ} \mathrm{C}$. The tissue was washed three times with PBS for $5 \mathrm{~min}$.

The samples were dehydrated and incubated with the monomer solution according to the following dilution series:

$50 \%$ ethanol for $10 \mathrm{~min}$,

$70 \%$ ethanol for $10 \mathrm{~min}$,

$95 \%$ ethanol for $10 \mathrm{~min}$,

$50 \%$ ethanol and $50 \%$ LR White $1 \mathrm{~h}$,

$30 \%$ ethanol and $70 \%$ LR White $1 \mathrm{~h}$, 


\section{Appendix}

three times pure resin for $1 \mathrm{~h}$,

pure resin over night at $4^{\circ} \mathrm{C}$.

The specimen were placed at the bottom of an embedding mold, which was then filled with LR White (medium grade) and polymerized for $14-48 \mathrm{~h}$ at $60^{\circ} \mathrm{C}$. The polymerized blocks were trimmed and cut using an custom-built ATUM unit, equipped with a diamond knife. Subsequently they were taken up on gelatin coated microscope glass slides. Afterwards they were sent to Göttingen. The slides were heated to $90^{\circ} \mathrm{C}$ on a heat plate before staining.

The post-embedding immuno-staining was performed according to the following protocol:

$50 \mathrm{mM}$ glycine in TRIS buffer $(50 \mathrm{mM})$ for $5 \mathrm{~min}$,

blocking solution of $1 \%$ BSA in TRIS buffer for $15-30 \mathrm{~min}$,

incubation with primary antibody (rabbit-anti-GFP, Milipore GmbH, Schwalbach,

Germany, $1: 100$ in blocking solution) over night at room temperature,

three times washing with TRIS buffer for $1 \mathrm{~min}$,

incubation with secondary antibody (Dianova GmbH, Hamburg, Germany) coupled to ATTO590 or ATTO532 (ATTO-TEC GmbH, Siegen, Germany) in blocking solution ( $1: 100)$ for $1-2 \mathrm{~h}$ at room temperature,

three times washing with TRIS buffer for $1 \mathrm{~min}$.

The sections were subsequently embedded in mowiol.

Epon embedding Postnatal day 0 pups were anesthetized using $0.1 \mathrm{ml}$ pentobarbital. Afterwards they were heart perfused with $50 \mathrm{ml}$ of $2 \%$ PFA in $0.1 \mathrm{M}$ PBS. The desired tissue was then removed using sharp scissors and post-fixed for $30 \mathrm{~min}$.

In case of pre-embedding staining, the tissue was washed two times with PBS and for $24 \mathrm{~h}$ incubated with a solution of $4 \%$ BSA and $1 \%$ Triton in PBS. Afterwards, the primary antibody (rabbit, anti-GFP, Chemicon) is diluted to $1 / 300$ and added on the tissue for $3 \mathrm{~d}$. After three times washing with PBS for 5 min each, the tissue was incubated with the secondary antibody (Dianova GmbH, Hamburg, Germany), which previously had been coupled to ATTO590 (ATTO-TEC GmbH, Siegen, Germany) in PBS, containing $1 \%$ BSA and Triton respectively for one day. Subsequently the tissue was again washed three times with PBS and post-fixed for $1 \mathrm{~h}$.

The either stained or untreated tissue was again washed with PBS for three times (each time up to $5 \mathrm{~min}$ ) and incubated with epon resin by the following dilution series:

two times $50 \%$ ethanol for $15 \mathrm{~min}$,

$70 \%$ ethanol for $15 \mathrm{~min}$,

$95 \%$ ethanol for $15 \mathrm{~min}$,

two times $100 \%$ ethanol for $15 \mathrm{~min}$,

$50 \%$ ethanol and $50 \%$ propylenoxid for $30 \mathrm{~min}$,

$100 \%$ propylenoxid for $30 \mathrm{~min}$,

$50 \%$ propylenoxid and $50 \%$ epon resin for $2 \mathrm{~h}$,

three times pure resin for $2 \mathrm{~h}$ and one time for $24 \mathrm{~h}$. 
Afterwards the tissue was placed into fresh-made resin, which was then polymerized for $48 \mathrm{~h}$ at $60^{\circ} \mathrm{C}$.

The polymerized blocks were trimmed and cut using an custom-built ATUM unit, equipped with a diamond knife.

The sections were collected automatically on lumox tape.

In case of post-embedding staining the sections are treated with solution of $15 \mathrm{~g} \mathrm{NaOH}$ in $100 \mathrm{ml}$ ethanol. Afterwards they were hydrated with a descending alcohol series to $50 \%$ ethanol and subsequently immersed in TRIS buffer for $10 \mathrm{~min}$. Then they were blocked with $1 \%$ BSA in TRIS and incubated with the primary antibody (anti-GFP, Chemicon, $1 / 300$ in blocking solution) for $24 \mathrm{~h}$ at $4^{\circ} \mathrm{C}$. After several washing steps with TRIS buffer, the same secondary antibody as above was added for $2-4 \mathrm{~h}(1 / 300$ in blocking solution). Finally, the sections were washed three times with TRIS buffer and mounted in Vectashield to be sent to Göttingen. 



\section{Bibliography}

[1] P. R. Lewis A. M. Glauert, editor. Biological Specimen Preparation for Transmission Electron Microscopy. Portland Press, 1998.

[2] E. Abbe. Beiträge zur Theorie des Mikroskops und der mikroskopischen Wahrnehmung. Arch. Mikr. Anat., 9:413-468, 1873.

[3] M. Andresen, A.C. Stiel, J. Fölling, D. Wenzel, A. Schönle, A. Egner, C. Eggeling, S.W. Hell, and S. Jakobs. Photoswitchable fluorescent proteins enable monochromatic multilabel imaging and dual color fluorescence nanoscopy. Nat. Biotech., 26 (9):1035-1040, 2008. ISSN 1087-0156.

[4] D. Axelrrod and et al. Total internal reflection fluorescence. In J.R. Lakowicz, editor, Topics in Fluorescence Spectroscopy: Biochemical Applications, volume 3. Plenum Press, New York, 1992.

[5] D. Bachhuber, K.; Froesch. Melamine resins, a new class of water-soluble embedding media for electron microscopy. J. Microsc., 130:1-9, 1983.

[6] M. W. Bagnall, R. J. Stevens, and S. du Lac. Transgenic mouse lines subdivide medial vestibular nucleus neurons into discrete, neurochemically distinct populations. J. Neurosci., 27(9):2318-2330, 2007.

[7] C.I. Bargmann and H.R. Horvitz. Chemosensory neurons with overlapping functions direct chemotaxis to multiple chemicals in c.elegans. Neuron, 7:729-742, 1991.

[8] E. Bartova, J. Krejci, A. Harnicarova, G. Galiova, and S. Kozubek. Histone modifications and nuclear architecture: A review. J. Histochem. Cytochem., 56(8): 711-721, 2008.

[9] M. K. Bennett, J. E. Garcia-Arrarás, L. A. Elferink, K. Peterson, A. M. Fleming, C. D. Hazuka, and R. H. Scheller. The syntaxin family of vesicular transport receptors. CELL, 74(5):863-873, 1993. ISSN 0092-8674.

[10] J. Bereiter-Hahn and M. Vöth. Dynamics of mitochondria in living cells: Shape changes, dislocations, fusion, and fission of mitochondria. Microsc. Res. Techniq., 27(3):198-219, 1994.

[11] E. Betzig, G.H. Patterson, R. Sougrat, O.W. Lindwasser, S. Olenych, J.S. Bonifacino, M.W. Davidson, J. Lippincott-Schwartz, and H.F. Hess. Imaging intracellular fluorescent proteins at nanometer resolution. Science, 313(5793):1642-1645, 2006. 
[12] J. Bewersdorf, A. Egner, and S. W. Hell. 4pi microscopy. In J. Pawley, editor, Handbook of Biological Confocal Microscopy, pages 561-570. Springer, New York, 2006.

[13] J. Bewersdorf, R. Schmidt, and S. W. Hell. Comparison of I5M and 4Pi-microscopy. J. Microsc., 222:105-117, 2006.

[14] H. Bock, C. Geisler, C. A. Wurm, S. Jakobs, A. Schoenle, A. Egner, S. W. Hell, and C. Eggeling. Two-color far-field fluorescence nanoscopy based on photoswitching emitters. Appl. Phys. B, 88(161-165), 2007.

[15] M. Born and E. Wolf. Principles of Optics. Cambridge University Press, Cambridge, New York, Melbourne, Madrid, Cape Town, 7th edition, 2002.

[16] M. Bossi, J. Fölling, V. N. Belov, V. P. Boyarskiy, R. Medda, A. Egner, C. Eggeling, A. Schoenle, and S. W. Hell. Multicolor far-field fluorescence nanoscopy through isolated detection of distinct molecular species. Nano Lett., 8(8):2463-2468, 2008. ISSN 1530-6984.

[17] S. Brenner. The genetics of Caenorhabditis elegans. Genetics, 77:71-94, 1974.

[18] S. Bretschneider, C. Eggeling, and S. W. Hell. Breaking the diffraction barrier in fluorescence microscopy by optical shelving. Phys. Rev. Lett., 98:218103, 2007.

[19] L. Broglie. La nouvelle dynamique des quanta. Électrons et Photons: Rapports et Discussions du Cinquième Conseil de Physique, 1928.

[20] E. Carlemalm. Lowicryl resins in microbiology. J. Struct. Biol., 104(1-3):189-191, 1990. ISSN 1047-8477.

[21] M. Chalfie, Y. Tu, G. Euskirchen, W. W. Ward, and D. C. Prasher. Green fluorescent protein as a marker for gene expression. Science, 263:802-804, 1994.

[22] N. Davidson. Sky Phenomena: A Guide to Naked Eye Observation of the Heavens. Floris Books, Edinburgh, 1993.

[23] W. Denk, J. H. Strickler, and W. W. Webb. Two-photon laser scanning fluorescence microscopy. Science, 248:73-76, 1990.

[24] G. Donnert, J. Keller, R. Medda, M. A. Andrei, S. O. Rizzoli, R. Lührmann, R. Jahn, C. Eggeling, and S. W. Hell. Macromolecular-scale resolution in biological fluorescence microscopy. P. Natl. Acad. Sci. U.S.A., 103(31):11440-11445, 2006.

[25] G. Donnert, C. Eggeling, and S. W. Hell. Major signal increase in flurorescence microscopy through dark-state relaxation. Nat. Methods, 4(1):81-86, 2007.

[26] G. Donnert, J. Keller, C. A. Wurm, S. O. Rizzoli, V. Westphal, A. Schönle, R. Jahn, S. Jakobs, C. Eggeling, and S. W. Hell. Two-color far-field fluorescence nanoscopy. Biophys. J., 92(8):L67-69L, 2007. 
[27] E. van Donselaar, G. Posthuma, D. Zeuschner, J. W. Slot, and B. M. Humbel. Immunogold labeling of cryo-sections from high-pressure frozen cells. Microsc. Microanal., 13:168-169, 2007.

[28] M. Dyba and S.W. Hell. Focal spots of size 1/23 open up far-field fluorescence microscopy at $33 \mathrm{~nm}$ axial resolution. Phys. Rev. Lett., 88:163901, 2002.

[29] M. Dyba, J. Keller, and S. W. Hell. Phase filter enhanced STED-4Pi fluorescence microscopy: theory and experiment. New J. Phys., 7, 2005.

[30] A. Egner and S. W. Hell. Fluorescence microscopy with super-resolved optical sections. Trends Cell. Biol., 15(4):207-215, 2005.

[31] A. Egner, C. Geisler, C. von Middendorff, H. Bock, D. Wenzel, R Medda, M. Andresen, A.C. Stiel, S. Jakobs, C. Eggeling, A. Schonle, and S.W. Hell. Fluorescence nanoscopy in whole cells by asnychronous localization of photoswitching emitters. Biophys. J., 93:3285-3290, 2007.

[32] A. Einstein. Zur Quantentheorie der Strahlung. Physik. Zeitschr., 18:121-128, 1917.

[33] R. B. Fasshauer, D.and Sutton, A. T. Brunger, and R. Jahn. Conserved structural features of the synaptic fusion complex: SNARE proteins reclassified as Q- and R-SNAREs. P. Nat. Acad. Sci. U.S.A., 95(26):15781-15786, 1998.

[34] G. Feng, R. H. Mellor, M. Bernstein, C. Keller-Peck, Q. T. Nguyen, M. Wallace, J. M. Nerbonne, J. W. Lichtman, and J. R. Sanes. Imaging Neuronal Subsets in Transgenic Mice Expressing Multiple Spectral Variants of GFP. Neuron, 28(1): 41-51, 2000. ISSN 0896-6273.

[35] M. Fernandez-Suarez and A.Y. Ting. Fluorescent probes for super-resolution imaging in living cells. Nat. Rev. Mol. Cell. Biol., 9(12):929-943, 2008. ISSN 1471-0072.

[36] J. Fölling, V. Belov, R. Kunetsky, R. Medda, A. Schönle, A. Egner, C. Eggeling, M. Bossi, and S. W. Hell. Photochromic rhodamines provide nanoscopy with optical sectioning. Angew. Chem. Int. Ed., 46(33):6266-6270, 2007.

[37] J. Fölling, V. Belov, D. Riedel, A. Schönle, A. Egner, C. Eggeling, M. Bossi, and S. W. Hell. Fluorescence nanoscopy with optical sectioning by two-photon induced molecular switching using continuous-wave lasers. Chem. Phys. Chem., 9:321-326, 2008 .

[38] J. Fölling, M. Bossi, H. Bock, R. Medda, C.A. Wurm, B. Hein, S. Jakobs, C. Eggeling, and S.W. Hell. Fluorescence nanoscopy by ground-state depletion and single-molecule return. Nat. Methods, 5(11):943-945, 2008. ISSN 1548-7091.

[39] T. G. Frey and C. A. Mannella. The internal structure of mitochondria. Trends Biochem. Sci., 25(7):319-324, 2000. ISSN 0968-0004. 
[40] C. Frokjaer-Jensen, M.W. Davis, C. E. Hopkins, B. J. Newman, J. M. Thummel, S.-P. Olesen, M. Grunnet, and E. M. Jorgensen. Single-copy insertion of transgenes in Caenorhabditis elegans. Nat. Genet., 40(11):1375-1383, 2008. ISSN 1061-4036.

[41] D. Frösch and C. Westphal. Melamine resins and their application in electron microscopy. Electron. Microsc. Rev., 2(2):231-255, 1989. ISSN 0892-0354.

[42] E. Fuchs and K. Weber. Intermediate filaments: Structure, dynamics, function and disease. Annu. Rev. Biochem., 63(1):345-382, 1994.

[43] J. F. Gennaro, W. L. Nastuk, and D. T. Rutherford. Reversible depletion of synaptic vesicles induced by application of high external potassium to the frog neuromuscular junction. J. Physiol., 280(1):237-247, 1978.

[44] D. Gerlach. Geschichte der Mikroskopie. 2009.

[45] F.J. Giessibl. Advances in atomic force microscopy. Rev. Mod. Phys., 75(3):949983, 2003.

[46] A. Giske. CryoSTED microscopy: A new spectroscopic approach for improving the resolution of STED microscopy using low temperature. University of Heidelberg, Germany, 2007.

[47] S. Glaschick, C. Räcker, K. Deuschle, J. Wiedenmann, F. Oswald, V. Mailänder, and G. Nienhaus. Axial resolution enhancement by $4 \mathrm{Pi}$ confocal fluorescence microscopy with two-photon excitation. J. Biol. Phys., 33(5):433-443, 2007.

[48] A.M. Glauert and R.H. Glauert. J. Biophys. Biochem. Cy., 4:191, 1958.

[49] U. M. Glauert, G. E. Rogers, and R. H. Glauert. A new embedding medium for electron microscopy. Nature, 178:803, 1956.

[50] D. Gook, S. M. Osborn, H. Bourne, D. H. Edgar, and A. L. Speirs. Fluorescent study of chromatin and tubulin in apparently unfertilized human oocytes following icsi. Mol. Hum. Reprod., 4:1130-1135, 1998.

[51] J. W. Gordon and F. H. Ruddle. Integration and stable germ line transmission of genes injected into mouse pronuclei. Science, 214(4526):1244-1246, 1981.

[52] A. Gottschalk and W. R. Schafer. Visualization of integral and peripheral cell surface proteins in live Caenorhabditis elegans. J. Neurosci. Meth., 154(1-2):6879, 2006.

[53] J.A. Hobot G.R. Newman. Resin Microscopy and On-Section Immunocytochemistry. Springer-Verlag, 1993.

[54] E. J. Gualda, G. Filippidis, G. Voglis, M. Mari, C. Fotakis, and N. Tavernarakis. In vivo imaging of cellular structures in Caenorhabditis elegans by combined TPEF, SHG and THG microscopy. J .Microsc.-Oxford, 229(1):141-150, 2008. 
[55] M. G. L. Gustafsson. Surpassing the lateral resolution limit by a factor of two using structured illumination microscopy. J. Microsc., 198(2):82-87, 2000.

[56] M. G. L. Gustafsson, D. A. Agard, and J. W. Sedat. I5M: 3D widefield light microscopy with better than 100nm axial resolution. J. Microsc., 195:10-16, 1999.

[57] D.H. Hall and R.L. Russell. The posterior nervous system of the nematode Caenorhabditis elegans: serial reconstruction of identified neurons and complete pattern of synaptic interactions. J. Neurosci., 11(1):1-22, 1991.

[58] B. Harke, J. Keller, C. K. Ullal, V. Westphal, A. Schoenle, and S. W. Hell. Resolution scaling in sted microscopy. Opt. Express, 16(6):4154-4162, 2008.

[59] B. Harke, J. Ullal, J. Keller, and S. W. Hell. Three-dimensional nanoscopy of colloidal crystals. Nano Lett., 8 (5):1309-1313, 2008.

[60] T. W. Harris, K. Schuske, and E. M. Jorgensen. Studies of Synaptic Vesicle Endocytosis in the Nematode C. elegans. Traffic, 2(9):597-605, 2001.

[61] K. J. Hayworth, N. Kasthuri, R. Schalek, and J. W. Lichtman. Automating the collection of ultrathin serial sections for large volume TEM reconstructions. Microsc. Microanal., 12:86-87, 2006.

[62] A.A. Heikal, S.T. Hess, G.S. Baird, R.Y. Tsien, and W.W. Webb. Molecular spectroscopy and dynamics of intrinsically fluorescent proteins: Coral red (dsRed) and yellow (Citrine). P. Natl. Acad. Sci. U.S.A., 97(22):11996-12001, 2000.

[63] M. Heilemann, S. van de Linde, M. Schüttpelz, R. Kasper, B. Seefeldt, A. Mukherjee, P. Tinnefeld, and M. Sauer. Subdiffraction-resolution fluorescence imaging with conventional fluorescent probes. Angew. Chem. Int. Ed., 47(33):6172-6176, 2008 .

[64] B. Hein, K.I. Willig, and S.W. Hell. Stimulated emission depletion (STED) nanoscopy of a fluorescent protein-labeled organelle inside a living cell. P. Nat. Acad. Sci. U.S.A., 105(38):14271-14276, 2008.

[65] R. Heintzmann, T.M. Jovin, and C. Cremer. Saturated patterned excitation microscopy - a concept for optical resolution improvement. J. Opt. Soc. Am. A, 19 (8):1599-1609, 2002.

[66] S. W. Hell. Double-scanning confocal microscope, 1990. US Patent, No 0491289.

[67] S. W. Hell and M. Kroug. Ground-state depletion fluorescence microscopy, a concept for breaking the diffraction resolution limit. Appl. Phys. B, 60:495-497, 1995.

[68] S. W. Hell and A. Schönle. Nanoscale resolution in far-field fluorescence microscopy. In P. W Hawkes Spence and J. C. H., editors, Science of Microscopy, volume 2, pages $790-834$. Springer, 2006. 
Bibliography

[69] S. W. Hell and J. Wichmann. Breaking the diffraction resolution limit by stimulated emission: stimulated emission depletion microscopy. Opt. Lett., 19(11):780-782, 1994.

[70] S. W. Hell, R. Schmidt, and A. Egner. Diffraction-unlimited three-dimensional optical nanoscopy with opposing lenses. Nat. Photon., 3(7):381-387, 2009. ISSN $1749-4885$.

[71] Stefan W Hell. Microscopy and its focal switch. Nat. Methods, 6(1):24-32, 2009. ISSN 1548-7091.

[72] S.W. Hell. Toward fluorescence nanoscopy. Nat. Biotech., 21(11):1347-1355, 2003.

[73] S.W. Hell, M. Schrader, K. Bahlmann, F. Meinecke, and C. Cremer. Realisation of depletion by stimulated emission in fluorescence microscopy. In P.C. Cheng, P.P. Hwang, J.L. Wu, G. Wang, and H. Kim, editors, Focus on Multidimensional Microscopy, volume 1, pages 78-88. World Scientific, Singapore, 1999.

[74] M. Helmstädter, K. L. Briggman, and W. Denk. 3D structural imaging of the brain with photons and electrons. Curr. Opin. Neurobiol., 18(6):633-641, 2008. ISSN 0959-4388.

[75] S.T. Hess, T.P.K. Girirajan, and M.D. Mason. Ultra-high resolution imaging by fluorescence photoactivation localization microscopy. Biophys. J., 91(11):42584272,2006

[76] J. E. Heuser and T. S. Reese. Evidence for recycling of synaptic vesicle membrane during transmitter release at the frog neuromuscular junction. J. Cell Biol., 57(2): 315-344, 1973.

[77] J. Hill. The Construction of Timer, from its early growth; Explained by Microscope, and proven from Experiments, in a great Variety of Kinds. 1770.

[78] J. Hillier and R. F. Baker. Microanalysis by means of electrons. J. Appl. Phys., 15 (9):663-675, 1944.

[79] S. Hillmer, S. Joachim, and D. G. Robinson. Rapid polymerization of LR-White for immunocytochemistry. Histochem. Cell. Biol., 95(3):315-318, 1991.

[80] W. His. Beschreibung eines Mikrotoms. Arch. Mikr. Anat., 6(1):229-232, 1870.

[81] O. Hobert and P. Loria. Uses of GFP in Caenorhabditis elegans. In Steven R. Kain Martin Chalfie, editor, Green Fluorescent Protein (Second Edition), pages 203-226. 2005 .

[82] J. Hodgkin, H. R. Horvitz, B. R. Jasny, and J. Kimble. C. elegans: Sequence to biology. Science, 282(5396):2011, 1998. 
[83] M. Hofmann, C. Eggeling, S. Jakobs, and S.W. Hell. Breaking the diffraction barrier in fluorescence microscopy at low light intensities by using reversibly photoswitchable proteins. P. Natl. Acad. Sci. U.S.A., 102(49):17565-17569, 2005.

[84] http://www.wormatlas.org/.

[85] http://www.wormbase.org/.

[86] B. Huang, W. Wang, M. Bates, and X. Zhuang. Three-dimensional super-resolution imaging by stochastic optical reconstruction microscopy. Science, 319:810-813, 2008.

[87] G. L. Humason. Animal tissue techniques. San Francisco, W.H. Freeman, 1962.

[88] M. Ikawa, K. Kominami, Y. Yoshimura, K. Tanaka, Y. Nishimune, and M. Okabe. Green fluorescent protein as a marker in transgenic mice. Dev.Growth Differ., 37 (4):455-459, 1995.

[89] H. Ishikawa, J. Usukura, and E. Yamada. Application of cryomicrotomy to the rapid-freeze, deep-etch replica method for unfixed tissues and cells. J. Electron. Microsc., 31(2):198-201, 1982.

[90] R. Jaenisch, J. Dausman, V. Cox, and H. Fan. Infection of developing mouse embryos with murine leukemia virus: tissue specificity and genetic transmission of the virus. Hamatol. Bluttransfus., 19:341, 1976.

[91] R. Jahn and R. H. Scheller. SNAREs - engines for membrane fusion. Nat. Rev. Mol. Cell Biol., 7(9):631-643, 2006.

[92] R. Jahn, W. Schiebler, C. Ouimet, and P. Greengard. A 38,000-dalton membrane protein (p38) present in synaptic vesicles. P. Natl. Acad. Sci. U.S.A., 82:4137-4141, 1985 .

[93] Reinhard Jahn and Richard H. Scheller. Nat. Rev. Mol. Cell Biol., 7(9):631-643, 2006.

[94] S. Jakobs. High resolution imaging of live mitochondria. BBA - Mol. Cell Res., 1763(5-6):561-575, 2006. ISSN 0167-4889.

[95] M.F. Juette, T.J. Gould, M.D. Lessard, M.J. Mlodzianoski, B.S. Nagpure, B.T. Bennett, S.T. Hess, and J. Bewersdorf. Three-dimensional sub-100 nm resolution fluorescence microscopy of thick samples. Nat. Methods, 5(6):527-529, 2008. ISSN 1548-7091.

[96] N. Jung and V. Haucke. Clathrin-mediated endocytosis at synapses. Traffic, 8(9): 1129-1136, 2007.

[97] H. Kaczmarek and C. Decker. Interpenetrating polymer networks. I. Photopolymerization of multiacrylate systems. J. Appl. Polym. Sci., 54(13):2147-2156, 1994. 
[98] S. Kawata, Y. Inouye, and P. Verma. Plasmonics for near-field nano-imaging and superlensing. Nat. Photon., 3(7):388-394, 2009. ISSN 1749-4885.

[99] D. R. Keene, S. F. Tufa, G. P. Lunstrum, P. Holden, and W. A. Horton. Confocal/TEM Overlay Microscopy: A Simple Method for Correlating Confocal and Electron Microscopy of Cells Expressing GFP/YFP Fusion Proteins. Microsc. Microanal., 14(04):342-348, 2008.

[100] J. Keller, A. Schönle, and S.W. Hell. Efficient fluorescence inhibition patterns for resolft microscopy. Opt. Express, 15(6):3361-3371, 2007.

[101] A.I. Kirkland and L Hutchinsons. Atomic resolution transmission electron microscopy. In P.W. Hawkes and J.C. Spence, editors, Science of Microscopy, volume 1, pages 3-64. Springer, 2007.

[102] A. Kirsch, C. Meyer, and T. M. Jovin. Integrating of optical techniques in scanning probe microscopes; the scanning near-field optical microscope (snom). In E. Kohen and J. G. Hirschberg, editors, Analytical Use of Fluorescenct Probes in Oncology, pages 317-323. Plenum Press, New York, 1996.

[103] T. A. Klar, S. Jakobs, M. Dyba, A. Egner, and S. W. Hell. Fluorescence microscopy with diffraction resolution limit broken by stimulated emission. P. Nat. Acad. Sci. U.S.A, 97:8206-8210, 2000.

[104] T.A. Klar and S.W. Hell. Subdiffraction resolution in far-field fluorescence microscopy. Opt. Lett., 24(14):954-956, 1999.

[105] M. Knoll and E. Ruska. Das Elektronenmikroskop. Z. Phys., 78:318-339, 1932.

[106] J. Blum K.R. Porter. Anat. Rec., 117:685-710, 1953.

[107] J. R. Kremer, D. N. Mastronarde, and J. R. McIntosh. Computer Visualization of Three-Dimensional Image Data Using IMOD. J. Struct. Biol., 116(1):71-76, 1996.

[108] J. Kuo, editor. Electron Microscopy. Methods and Protocols, volume 369 of Methods in Molecular Biology. Springer Protocols, 2007.

[109] H. Kushida. A modification of the water-miscible epoxy resin "Durcupan" embedding method for ultrathin sectioning. J. Electron. Microsc., 12(1):72-73, 1963.

[110] J. R. Lakowicz. Principles of fluorescence spectroscopy. Kluwer Academic/Plenum, New York, 1999.

[111] P. Lemmer, M. Gunkel, D. Baddeley, R. Kaufmann, A. Urich, Y. Weiland, J. Reymann, P. Möller, M. Hausmann, and C. Cremer. SPDM: light microscopy with single-molecule resolution at the nanoscale. App. Phys. B, 93(1):1-12, 2008.

[112] J. W. Lichtman and J. R. Sanes. Ome sweet ome: what can the genome tell us about the connectome? Curr. Opin. Neurobiol., 18(3):346-353, 2008. ISSN 0959-4388. 
[113] J. W. Lichtman, J. Livet, and J. R. Sanes. A technicolour approach to the connectome. Nat. Rev. Neurosci., 9(6):417-422, 2008. ISSN 1471-003X.

[114] A. D. Linstedt and H. P. Hauri. Giantin, a novel conserved Golgi membrane protein containing a cytoplasmic domain of at least $350 \mathrm{kDa}$. Mol. Biol. Cell, 4(7):679-693, 1993.

[115] W. Liou, H. J. Geuze, and J. W. Slot. Improving structural integrity of cryosections for immunogold labeling. Histochem. Cell. Biol., 106(1):41-58, 1996.

[116] J. Livet, T. A. Weissman, H. Kang, R. W. Draft, J. Lu, R. A. Bennis, J. R. Sanes, and J. W. Lichtman. Transgenic strategies for combinatorial expression of fluorescent proteins in the nervous system. Nature, 450(7166):56-62, 2007. ISSN 0028-0836.

[117] K. Luby-Phelps, G. Ning, J. Fogerty, and J. C. Besharse. Visualization of identified GFP-expressing cells by light and electron microscopy. J. Histochem. Cytochem., 51(3):271-274, 2003.

[118] J. H. Luft. J. Biophys. Biochem. Cytol., 9:409, 1961.

[119] C.A. May, editor. Epoxy resins: chemistry and technolog, volume 2. 1988.

[120] E. Mayr. Die Entwicklung der biologischen Gedankenwelt. Springer, 2002.

[121] G. D. McPhail, T. Finn, and P. G. Isaacson. A useful low temperature method for post-embedding electron immunocytochemistry in routine histopathology. $J$. Pathol., 151(3):231-238, 1987.

[122] C. C. Mello, J. M. Kramer, D. Stinchcomb, and V. Ambros. Efficient gene-transfer In C.elegans - extrachromosomal maintenance and Integration of transforming sequences. E.M.B.O. J., 10(12):3959-3970, 1991.

[123] L. Melville, S. Dickson, M. L. Farquhar, S. E. Smith, and R. L. Peterson. Visualization of mycorrhizal fungal structures in resin embedded tissues with xanthene dyes using laser scanning confocal microscopy. Can. J. Bot., 76:174-178, 1998.

[124] L. Meyer, D. Wildanger, R. Medda, A. Punge, S. O. Rizzoli, G. Donnert, and S. W. Hell. Dual-color STED microscopy at $30 \mathrm{~nm}$ focal plane resolution. Small, 4(8): 1095-1100, 2008.

[125] K.D. Micheva and S.J. Smith. Array tomography: A new tool for imaging the molecular architecture and ultrastructure of neural circuits. Neuron, 55(1):25-36, 2007.

[126] M. Minsky. Microscopy apparatus, US Patent, No 3013467, 1961.

[127] P. J. Mintz and D. L. Spector. Compartmentalization of RNA processing factors within nuclear speckles. J. Struct. Biol., 129(2-3):241-251, 2000. 
Bibliography

[128] D.J. Müller and K. Anderson. Biomolecular imaging using atomic force microscopy. Trends Biotechnol., 20:S45-S49, 2002.

[129] G. Moneron and S. W. Hell. Two-photon excitation STED microscopy. Opt. Express, 17(17):14567-14573, 2009.

[130] A. Monneron and W. Bernhard. Fine structural organization of interphase nucleus in some mammalian cells. J. Ultra. Mol. Struct. R., 27(3-4):266, 1969.

[131] D. B. Murphy. Fundamentals of light microscopy and electronic imaging. WileyLiss, Inc., New York, 2001.

[132] M. L. Nonet. Visualization of synaptic specializations in live C. elegans with synaptic vesicle protein-GFP fusions. J. Neurosci. Meth., 89(1):33-40, 1999. ISSN 01650270 .

[133] M. Osborn, R. E. Webster, and K. Weber. Individual microtubules viewed by immunofluorescence and electron microscopy in the same PtK2 cell. J. Cell Biol., 77(3):R35-R42, 1978.

[134] S. R. P. Pavani, M. A. Thompson, J. S. Biteen, S. J. Lord, N. Liu, R. J. Twieg, R. Piestun, and W. E. Moerner. Three-dimensional, single-molecule fluorescence imaging beyond the diffraction limit by using a double-helix point spread function. P. Natl. Acad. Sci. U.S.A., 106(9):2995-2999, 2009.

[135] James B. Pawley, editor. Handbook of biological confocal microscopy. Springer Verlag, 3rd edition, 2006.

[136] D. W. Pohl, W. Denk, and M. Lanz. Optical stethoscopy: Image recording with resolution 1/20. Appl. Phys. Lett., 44:651-653, 1984.

[137] A. Pombo, M. Hollinshead, and P. R. Cook. Bridging the resolution gap: Imaging the same transcription factories in cryosections by light and electron microscopy. J. Histochem. Cytochem., 47(4):471-480, 1999.

[138] W.G. Potter. The chemistry and uses of epoxide resins. Trans. Ass. industr.med. Offrs., 13:42, 1963.

[139] J. Quekett. Practical treatise on the use of the microscope, including the different methods of preparing and examining animal, vegetable and mineral substances. London, Baillière, 1848.

[140] D. A. Richards, S. O. Rizzoli, and W. J. Betz. Effects of wortmannin and latrunculin a on slow endocytosis at the frog neuromuscular junction. J. Physiol., 557(1):77-91, 2004.

[141] S. O. Rizzoli and W. J. Betz. Synaptic vesicle pools. Nat. Rev. Neurosci., 6(1): 57-69, 2005. 
[142] S. O. Rizzoli and R. Jahn. Kiss-and-run, collapse and 'readily retrievable' vesicles. Traffic, 8(9):1137-1144, 2007.

[143] S. O. Rizzoli, I. Bethani, D. Zwilling, D. Wenzel, T. J. Siddiqui, D. Brandhorst, and R. Jahn. Evidence for early endosome-like fusion of recently endocytosed synaptic vesicles. Traffic, 7(9):1163-1176, 2006.

[144] M.J. Rust, M. Bates, and X. Zhuang. Sub-diffraction-limit imaging by stochastic optical reconstruction microscopy (STORM). Nat. Methods, 3:793-796, 2006.

[145] M. Saraste. Oxidative phosphorylation at the fin de siecle. Science, 283(5407): 1488, 1999. ISSN 00368075.

[146] C. Scala, G. Cenacchi, C. Ferrari, G. Pasquinelli, P. Preda, and G.C. Manara. A new acrylic resin formulation: a useful tool for histological, ultrastructural, and immunocytochemical investigations. J. Histochem. Cytochem., 40(11):1799-1804, 1992.

[147] R. Schmidt, C.A. Wurm, S. Jakobs, J. Engelhardt, A. Egner, and S.W. Hell. Spherical nanosized focal spot unravels the interior of cells. Nat. Methods, 5(6):539-544, 2008. ISSN 1548-7091.

[148] R. Schmidt, C. A. Wurm, A. Punge, A. Egner, S. Jakobs, and S. W. Hell. Mitochondrial cristae revealed with focused light. Nano Lett., 9(6):2508-2510, 2009. ISSN 1530-6984.

[149] A. Schönle. PSF engineering in fluorescence spectroscopy. Dissertation, RuprechtKarls-Universität, 2003.

[150] A. Schönle. Imspector, 2006.

[151] M. Schwentker, H. Bock, M. Hofmann, S. Jakobs, J. Bewersdorf, C. Eggeling, and S. W. Hell. Wide-field subdiffraction resolft microscopy using fluorescent protein photoswitching. Micr. Res. Techniq., 70(3):269-280, 2007.

[152] L. Shao, B. Isaac, S. Uzawa, D. A. Agard, J. W. Sedat, and M. G.L. Gustafsson. I5S: Wide-Field Light Microscopy with 100-nm-Scale Resolution in Three Dimensions. Biophys. J., 94(12):4971-4983, 2008. ISSN 0006-3495.

[153] H. Shroff, C.G Galbraith, J. A Galbraith, and E. Betzig. Live-cell photoactivated localization microscopy of nanoscale adhesion dynamics. Nat. Methods, 5(5):417423, 2008. ISSN 1548-7091.

[154] G. Shtengel, J.A. Galbraith, C.G. Galbraith, J. Lippincott-Schwartz, J.M. Gillette, S. Manley, C.M. Sougrat, R. Waterman, P. Kanchanawong, M.W. Davidson, R.D. Fetter, and H.F. Hess. Interferometric fluorescent super-resolution microscopy resolves 3d cellular ultrastructure. P. Nat. Acad. Sci. U.S.A., 106(9):3125-3130, 2009 . 
[155] J. J. Sieber, K. I. Willig, C. Kutzner, C. Gerding-Reimers, B. Harke, G. Donnert, B. Rammner, C. Eggeling, S. W. Hell, H. Grubmüller, and T. Lang. Anatomy and dynamics of a supramolecular membrane protein cluster. Science, 317:1072 - 1076, 2007.

[156] P. A. Sims and J. D. Hardin. Fluorescence-integrated transmission electron microscopy images. volume 369, pages 291-308. 2007.

[157] E. M. Slater and H. S. Slater. Light and electron microscopy. Cambridge University Press, London, 1993.

[158] J. A. Smeitink, M. Zeviani, D. M. Turnbull, and H. T. Jacobs. Mitochondrial medicine: A metabolic perspective on the pathology of oxidative phosphorylation disorders. Cellvb Metab., 3(1):9-13, January 2006. ISSN 1550-4131.

[159] A.R. Spurr. A low-viscosity epoxy resin embedding medium for electron microscopy. J. Ultrastruct. Res., 26(1):31-43, 1969.

[160] W. Staeubli. Compt. rend. Acad. sc., 250:1137, 1960.

[161] T. Staudt, M. Lang, R. Medda, J. Engelhardt, and S. W. Hell. 2,2'-thiodiethanol: a new water soluble mounting medium for high resolution optical microscopy. $\mathrm{Mi}$ crosc. Res. Techniq., 70:1-9, 2007.

[162] C. Steinhauer, C. Forthmann, J. Vogelsang, and P. Tinnefeld. Superresolution microscopy on the basis of engineered dark states. J. Am. Chem. Soc., 130(50): 16840-16841, 2008.

[163] A.C. Stiel, M. Andresen, H. Bock, M. Hilbert, A. Schilde, J.and Schönle, C. Eggeling, A. Egner, S.W. Hell, and S. Jakobs. Generation of monomeric reversibly switchable red fluorescent proteins for far-field fluorescence nanoscopy. Biophys. J., 95(6):2989-2997, 2008. ISSN 0006-3495.

[164] V. Subramaniam, A.K. Kirsch, and T.M. Jovin. Cell biological applications of scanning near-field optical microscopy (SNOM). Cell. Mol. Biol., 44(5):689-700, 1998.

[165] T. C. Sudhof. The synaptic vesicle cycle. Annu. Rev. Neurosci., 27:509-547, 2004.

[166] T. Suratwala, Z. Gardlund, K. Davidson, and D. R. Uhlmann. Silylated coumarin dyes in sol-gel hosts. 1. structure and environmental factors on fluorescent properties. Chem. Mater., 10:190-198, 1998.

[167] R.B. Sutton, D. Fasshauer, R. Jahn, and A.T. Brunger. Crystal structure of the SNARE complex involved in synaptic exocytosis at 2.4A resolution. Nature, 395: 347-353, 1998. 
[168] S. Takamori, M. Holt, K. Stenius, E. A. Lemke, M. Grønborg, D. Riedel, H. Urlaub, S. Schenck, B. Brügger, P. Ringler, S. A. Müller, B. Rammner, F. Gräter, J. S. Hub, B. L. De Groot, G. Mieskes, Y. Moriyama, J. Klingauf, H. Grubmüller, J. Heuser, F. Wieland, and R. Jahn. Molecular anatomy of a trafficking organelle. Cell, 4: $831,2006$.

[169] K. T. Tokuyasu. A technique for ultracryotomy of cell suspensions and tissues. $J$. Cell. Biol., 57(2):551-65, 1973.

[170] C. K. Ullal, R. Schmidt, S. W. Hell, and A. Egner. Block copolymer nanostructures mapped by far-field optics. Nano Lett., 9(6):2497-2500, 2009.

[171] V.Westphal M.A. LAuterbach S.JAkobs C.A: Wurm C.Eggeling C.Ringemann S.W. Hell V. Belov, K. Kolmakov. Novel hydrophilic and lipophilic rhodamines for labelling and imaging. Eur. Pat. Appl., 2009.

[172] E. F. Vanin, G. I. Goldberg, P. W. Tucker, and O. Smithies. A mouse alpha-globinrelated pseudogene lacking intervening sequences. Nature, 286:222-226, 1980.

[173] A. Vaziri, J. Tang, H. Shroff, and C. V. Shank. Multilayer three-dimensional super resolution imaging of thick biological samples. P. Nat. Acad. Sci. U.S.A., 105(51): 20221-20226, 2008.

[174] X. Wang, X. Ren, K. Kahen, M.A. Hahn, M. Rajeswaran, S. Maccagnano-Zacher, J. Silcox, G.E. Cragg, A.L. Efros, and T.D. Krauss. Non-blinking semiconductor nanocrystals. Nature, 459(7247):686-689, 2009. ISSN 0028-0836.

[175] M. L. Watson. Staining of tissue sections for electron microscopy with heavy metals. J. Cell Biol., 4(6):727-730, 1958.

[176] M. R. Wenk and P. De Camilli. Protein-lipid interactions and phosphoinositide metabolism in membrane traffic: Insights from vesicle recycling in nerve terminals. P. Natl. Acad. Sci. U.S.A., 101(22):8262-8269, 2004.

[177] V. Westphal, M A Lauterbach, A Di Nicola, and S. W. Hell. Dynamic far-field fluorescence nanoscopy. New J. Phys., 9:435, 2007.

[178] J. G. White, E. Southgate, J. N. Thomson, and S. Brenner. The structure of the nervous system of the nematode Caenorhabditis elegans. Philos. T. Roy. Soc. B, 314(1165):1-340, 1986.

[179] M. Wienisch and J. Klingauf. Vesicular proteins exocytosed and subsequently retrieved by compensatory endocytosis are nonidentical. Nat. Neurosci., 9(8):10191027, 2006.

[180] D. Wildanger, E. Rittweger, L. Kastrup, and S. W. Hell. STED microscopy with a supercontinuum laser source. Opt. Express, 16(13):9614-9621, 2008. 
[181] K. I. Willig, S. O. Rizzoli, V. Westphal, R. Jahn, and S. W. Hell. STED-microscopy reveals that synaptotagmin remains clustered after synaptic vesicle exocytosis. $\mathrm{Na}$ ture, 440(7086):935 - 939, 2006.

[182] K. I. Willig, B. Harke, R. Medda, and S. W. Hell. STED microscopy with continuous wave beams. Nat. Methods, 4(11):915-918, 2007.

[183] T. Wilson and A.R. Carlini. Size of the detector in confocal imaging systems. Opt. Lett., 12(4):227, 1987.

[184] T. Wilson and C. J. R. Sheppard. Imaging and superresolution in the harmonic microscope. Opt. Acta, 26:761-770, 1979.

[185] S. Wischnitzer. Introduction to electron microscopy. Pergamon, Oxford, 1981.

[186] H. P. Wohnsiedler. Polymerization in melamine-formaldehyde molded resins. Ind.Eng. Chem., 45(10):2307-2311, 1953. ISSN 0019-7866.

[187] D. Wynforth T., B. Stringer, and G. R. Newman. Hydroxyethylmethacrylate embedding - an improved technique. Med. Lab. Sci., 38(2):121-122, 1981.

[188] Q. Zhang, Y. Li, and R.W. Tsien. The dynamic control of kiss-and-run and vesicular reuse probed with single nanoparticles. Science, 323(5920):1448-53, 2009. 


\section{Thanks.......}

In the end I'd like to thank a number of people, who contributed by some means or other to the outcome of this thesis:

- Prof. Dr. Stefan W. Hell for the possibility to perform the presented work in his department, which was accompanied by outstanding working conditions. His continuous motivation and his pivotal suggestions, together with the conceeded freedom, directed this thesis to the in hand result.

- Prof. Dr. Jürgen Troe for his kindly agreement in acting as the main referee for this thesis, for the uncomplicated handling of administrative tasks and last but not least for his interest in my work.

- Dr. Lars Kastrup for his supervision and for continously supporting my work with various productive incitements and discussions.

- Dr. Silvio Rizzoli for excellent and prosperous collaboration, which was in many cases invaluable and encouraging.

- Peer Hopmann for complementing parts of this work with biological experiments outrunning of my knowledge.

- Shigeki Watanabe for consistently providing me with worm samples and not resigning on the mysteries of sample mailing.

- Juan Carlos Tapia and Bobby Kasthuri for growing the mice, for the preparation of many challenging samples and for the possibility to benefit from the capacity of their ATUM unit.

- All the people who at times bravely left their setups in my hands: Dominik Wildanger, Johanna Bückers, Dr. Ben Harke, Dr. Katrin Willig, Dr. Birka Hein, Lars Meyer, Dr. Volker Westphal, Marcel Lauterbach.

- Dr. Jonas Fölling for the GS-DIM measurements.

- Dr. Ira Lemm for endless beautiful cells, allthough all in vain...

- Dr. Ellen Rothemmel, Rebecca Medda, Tanja Gilat and Dr.Christian Wurm for endless cells, only sometimes in vain.

- Dr. Wiebke Möbius and Dr. Christian Eggeling for inspiring discussions, as well as the open-minded and patient courtesy to my questions. 
- Dr. Marco Roose and Dr. Andreas Schönle for helping out of (nearly) any IT and software mass.

- Gerd for the supply of all the packages which I already estimated as dead.

- Dr. Lars Kastrup, Jay Jethwa, Veronika Müller, Dr. Jan Keller, Dr. Giuseppe Vicidomini, Dr. Martin Andresen, Dr. Andreas Schönle and Dr. Ulf Hensen for proof-reading (parts of ) this manuscript.

- The whole department of NanoBiophotonics for the pleasant working atmosphere in the office, the labs and on the roof - special thanks go to Anja, Sarah and Josi.

- My fellows from the PhD Commitee for all the blast and the opportunity to look beyond my own nose, not only in matters of research.

Thanks to all the special people in and out of Göttingen, who make life worth it and had their stake in the delightful afterglow I will link to the last (almost) four years. And to my parents, Heinz Jürgen and Ulf - for everything! 


\section{Curriculum vitae}

\section{Angaben zur Person}

Nachname(n) / Vorname(n)

Geburtsdatum

Geburtsort

Staatsangehörigkeit

\section{Schulausbildung}

1987-1991

1991-2000

06.2000

\section{Studium}

2000-2002

10.2002

2002-2005

02.2006

$02.2004-07.2004$

$02.2001-07.2001$

seit 02.2006

Soest in Westf.

deutsch

\section{Punge, Annedore}

04. Oktober 1980

Hellweg-Grundschule, Ampen

Conrad-von-Soest Gymnasium, Soest

Abitur am Conrad-von-Soest-Gymnasium, Soest

Grundstudium der Chemie, Bayerische-Julius-Maximilians-Universität, Würzburg

Vordiplom in Chemie

Hauptstudium der Chemie, Bayerische-Julius-Maximilians-Universität, Würzburg

Diplom in Chemie

Forschungspraktikum am Instituto de Estructura de la Materia, CSIC, Madrid, Spanien

Grundstudium der Biologie, Bayerische-Julius-Maximilians-Universität, Würzburg

Dissertation am Max-Planck-Institut für Biophysikalische Chemie, Abteilung NanoBiophotonics, Göttingen 